Aus der Abteilung Humangenetik

(Prof. Dr. med. Dr. h.c. W. Engel)

im Zentrum Hygiene und Humangenetik

der Medizinischen Fakultät der Universität Göttingen

\title{
MicroRNA Expression Profile of Undifferentiated and Differentiating Pluripotent Cells
}

\author{
Inaugural-Dissertation \\ zur Erlangung des Doktorgrades \\ der Medizinischen Fakultät \\ der Georg-August-Universität zu Göttingen
}

vorgelegt von

Angeliki Pantazi

aus Amarousio Attikis, Griechenland 
Dekan:

I.Berichterstatter:

II.Berichterstatter/in:

III.Berichterstatter/in:

Tag der mündlichen Prüfung:
Prof. Dr. med. C. Frömmel

Prof. Dr. med. Dr. h.c. W. Engel 
Contents....................................................................................

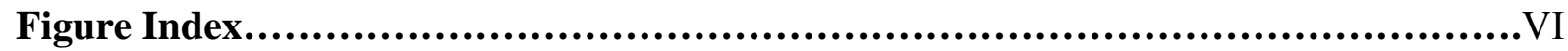

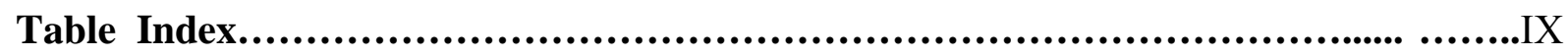

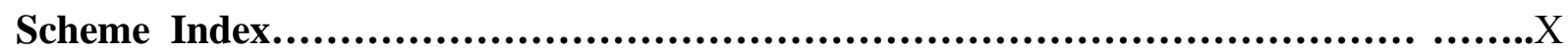

Abbreviations.....................................................................................

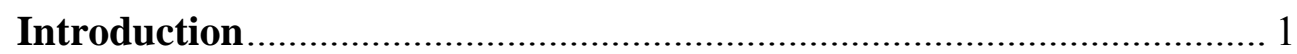

Pluripotent stem cells: definition and criteria ......................................... 1

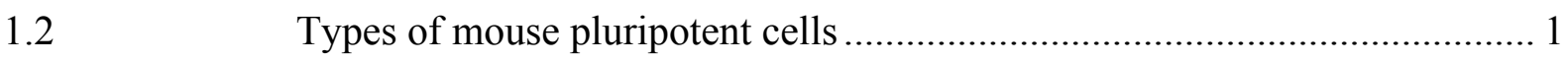

1.2.1 Mouse embryonic stem cells (ESCs) ................................................... 3

1.2.2 Mouse embryonic germ cells (EGCs) ................................................. 4

1.2.3 Mouse multipotent adult germline stem cells (maGSCs) ........................... 5

1.3 Differentiation in vitro of pluripotent stem cells ......................................... 5

1.3.1 Controlling the differentiation of pluripotent stem cells............................. 5

Evaluation of pluripotency and stem cell differentiation............................. 6

miRNAs: biogenesis and function ..................................................... 7

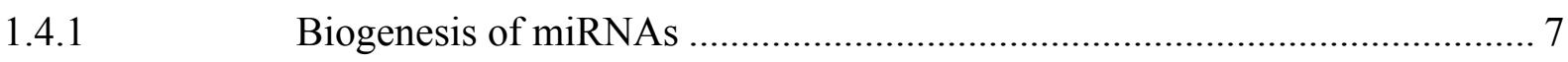

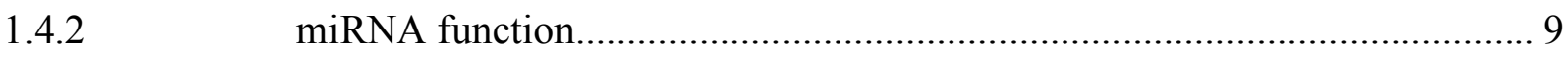

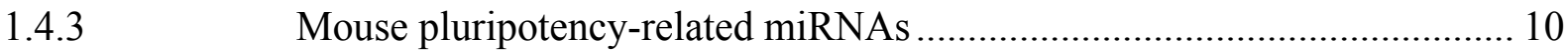

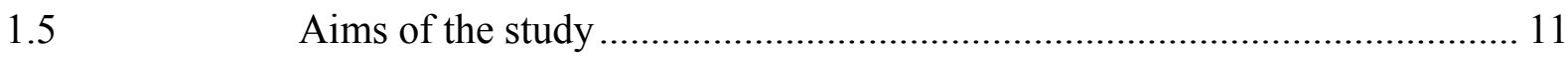

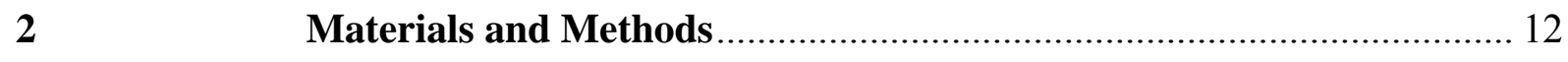

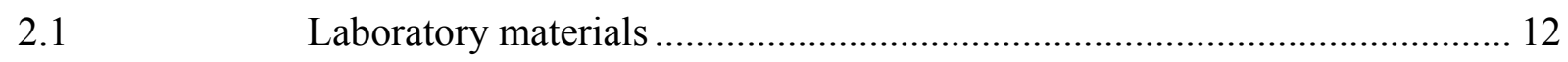

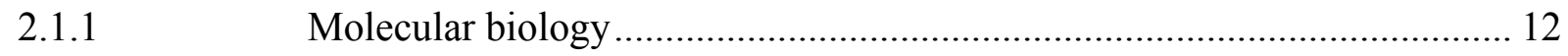

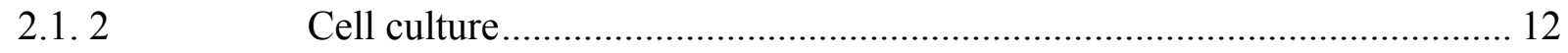

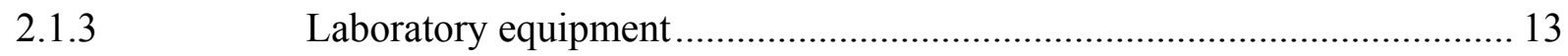

2.1.4 Chemicals, biochemical and biological materials................................... 14

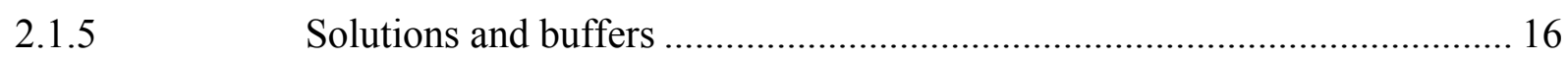

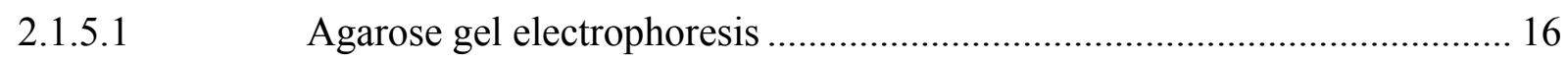




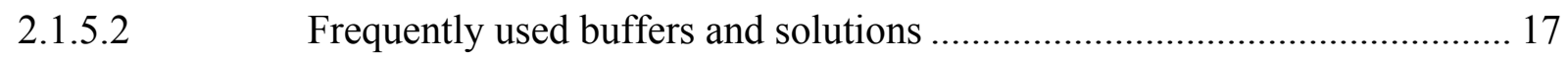

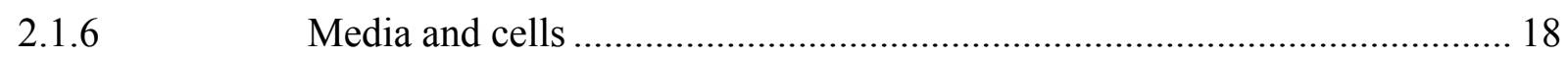

2.1.6.1 Eukaryotic cell culture media and cells ................................................. 18

2.1.6.2 Bacteria culture media and cells .............................................................. 19

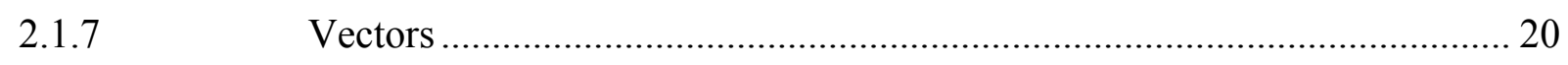

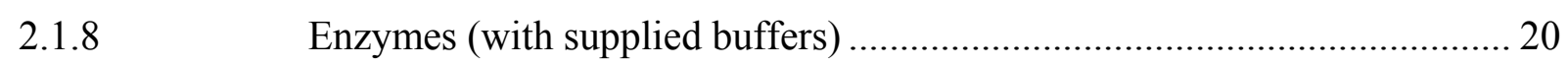

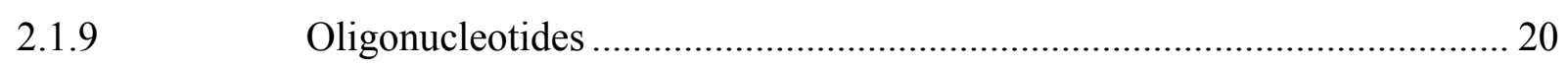

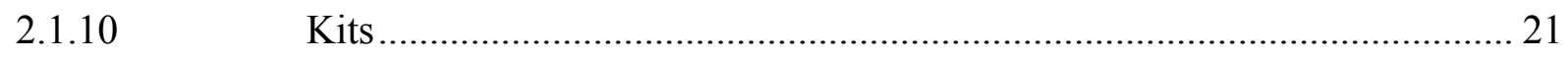

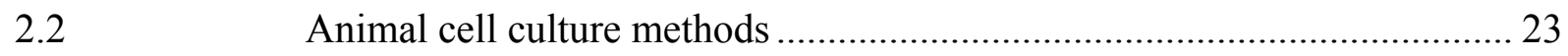

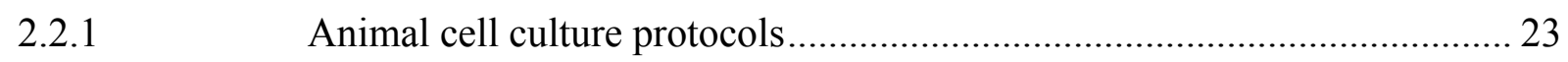

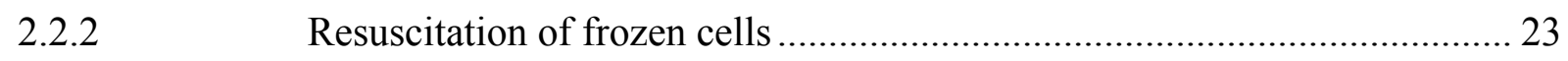

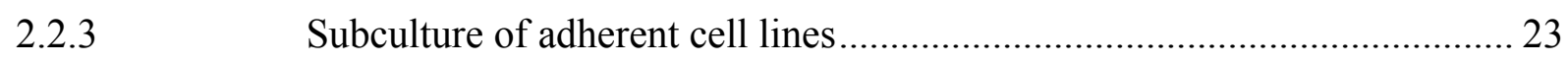

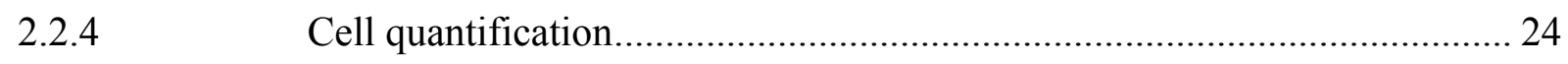

2.2.5 Mouse Embryonic Fibroblasts (MEFs) and feeder layers .......................... 25

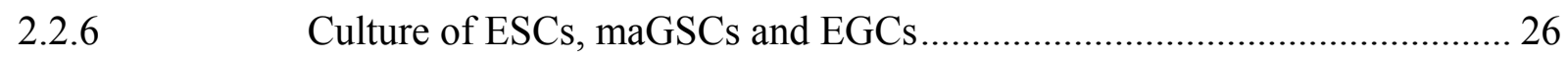

2.2.7 Culture of ESCs, maGSCs and EGCs: differentiation conditions ............... 26

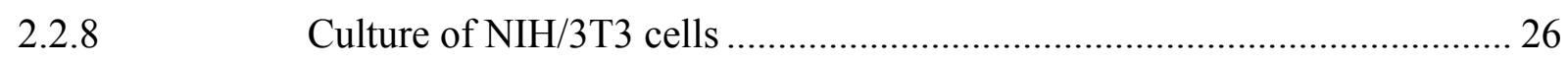

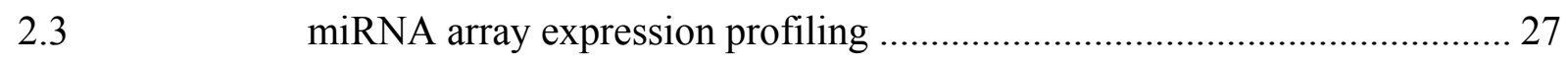

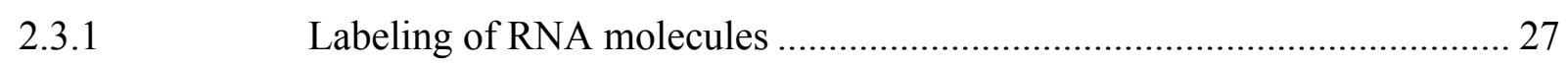

2.3.2 Description of miRCURY'TM LNA microRNA Array slides ...................... 28

2.3.3 Hybridization and washing of the arrays ................................................ 29

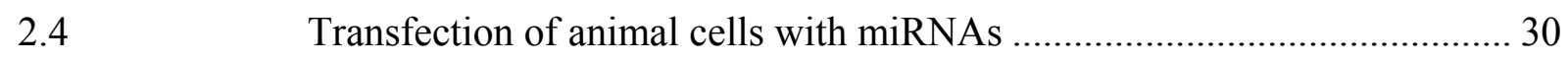

2.4.1 miRNA molecules used in cell transfection ............................................. 30

Optimization of transfection conditions..................................................... 31

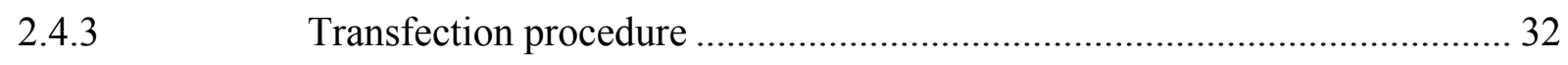

2.5 Co-transfection of animal cells with miRNAs and plasmid DNA.............. 32

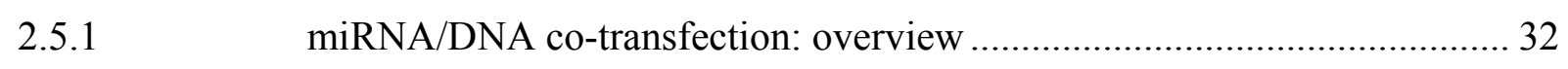

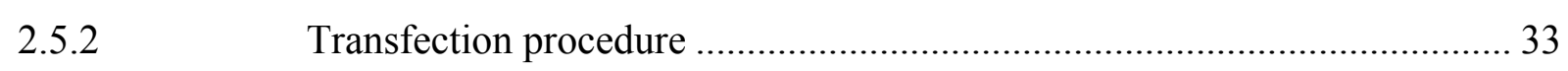

2.6 pMIR-REPORT ${ }^{\mathrm{TM}}$ miRNA Expression Reporter Vector System,

Luciferase assay system and $\beta$-galactosidase assay system ...................... 34

2.6.1 pMIR-REPORT ${ }^{\mathrm{TM}}$ miRNA Expression Reporter Vector System ............... 34

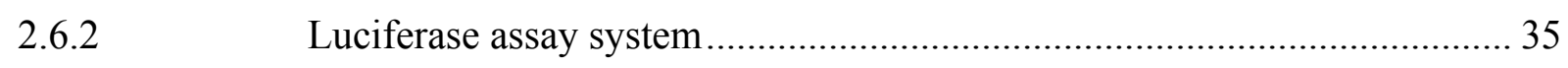

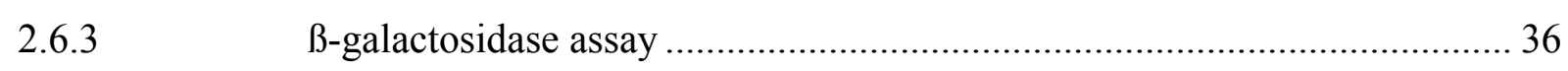

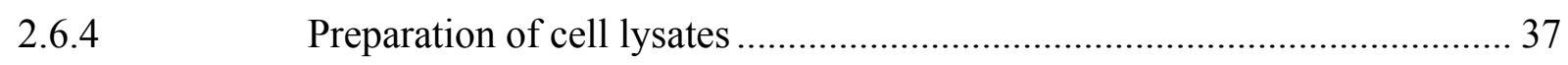


2.6.5 Measurement in plate-reading luminometer with injector ......................... 37

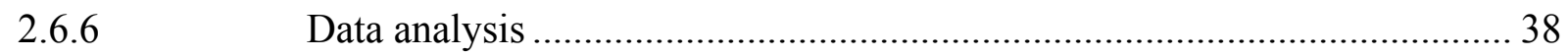

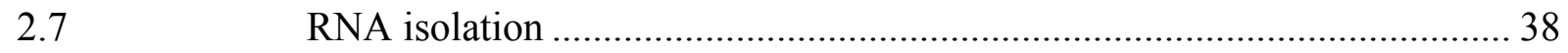

2.7.1 Isolation of total RNA including small RNAs .......................................... 38

2.7.2 Determination of nucleic acid concentration .......................................... 40

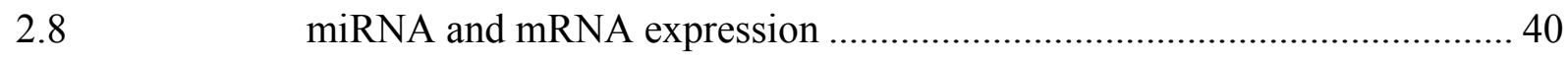

2.8.1 Conversion of miRNAs and other small non-coding RNAs into cDNA (reverse-transcription step) .............................................................. 41

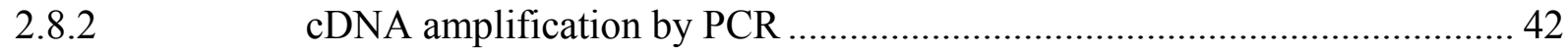

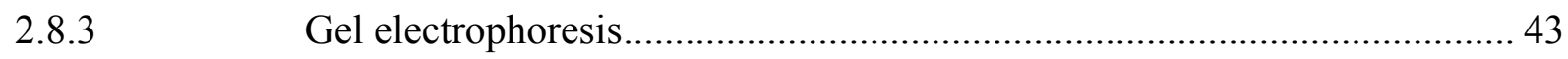

2.8.4 Real-time qRT-PCR for miRNAs (real-time qRT-PCR step) .................... 43

2.8.5 Real-time qRT-PCR for mRNAs (real-time qRT-PCR step) ..................... 46

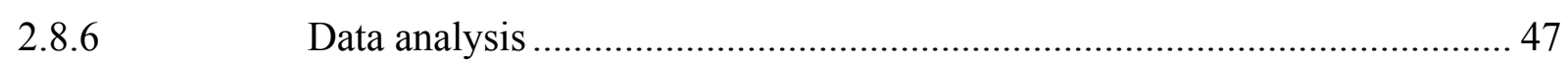

2.9 Methods for subcloning and sequencing of RT-PCR products .................. 48

2.9.1 Extraction of DNA from agarose gel ..................................................... 48

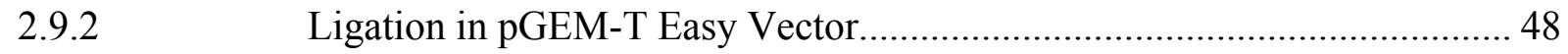

2.9.3 Transformation of DH5a competent bacteria ......................................... 49

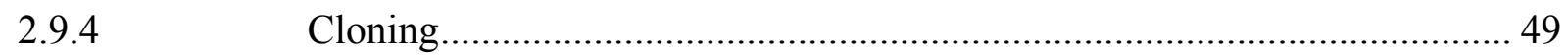

2.9.5 Small-scale isolation of plasmid DNA (Mini preparation) .......................... 50

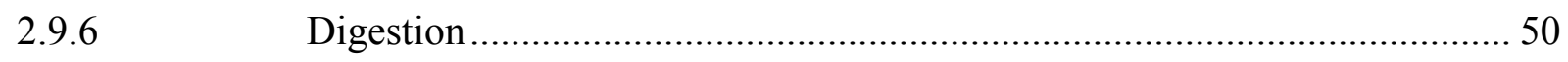

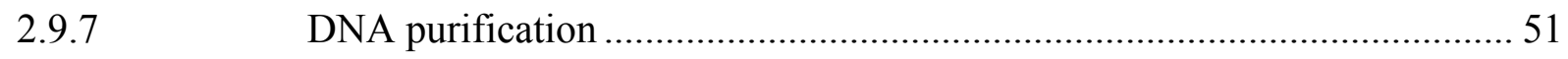

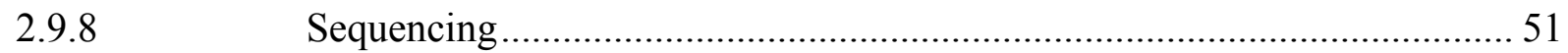

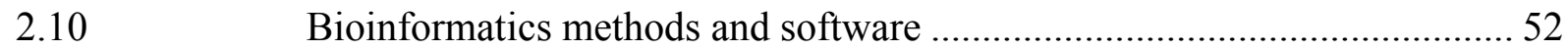

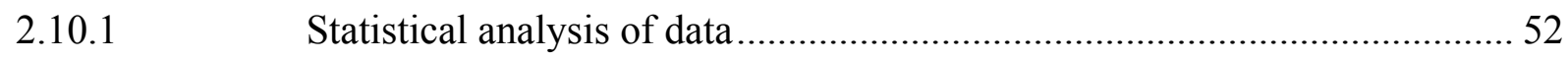

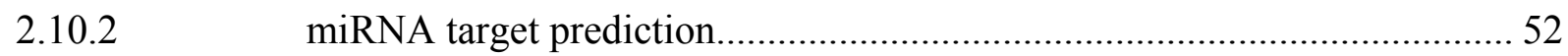

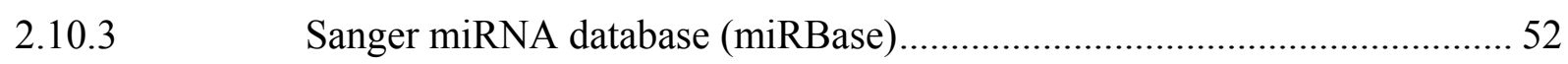

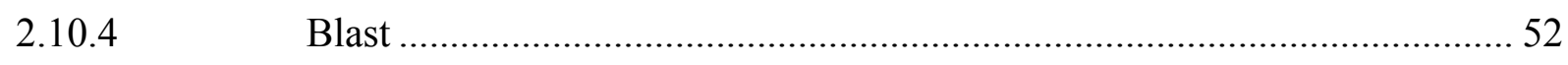

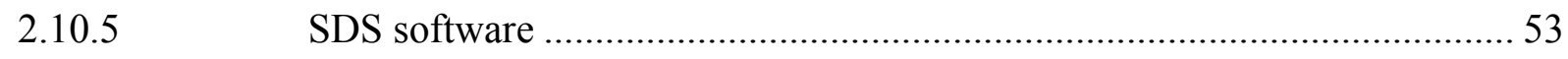

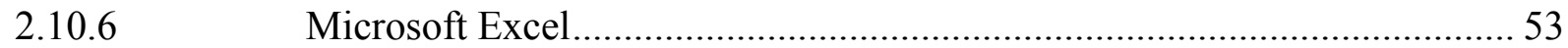

$3 \quad$ Results

3.1 miRNA array expression profiling of ESCs and maGSCs during

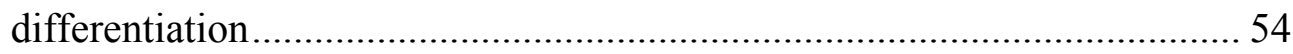


3.1.1 Comparison between undifferentiated ESCs (ESCs_Undiff) and differentiated (ESCs_Diff) after a 21-day treatment under differentiation conditions.

3.1.2 Comparison between undifferentiated maGSCs (maGSCs_Undiff) and differentiated (maGSCs_Diff) after a 21-day treatment under differentiation conditions 59

Expression profiling of members of miR-17-92 cluster during

differentiation in ESCs and maGSCs.....

Overexpression of miR-302 cluster is not sufficient for maintenance of pluripotency

Influence of miR-302 cluster on in vitro differentiation of pluripotent cells

Use of luciferase assay for confirmation of predicted targets of miR-302 cluster. 
4.3 The role of miRNAs in pluripotent stem cells: miR-17-92 cluster.. 115 miR-302 cluster is a key regulator of differentiation in pluripotent stem cells.. 


\section{Figure Index}

Fig. 1:

miRNA biogenesis 8

Fig. 2: $\quad$ Bioluminescent reaction catalyzed by firefly luciferase. 36

Fig. 3: miRNA array expression profiling of ESCs and maGSCs during differentiation 55

Fig. 4: $\quad$ Experimental layout of miRNA array expression profiling of ESCs and maGSCs during differentiation . 56

Fig. 5: Validation of miR-290 cluster by real-time qRT-PCR 61

Fig. 6: Validation of miR-17 and miR-20a by real-time qRT-PCR. 63

Fig. 7:

Validation of miR-720 by real-time qRT-PCR. 64

Fig. 8:

Validation of miR-320 by real-time qRT-PCR. 66

Fig. 9:

Differentiation of ESCs and maGSCs 68

Fig. 10:

miR-17 during differentiation of ESCs and maGSCs 69

Fig. 11: miR-19b during differentiation of ESCs and maGSCs 70

Fig. 12: miR-20a during differentiation of ESCs and maGSCs .71

Fig. 13: miR-20b during differentiation of ESCs and maGSCs .73

Fig. 14: Differentiation of EGCs . .75

Fig. 15: miR-290, miR-302a and miR-17 during differentiation of EGCs 76

Fig. 16:

Overexpression of miR-302 cluster in ESCs, maGSCs and EGCs 78

Fig. 17:

Optimization of transfection conditions in ESCs. 80

Fig. 18: miR-302a (A), miR-302b (B) and miR-302d (C) in differentiating ESCs by overexpression of miR-302 cluster

Fig. 19: $\quad$ Oct4 in differentiating ESCs (A), maGSCs (B) and EGCs (C) by overexpression of miR-302 cluster

Fig. 20: $\quad$ Brachyury in differentiating ESCs (A), maGSCs (B) and EGCs (C) by overexpression of miR-302 cluster 86

Fig. 21: $\quad$ Eomesodermin and Fibroblast Growth Factor 8 (Fgf-8) in differentiating ESCs (A), maGSCs (B) and EGCs (C) by overexpression of miR-302 cluster 88

Fig. 22: $\quad$ Nestin in differentiating ESCs (A), maGSCs (B) and EGCs (C) by overexpression of miR-302 cluster 90

Fig. 23: $\quad$ Dppa3 in differentiating ESCs (A), maGSCs (B) and EGCs (C) by overexpression of miR-302 cluster 91 
Fig. 24: $\quad$ Predicted miRNAs that target the mRNAs of Brachyury (A) and Dppa3 (B) according to TargetScan/Release 4.2 _................................... 93

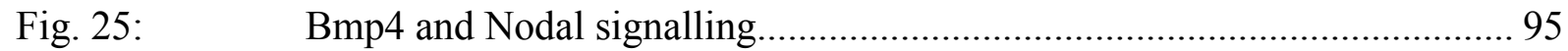

Fig. 26: Validation of generated miRNA Luciferase Vectors for Smad2 (CMV-LUCSmad2 3' UTR) (A) and Dkk1 (CMV-LUC-Dkk1 3' UTR) (B)............... 101

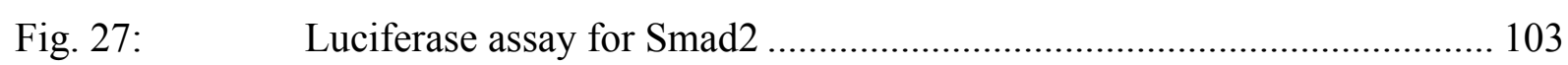

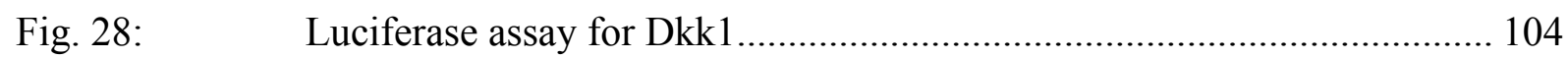

Fig. 29: $\quad$ Expression of miR-290 cluster in ESCs and maGSCs during

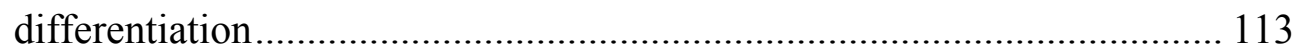

Fig. 30: $\quad$ Expression of miR-302 cluster in ESCs and maGSCs during

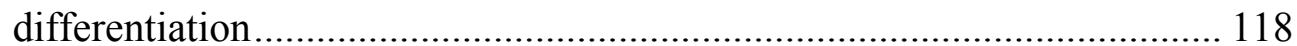




\section{Table Index}

Tab. 1: $\quad$ Differentially expressed miRNAs between undifferentiated ESCs

(ESCs_Undiff) and differentiated ESCs (ESCs_Diff)....

Tab. 2: $\quad$ Differentially expressed miRNAs between undifferentiated maGSCs

(maGSCs_Undiff) and differentiated maGSCs (maGSCs_Diff).....

Tab. 3: $\quad$ Predicted targets of miR-302 cluster among activators + /inhibitors of

Bmp4, Nodal and Wnt pathways according to TargetScan/Release 4.2..... 97 


\section{Scheme Index}

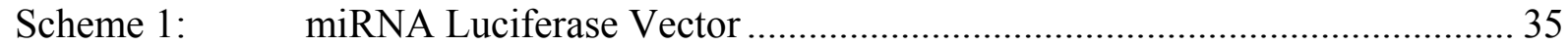

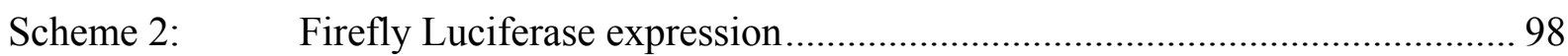

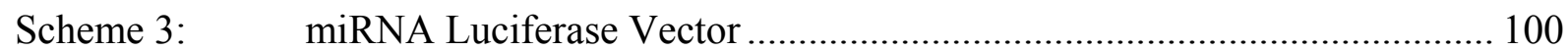




\section{Abbreviations}

A

AD

Ago

ATP

B

ß-gal

BLAST

bp

BSA

$\mathrm{C}$

${ }^{\circ} \mathrm{C}$

cDNA

C.elegans

CIP

$\mathrm{cm}$

CMV

$\mathrm{Cy}-$

dATP

dCTP

$\mathrm{ddH}_{2} \mathrm{O}$

DEPC

dGTP

diff

DMEM

DMSO

DNA

DNase
Purine base adenine

Alzheimer's disease

Argonaute

Adenosine triphosphate

Beta

B-galactosidase

Basic Local Alignment Search Tool

Base pairs

Bovine serum albumin

Pyrimidine base cytosine

Grades Celsius

complementary DNA

Caenorhabditis elegans

Calf Intestinal Phosphatase

Centimetre

Cytomegalovirus

cyanine

Deoxyadenosine triphosphate

Deoxycytidine triphosphate

Distilled water

Diethylpyrocarbonate

Deoxyguanosine triphosphate

differentiated

Dulbecco's Modified Eagle Medium

Dimethylsulfoxid

Deoxyribonucleic acid

Deoxyribonuclease 


\begin{tabular}{|c|c|}
\hline dNTP & Deoxynucleoside triphosphate \\
\hline dsRNA & double-stranded RNA \\
\hline $\mathrm{dT}$ & Desoxythymidin \\
\hline DTT & Dithiotreitol \\
\hline dTTP & Deoxythymidine triphosphate \\
\hline $\mathrm{ECC}(\mathrm{s})$ & Embryonic carcinoma cell(s) \\
\hline E.coli & Escherichia coli \\
\hline EDTA & Ethylendiaminetetraacetic acid \\
\hline EBs & Embryoid bodies \\
\hline $\mathrm{EGC}(\mathrm{s})$ & Embryonic germ cell(s) \\
\hline EpiSC(s) & Epiblast stem cell(s) \\
\hline $\mathrm{ESC}(\mathrm{s}), \mathrm{ES}$ & Embryonic stem cell(s) \\
\hline $\mathrm{EtOH}$ & Ethanol \\
\hline et al. & et alii (and others) \\
\hline FACS & Fluorescent-activated cell sorting \\
\hline $\mathrm{FB}(\mathrm{s})$ & Fibroblast(s) \\
\hline FCS & Foetal bovine serum \\
\hline Fig. & figure \\
\hline $\mathrm{FL}(\mathrm{s})$ & Feeder layer(s) \\
\hline G & Purine base Guanine \\
\hline g & Gram \\
\hline Gel & gelatine \\
\hline h, hrs & hour(s) \\
\hline HEPES & 4-(2-hydroxyethyl)-1-piperazineethanesulfonic acid \\
\hline ICM & Inner cell mass \\
\hline $\operatorname{Id}(\mathrm{s})$ & Inhibitor of differentiation proteins \\
\hline IL- & Interleukin- \\
\hline iPS cell(s) & Induced pluripotent stem cells \\
\hline IPTG & Isopropyl-ß-D-1-thiogalactopyranoside \\
\hline
\end{tabular}


$\mathrm{kb}$

$\mathrm{kDa}$

1

LIF

LNA

LUC

$\mathrm{M}$

m

$\mu$

maGSC(s)

MCS

MEF(s)

$\min$

miRNA, miR-

$\mathrm{mM}$

MOPS

mRNA

$\mathrm{n}$

NCBI

Neg

nt

ORF

$\mathrm{P}$

PBS

PCR

PFA

PGC(s)

PMSF

pos
Kilo base pairs

Kilodalton

Liter

Leukemia Inhibitory Factor

Locked Nucleic Acid

luciferase

Molarity

Milli

Micro

Multipotent adult germline stem cell(s)

multiple cloning site

Mouse embryonic fibroblast(s)

minute(s)

MicroRNA

Millimolar

3-(N-morpholino)-propanesulfonic acid

Messenger-RNA

Nano

National Center for Biotechnology Information

negative

Nucleotide

open reading frame

Pico

Phosphate Buffered Saline

Polymerase Chain Reaction

Paraformaldehyde

Primordial germ cell(s)

Phenylmethanesulfonilfluoride

positive 
$\mathrm{PP}_{\mathrm{i}}$

pre-miRNA

pri-miRNA

qRT-PCR

RA

RISC

RLB

RNA

RNase

rpm

RT-PCR

SDS

$\mathrm{sec}$

$\mathrm{SF}$

S.O.C.

$\mathrm{T}$

$\mathrm{Tm}$

Tris

U

undiff

UTR(s)

Vol.

$\mathrm{v} / \mathrm{v}$

X-Gal pyrophosphate

precursor-miRNA

primary miRNA

quantitative Reverse-Transcription-PCR

retinoic acid

RNA Induced Silencing Complex

Reporter Lysis Buffer

Ribonucleic acid

Ribonuclease

rounds per minute

Reverse-Transcription-PCR

Sodium dodecyl sulfate

second(s)

Steel Factor

Super Optimal broth with Catabolite repression

Pyrimidine base thymidine

melting temperature

Tris(hydroxymethyl)-aminomethane

Unit

undifferentiated

,untranslated region(s) “

Volume

Volume/Volume

5-brom-4-chlor-3-indolyl- $\beta$-D-galactopyranoside 


\section{Introduction}

\subsection{Pluripotent stem cells: definition and criteria}

Pluripotent stem cells can proliferate continuously and are able to differentiate into all cells of an organism (apart from extra-embryonic tissues) and give rise to cells of all three germ layers (endoderm, mesoderm and ectoderm) (Schöler 2007). Apart from these two key features pluripotent cells also fulfill additional criterias such as:

- Contribution to various somatic cell types upon injection of the cells into mouse blastocysts and formation of chimeric mice.

- Formation of teratomas after injection of the cells into extrauterine sites of immunodeficient mice.

- Maintenance of normal karyotype.

- Generation of clonally derived cell cultures, originating from a single cell that can give rise to a whole cell line consisting of identical cells

- Expression of transcription factors, such as Oct4, Nanog and Sox2 (Niwa 2007; Buitrago and Roop 2007; Yu and Thomson 2008).

\subsection{Types of mouse pluripotent cells}

Nowadays, there is a variety of mouse pluripotent cell lines cultured under established culture conditions, which share the two fundamental properties of pluripotency, prolonged proliferation in vitro and multilineage differentiation. These cell lines arise from: a. ex vivo cultures of isolated early embryonic cells and cells of the germ lineage or $b$. reprogrammed somatic cells that have 
acquired pluripotency-related character (Lengner and Jaenisch 2008). In detail, the following types of pluripotent cell lines are available:

\section{a. Pluripotent cell lines isolated from early embryos or cells of the germ} lineage:

- Embryonal carcinoma cells (ECCs). ECCs were the first pluripotent stem cells to be isolated and stably propagated in vitro. They are derived from malignant germ cell tumours called teratocarcinomas (Kleinsmith and Pierce 1964; Kahan and Ephrussi 1970).

- Embryonic stem cells (ESCs). ESCs are derived from the inner cell mass (ICM) of the mouse blastocyst (Evans and Kaufman 1981; Martin 1981), however, isolation of ESCs from individual blastomeres of two- to eightcell stage embryos has also recently been described (Chung et al. 2006; Wakayama et al. 2007). Other pluripotent cells that have been recently established are the epiblast stem cells (EpiSCs), which have been derived from epiblasts isolated from E5.5 to E6.5 post-implantation mouse embryos (Brons et al. 2007) (see also 1.2.1).

- Embryonic germ cells (EGCs). EGCs are derived from primordial germ cells (PGCs), which are precursors of the germ cell line in mouse embryo (Matsui et al. 1992; Resnick et al. 1992) (see also 1.2.2).

- Multipotent adult germline stem cells (maGSCs). maGSCs are derived from both neonatal and adult mouse testes (Kanatsu-Shinohara et al. 2004; Guan et al. 2006) (see also 1.2.3). 


\section{b. Pluripotent cell lines derived from reprogrammed somatic cells that have acquired pluripotency-related character:}

- Pluripotent hybrid cells, which result from the fusion of a somatic cell nucleus with ESCs, ECCs or EGCs (Serov et al. 2001; Ambrosi et al. 2007; Han DW et al. 2008).

- Induced pluripotent stem cells (iPS cells). iPS cells are somatic cells reprogrammed to the pluripotent status by the overexpression of pluripotency-related transcription factors (Takahashi and Yamanaka 2006; Wernig et al. 2007).

In 1.2.1, 1.2.2 and 1.2.3 the mouse pluripotent cell lines that were used in this study (ESCs, maGSCs and EGCs) are described in detail.

\subsubsection{Mouse embryonic stem cells (ESCs)}

The observation that early mouse embryos, which were injected into extrauterine sites, could give rise to teratocarcinomas demonstrated that a population of cells with a high pluripotent potential is present in early embryos (Stevens 1970). Such experiments led to the isolation of the first mouse ESCs from blastocysts. These cells are able to differentiate into all three germ layers and form teratomas when injected into mice. Moreover, ESCs contribute widely to chimeras and maintain a normal karyotype (Evans and Kaufman 1981; Martin 1981).

In order to prevent their differentiation, ESCs are cultured under specific culture conditions including mitotically inactivated mouse embryonic fibroblast feeder 
layers (MEFs) and serum-containing medium in the presence of Leukemia Inhibitory Factor (LIF). LIF is a member of the IL-6 cytokine family that stimulates self-renewal of mouse ESCs through the activation of STAT3. One of the key targets of LIF-STAT3 signalling is the transcription factor c-Myc, which plays an important role in cell proliferation. Wnt signalling pathway has a synergistic effect with LIF-STAT3, as it upregulates both STAT3 and c-Myc (Liu et al. 2007). However, LIF-STAT3 signalling is necessary but not sufficient for maintenance of pluripotency. In serum-containing culture medium other factors, like Bmp4, are present and contribute to ESC self-renewal (Ying et al. 2003). Bmp4 is a member of the TGFß superfamily and acts via activation of Smad1/5/8 pathway (Liu et al. 2007) by inducing the expression of Ids (inhibitor of differentiation proteins) and by blocking neural differentiation and other pathways that promote differentiation (Liu et al. 2007).

\subsubsection{Mouse embryonic germ cells (EGCs)}

Embryonic germ cells (EGCs) are derived from primordial germ cells (PGCs). Their initial derivation requires a combination of feeder layer, LIF, stem cell factor (Steel factor, SF) and fibroblast growth factor (FGF). Under these culture conditions PGCs continue to proliferate and form EGC lines (Matsui et al. 1992; Resnick et al. 1992). EGCs are similar in morphology with mouse ESCs, express typical ESC markers, such as Oct4 and SSEA1 and contribute extensively to chimeric mice (Matsui et al. 1992). 


\subsubsection{Mouse multipotent adult germline stem cells (maGSCs)}

Mouse multipotent adult germline stem cells (maGSCs) were successfully derived from testes of adult mice by the group of Prof. Engel in Göttingen (Guan et al. 2006). These ES-like cells are phenotypically similar to ESCs and EGCs. They differentiate into various types of somatic cells in vitro and produce teratomas after inoculation into immunodeficient mice. Furthermore, maGSCs are able to contribute to chimeras when injected into blastocysts (Guan et al. 2006). These results were confirmed by other groups (Seandel et al. 2007; Izadyar et al. 2008). It has to be noted that multipotent germline stem cells have been also previously isolated from neonatal mouse testes (Kanatsu-Shinohara et al. 2004).

\subsection{Differentiation in vitro of pluripotent stem cells}

\subsubsection{Controlling the differentiation of pluripotent stem cells}

Pluripotent stem cells, by definition, renew and can also differentiate. Under conventional culture conditions using serum-containing culture media and LIF, pluripotent cells maintain a sensitive balance between self-renewal and differentiation; in the absence of LIF and in the presence of serum, differentiation dominates over self-renewal (Wiles and Proetzel 2006).

In vitro differentiation of mouse ESCs is often performed through the formation of aggregated clumps of cells known as embryoid bodies (EBs). These structures contain intermediate progenitor cells for the three germ layers. However, although EB-mediated induction of differentiation of ESCs is simple to perform, the efficiency of this method and the ability to reproduce the results are limited. 
The nature of obtained cells is difficult to regulate or predict (Wiles and Proetzel 2006).

It has been demonstrated that mouse ESCs can be efficiently differentiated into mesoderm in serum-containing media (Keller et al. 1993). The finding that the effect of serum is so dominant in ESCs during in vitro differentiation led to the idea of chemically defined media in which serum was replaced by chemically defined reagents. Using this approach it could be demonstrated that mesoderm is induced by the TGF- $\beta$ superfamily members, bone morphogenetic protein- $2,-4$, -7 (Bmp2, Bmp4, Bmp7) or activin A. In the absence of mesoderm-inducing factors, neuroectoderm development occurs (Wiles and Johansson 1999). Conventionally, neural differentiation has been induced by formation of embryoid bodies in the presence of retinoic acid (RA), a strong inducer of differentiation (Rolletschek et al. 2002). The use of defined culture systems can contribute to both understanding and directing the differentiation of mouse ESCs into desired cell types (Wiles and Proetzel 2006).

\subsubsection{Evaluation of pluripotency and stem cell differentiation}

There are several methods for the evaluation of stem cell differentiation, starting from the morphological assessment of the in vitro differentiation of embryoid bodies (Wiles and Proetzel 2006). In addition, the efficiency and direction of differentiation can be estimated by detection of pluripotent and lineage marker genes. Genes such as Oct4, Nanog and Sox 2 are widely used to confirm the pluripotent status of cells (Niwa 2007; Buitrago and Roop 2007); marker genes for mesoderm are Brachyury, Fgf8 and Eomesodermin (Keller 2005; Alisch et al. 2007); markers for endoderm are Gata4 and Hnf4 (Sladek et al. 1990; Rubin 
2007); markers for ectoderm are Nestin and Pax6 (Lendahl et al. 1990; Duparc et al. 2006).

In order to monitor the status of differentiated cells the above marker genes can be used in several methods, such as RT-PCR, quantitative RT-PCR, in situ hybridization, immunocytochemistry and Western blotting. Finally, microarray analysis is also an informative tool for obtaining a global view of changes on mRNA level due to cell differentiation (Wiles and Proetzel 2006).

\section{4 miRNAs: biogenesis and function}

\subsubsection{Biogenesis of miRNAs}

MiRNAs are a recently identified class of cellular single-stranded RNAs of 1925 nucleotides in length. miRNA biogenesis includes miRNA transcription in the nucleus, the export of miRNAs from the nucleus to the cytoplasm, and subsequent processing and maturation in the cytoplasm (Singh et al. 2008) (Fig. $1)$. 


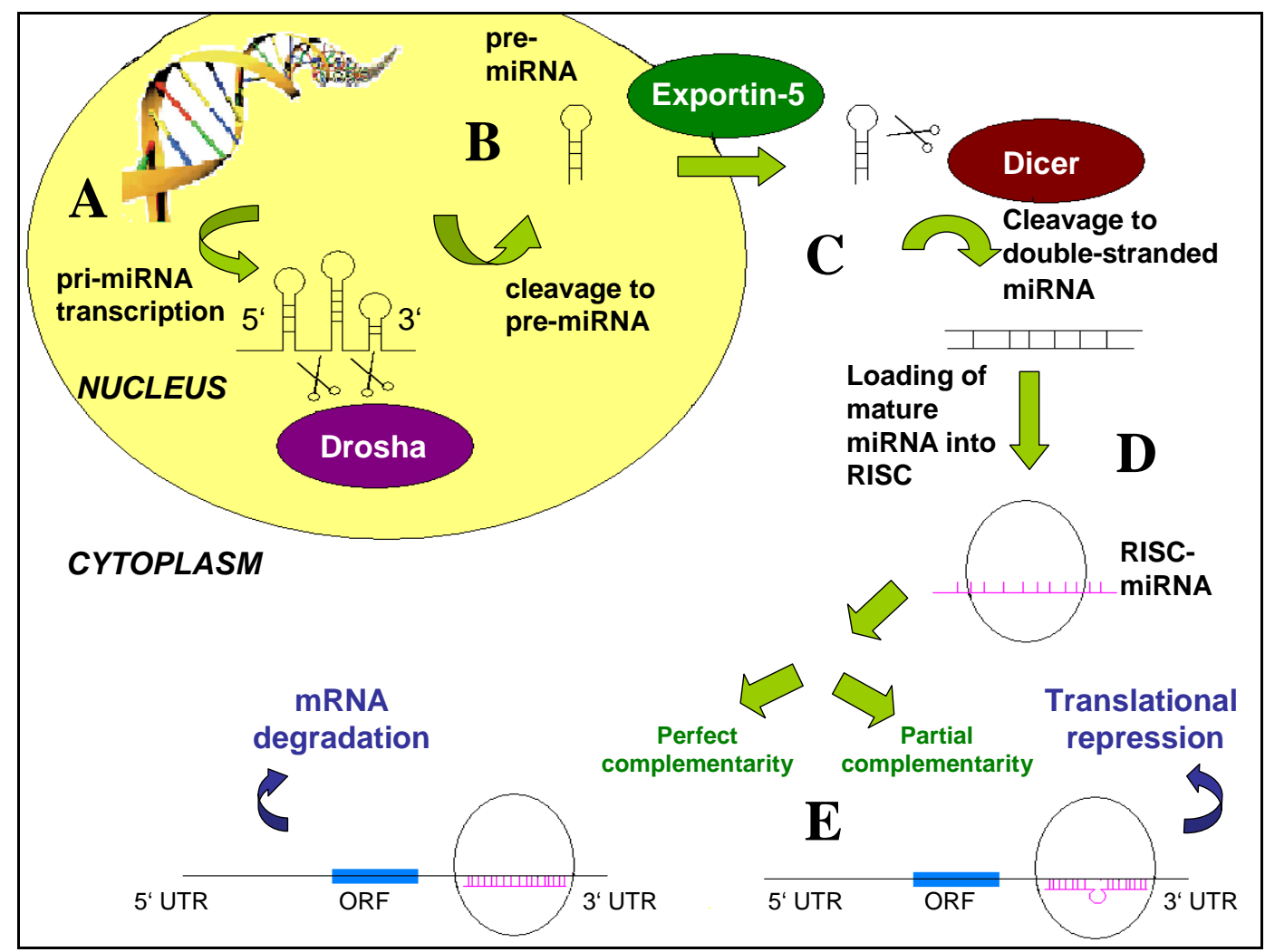

Figure 1. miRNA biogenesis. A: miRNAs are expressed in the nucleus as parts of long primary miRNA transcripts (pri-miRNA) that have 5 caps and 3 poly(A) tails. B: The hairpin structure that pri-miRNA forms acts as a signal for digestion by a ribonuclease (Drosha) to produce the precursor miRNA (premiRNA). C: Exportin-5 mediates nuclear export of pre-miRNAs. D: A cytoplasmic dsRNA nuclease (Dicer) cleaves the pre-miRNA. The singlestranded mature miRNA associates with a complex that is similar, if not identical, to the RNA Induced Silencing Complex (RISC). E: The miRNA/RISC complex leads to protein degradation or represses protein translation by binding to sequences in the $3^{\prime}$ untranslated region of specific mRNAs.

In most cases, the transcription of miRNA genes is mediated by RNA polymerase II, resulting in long primary miRNA (pri-miRNA) transcripts. The sequence of the miRNA remains embedded in the arms of the stem loop. The 
pri-miRNA is processed by a ribonuclease called Drosha into a hairpin RNA molecule of about 65 nucleotides, called precursor miRNA (pre-miRNA) (Singh et al. 2008). The Drosha-Dgcr8 complex, also known as microprocessor, is essential for miRNA maturation. Drosha functions as the catalytic subunit, while Dgcr8 (also known as Pasha) recognizes the RNA substrate (Han J et al. 2009). The pre-miRNA is then exported to the cytoplasm by Exportin 5 and it is finally cleaved by a cytoplasmic dsRNA nuclease called Dicer into a small dsRNA duplex, which finally results into the mature 19- to 25-nucleotide miRNA (Singh et al. 2008; Bartel and Chen 2004).

Approximately half of all miRNAs are transcribed from a single polycistronic pri-miRNA (miRNA clusters), indicating that these miRNAs are co-ordinately regulated during development (Bartel 2004; Bartel and Chen 2004).

\subsection{2 miRNA function}

miRNAs mediate gene silencing through different mechanisms at the posttranscriptional level. The fate of the target mRNA when a miRNA binds to its 3' UTR depends on the degree of complementarity between miRNA and target mRNA (Kim 2005). If the complementarity between miRNA and target mRNA is almost perfect, the target mRNA is cleaved. If the complementarity is lower, the interaction results in translational repression. In either case, the highest level of complementarity is found at positions 2-7 nt relative to the $5^{\prime}$ end of miRNA and basepairing at this region appears to be important for target recognition (Kim 2005) (Fig. 1E). 


\subsubsection{Mouse pluripotency-related miRNAs}

Two clusters of miRNAs, miR-290 and miR-302 clusters, are present in various mouse pluripotent stem cells and seem to play a role concerning their pluripotent status.

miR-290 cluster consists of six members (miR-290, miR-291, miR-292, miR293, miR-294, miR-295; Houbaviy et al. 2003) and miR-302 cluster of four members (miR-302a, miR-302b, miR-302c, miR-302d; Strauss et al. 2006). Both clusters were described as mouse ESC-specific, as they are highly expressed in ESCs but downregulated during ESC differentiation and undetectable in adult mouse organs. maGSCs also express in high levels both miR-290 and miR-302 clusters (Zovoilis et al. 2008).

The role of miR-290 and miR-302 clusters in maintenance of pluripotency and in process of differentiation is still not sufficiently defined; however, there is strong evidence that these two miRNA clusters influence both proliferation and differentiation of various pluripotent cells (Kanellopoulou et al. 2005; Sinkkonen et al. 2008; Hayashi et al. 2008; Zovoilis et al. 2008). 


\subsection{Aims of the study}

miRNA expression profiles of miR-290 and miR-302 clusters in pluripotent cell lines such as ESCs and maGSCs during differentiation have already been described (Zovoilis et al. 2008). Aim of the present study was:

- To screen all known mouse miRNAs that are downregulated during differentiation in ESCs and maGSCs in vitro (miRNA array profiling).

- To extend the previous study of Zovoilis et al. (2008) to an additional pluripotent cell line, embryonic germ cells (EGCs).

- To examine closely the role of miR-302 cluster in ESCs, maGSCs and EGCs by ectopic overexpression under differentiation conditions. 


\section{Materials and Methods}

\subsection{Laboratory materials}

\subsubsection{Molecular biology}

14 ml-centrifuge tubes

Disposable filter Minisart NMI

Falcon tubes

Filter tips

Glasware

Microcentrifuge tubes

Microliterpipettes

PCR-tubes

Petri plates

Reaction tubes

Reaction tubes

Real-Time PCR plates

Tips without filter
Corning, New York, USA

Sartorius, Göttingen

Schleicher and Schüll, Dassel

Biozym, Hessisch Oldendorf

Schott, Mainz

Eppendorf, Hamburg

Pipetman Gilson Abimed,

Langenfeld

Molecular BioProducts, San Diego, USA

Greiner, Nürtingen

Eppendorf, Hamburg

(RNase-free) Brand, Wertheim

Applied Biosystems, Darmstadt

Eppendorf, Hamburg

\subsubsection{Cell culture}

Cell culture flasks and plates

Culture slides BD
Greiner, Nürtingen or Nunc, Wiesbaden

Falcon, Heidelberg 
Cell culture pipettes

Cryocups

Filtering unit

Pasteur pipettes

6 or $10 \mathrm{~cm}$ Plates

Steril Filters

13 ml-centrifuge plastic tubes

14 ml- centrifuge plastic tubes
Sarstedt, Nümbrecht

Greiner, Nürtingen or Sarstedt,

Nümbrecht

Nalgene, Rochester, USA

Brand, Wertheim

Nunc, Wiesbaden

Sartorius, Göttingen

Sarstedt, Nümbrecht

Corning, New York, USA or

Greiner, Nürtingen

Brand, Wertheim

Webeco, Bad Schwartau

Beckman, München

Beckman, München

Eppendorf, Hamburg

Heraeus, Hanau

Heraeus, Hanau

DNA Sequencer Modell Megabace 1000

Eppendorf Centrifuge 5415 D

Eppendorf Centrifuge 5417 R

Fluorescence microscope BX60

Freezer VIPseries $-86^{\circ} \mathrm{C}$

GeneAmp PCR System 9600

HT7900 Sequence Detection System
Amersham, Freiburg

Eppendorf, Hamburg

Eppendorf, Hamburg

Olympus, Planegg

Sanyo, Tokyo, Japan

Perkin Elmer, Berlin

Applied Biosystems, Darmstadt 
Incubator IR Autoflow Incusafe

Inverted microscope IX81

Light microscope

Microwave oven

MWG Primus 96plus Thermocycler

Neubauer cell chamber

Orion II-Microplate Luminometer

Pipettes

Power supply

PTC-100 Peltier Thermal Cycler

Spectrophotometer Ultraspec 3000

SpeedVac concentrator SVC $100 \mathrm{H}$

Sterilbank HERAsafe

Thermomixer 5436

Ultra low remperature

Freezer $-152^{\circ} \mathrm{C}$

UV-Transilluminator
Sanyo, Tokyo, Japan

Olympus, Planegg

Zeiss, Göttingen

Phillips, Hamburg

MWG Biotech, Ebersberg

Schütt Labortechnik, Göttingen

Berthold Detection Systems,

Pforzheim

Eppendorf, Hamburg

Gibco BRL, Karlsruhe

Bio-Rad, München

Amersham, Freiburg

Schütt, Göttingen

Heraeus, Hanau

Eppendorf, Hamburg

Sanyo, Tokyo, Japan

Herolab, Wiesloch

\subsubsection{Chemicals, biochemical and biological materials}

$1 \mathrm{~kb}$ DNA ladder

Agar

Agarose

Ampicillin

Ampuwa

$\beta$-Mercaptoethanol

Boric acid
Invitrogen, Karlsruhe

Difco, Detroit, USA

Invitrogen, Karlsruhe

Sigma, Deisenhofen

Fresenius, Bad Homburg

Gibco BRL, Eggenstein

Sigma, Deisenhofen 
Chloroform

Diethyl pyrocarbonate (DEPC)

Dimethylformamid

Dimethylsulfoxid (DMSO)

Dithiotreitol (DTT)

DMEM

dNTPs

Dye Terminator Mix

EDTA

Ethanol

Ethidium bromide

FCS

Ficoll

Gelatin

Glycerol

$\mathrm{HCl}$

HEPES

IPTG

Isopropanol

$\mathrm{KCl}$

L-Glutamine

LIF

Mitomycin C

$\mathrm{NaCl}$

$\mathrm{NaOH}$

Non-essential amino acids

Orange G

Paraformaldehyde

PBS
Baker, Deventer, NL

Sigma, Deisenhofen

Sigma, Deisenhofen

Sigma, Deisenhofen

Applichem, Darmstadt

PAN, Aidenbach

Invitrogen, Karlsruhe

Applied Biosystems

Biomedicals, Eschwege

Baker, Deventer, NL

Sigma, Deisenhofen

PAN, Aidenbach

Applichem, Darmstadt

Sigma, Deisenhofen

Invitrogen, Karlsruhe

Merck, Darmstadt

Merck, Darmstadt

Biomol, Hamburg

Merck, Darmstadt

Merck, Darmstadt

Gibco BRL, Eggenstein

Chemicon, Temecula, USA

Sigma, Deisenhofen

Merck, Darmstadt

Merck, Darmstadt

Gibco BRL, Eggenstein

Sigma, Deisenhofen

Merck, Darmstadt

PAN, Aidenbach 
Penicillin/Streptomycin

Peptone

Restriction enzymes

Retinoic acid

Reverse transcriptase

RNase away

S.O.C medium

SDS

SeeBlue Plus2 Pre-Stained Standart

Tris / $\mathrm{HCl}$

Tris base

TritonX-100

TrypLE Express (Trypsin)

Trypsin / EDTA

Yeast extract

X-Gal

\subsubsection{Solutions and buffers}

\subsubsection{Agarose gel electrophoresis}

$5 \times$ TBE buffer

$445 \mathrm{mM}$ Tris base

$445 \mathrm{mM}$ Boric acid

10 mM EDTA (pH 8)

DNA loading buffer

15\% Ficoll 400

$1 \mathrm{mM}$ EDTA

$0.01 \%$ Orange G

10\% Glycerin 


\subsubsection{Frequently used buffers and solutions}

TE buffer $10 \mathrm{mM}$

dNTP-solution (25 mM)

IPTG

P1-buffer

P2-buffer

P3-buffer

X-Gal-solution

DEPC-water

Gelatin $0.1 \%$
Tris/HCl (pH 8.0)

$1 \mathrm{mM}$ EDTA

$100 \mathrm{mM}$ dATP

$100 \mathrm{mM}$ dCTP

$100 \mathrm{mM}$ dGTP

$100 \mathrm{mM}$ dTTP

$100 \mathrm{mM}$ in $\mathrm{H}_{2} \mathrm{O}$

$50 \mathrm{mM}$ Tris $/ \mathrm{HCl}(\mathrm{pH} 8,0)$

$10 \mathrm{mM}$ EDTA

$100 \mu \mathrm{g} / \mathrm{ml}$ RNaseA

$200 \mathrm{mM} \mathrm{NaOH}$

$1 \%$ SDS

3,2 M Potassium acetate $(\mathrm{pH} 5,0)$

2\% X-Gal in Dimethylformamide

$0.1 \%(\mathrm{v} / \mathrm{v})$

Diethylpyrocarbonate

$0.1 \%$ diluted in Ampuwa

water and autoclaved 


\subsubsection{Media and cells}

\subsubsection{Eukaryotic cell culture media and cells}

Cell lines:

\begin{tabular}{|c|c|}
\hline Cell line & Reference/Source \\
\hline \multirow{2}{*}{ ESC R1 } & Wurst and Joyner, 1993 \\
\hline & Cell Bank of the Institute of Human Genetics in Göttingen \\
\hline \multirow{2}{*}{ maGSC $129 \mathrm{SV}$} & Guan et al., 2006 \\
\hline & Cell Bank of the Institute of Human Genetics in Göttingen \\
\hline EGC & Stewart et al., 1994 \\
\hline \multirow{2}{*}{$3 \mathrm{~T} 3$ (NIH3T3) } & Todaro and Green, 1965 \\
\hline & Cell Bank of the Institute of Human Genetics in Göttingen \\
\hline
\end{tabular}

ESC medium

DMEM

$0,1 \mathrm{mM}$ non-essential amino-acids

$1 \mathrm{mM}$ Sodium pyruvate

$1 \mu \mathrm{M} \beta$-Mercaptoethanol

2 mM L-Glutamine

$1 \mathrm{mM}$ Penicillin / Streptomycin

$20 \%$ FCS

(1000 U / ml LIF)

FB medium

DMEM

2 mM L-Glutamine

$1 \mathrm{mM}$ Penicillin / Streptomycin

$10 \%$ FCS 
FB freezing medium $30 \%$ FB-Medium

20\% DMSO

$50 \%$ FKS

ESC freezing medium

30\% ESC-Medium

20\% DMSO

$50 \%$ FKS

\title{
2.1.6.2 Bacteria culture media and cells
}

Bacteria:

\begin{tabular}{|l|l|}
\hline Escherichia coli DH5 $\alpha$ & Invitrogen, Karlsruhe \\
\hline
\end{tabular}

LB-medium (pH 7,5)

\author{
Peptone $5 \mathrm{~g}$ \\ Yeast extract $2,5 \mathrm{~g}$ \\ $\mathrm{NaCl} 5 \mathrm{~g}$ \\ $500 \mathrm{ml} \mathrm{H}_{2} \mathrm{O}$
}

S.O.C.-medium

Invitrogen, Karlsruhe

For selection

Ampicillin (0,1 mg / ml)

For Oja-Plates

$100 \mu \mathrm{M}$ IPTG

0,4\% X-Gal 


\subsubsection{Vectors}

pGEM-T easy

Promega, Madison, USA

pMIR-REPORT ${ }^{\mathrm{TM}}$ miRNA

Ambion (Applied Biosystems),

Expression Reporter Vector System

Darmstadt

\subsubsection{Enzymes (with supplied buffers)}

Restriction enzymes

Invitrogen, Karlsruhe

\subsubsection{Oligonucleotides}

Primer name
mOct4-F
mOct4-R
mBrachyury-F
mBrachyury-R
mEomesodermin-F
mEomesodermin- $\mathrm{R}$
mFgf8-F
mFgf8-R
mNestin-F
mNestin-R
mDppa3-F
mDppa3-R

Sequence (5' to 3')

AAAGCAACTCAGAGGGAACCTCCTC

GCTTGGCAAACTGTTCTAGCTCCTTC

CAATGGAGGGGGACAGATCAT

AAGGGCTGTAATCTCCTCATTCTG

CATGCAGGGCAATAAGATGTACG

GTATTTGTGCAGAGACTGCAACAC

CTGAGCTGCCTGCTGTTGCACTTG

GCTCTGCTCCCTCACATGCTGTGT

CTGCAGGCCACTGAAAAGTT

ATTAGGCAAGGGGGAAGAGA

GCAGTCTACGGAACCGCATTG

GGCAGAAAGTGCAGAGACATC 
mSdha-F

GCTTGCGAGCTGCATTTGG

mSdha-R

CATCTCCAGTTGTCCTCTTCCA

\subsubsection{Kits}

Attractene Transfection Reagent

$\mathrm{Cy}^{\mathrm{TM}} 3$ dye-labeled Pre-miR ${ }^{\mathrm{TM}}$

Negative Control

DYEnamic ET-Terminator Mix

Galacto-Light System Plus

HiPerFect Transfection Reagent

Luciferase Assay System with

Reporter Lysis Buffer

miRCURYTM LNA microRNA

Array microarray kit and power

labeling kit

miRNeasy mini Kit

miScript Primer Assays

miScript Reverse Transcription Kit
Qiagen, Hilden

Ambion (Applied Biosystems),

Darmstadt

Amersham, Freiburg

Applied Biosystems, Darmstadt

Qiagen, Hilden

Promega, Madison, USA
Qiagen, Hilden

Qiagen, Hilden

Qiagen, Hilden 
miScript SYBR Green PCR Kit

Montage PCR

pGEM ${ }^{\circledR T}$-easy Vector System I

Pre-miR ${ }^{\mathrm{TM}}$ miRNA Precursor

Molecules

(miR-302a, miR-302b, miR-302d)

QIAGEN Plasmid Mini Kit

Qiaquick Gel Extraction Kit

QuantiTect SYBR-Green PCR

Master Mix
Qiagen, Hilden

Millipore, Karlsruhe

Promega, Madison, USA

Ambion (Applied Biosystems),

Darmstadt

Qiagen, Hilden

Qiagen, Hilden

Qiagen, Hilden 


\subsection{Animal cell culture methods}

\subsubsection{Animal cell culture protocols}

This section describes the working procedures in the animal cell culture laboratory. A protocol on aseptic technique was followed in order to handle the threat of microbial contamination and to avoid cross contamination between different cell lines. Cells were cultured in culture flasks or plates at $37^{\circ} \mathrm{C}$ in a humidified atmosphere of $5 \% \mathrm{CO}_{2}$.

\subsubsection{Resuscitation of frozen cells}

All cell lines that were used were obtained from the institute's culture collection. In order to avoid cell toxicity of DMSO above $4^{\circ} \mathrm{C}$, the frozen cells were quickly thawed at $37^{\circ} \mathrm{C}$ water bath and dropwise pipetted into $9 \mathrm{ml}$ of prewarmed growth medium. The cells were then spinned down in $300 \mathrm{x} \mathrm{g}$ for $5 \mathrm{~min}$ and the supernatant was discarded by aspiration. Cells were resuspended in a suitable amount of prewarmed growth medium and plated in the appropriate flask or plate.

\subsubsection{Subculture of adherent cell lines}

Before confluence was reached, cells were subcultured. Cells were washed once with sterile PBS using a volume equivalent to the volume of the culture medium. In order to be brought into suspension, cells were then incubated in trypsin (TrypLE ${ }^{\mathrm{TM}}$ Express), in an amount of approximately $1 \mathrm{ml}$ per $25 \mathrm{~cm}^{2}$, at $37^{\circ} \mathrm{C}$ 
until they had detached from the dish (5-10 min). Cells were examined under an inverted microscope to ensure that all cells were detached. Trypsin was inactivated by addition of fresh growth medium in which the cells were subsequently resuspended. 100-200 $\mu$ were removed and cell counting was performed, when necessary, using an improved Neubauer chamber (see section 2.2.4). Then the cells were plated out or harvested for cryopreservation.

For subculture resuspended cells were spun down $(300 \mathrm{x}$ g for $5 \mathrm{~min}$ at room temperature). The supernatant was aspirated and the cells were resuspended in the suitable amount of prewarmed medium and were then transferred to new labeled flasks containing pre-warmed medium.

For cryopreservation resuspended cells were spun down ( $300 \mathrm{x} g$ for $5 \mathrm{~min})$. The supernatant was aspirated and the cells were resuspended in the suitable amount of prewarmed medium. To the resuspended cells an equal volume of freezing medium was added. $1 \mathrm{ml}$ aliquots of cells were transferred into labeled cryoprotective ampules. Cells were kept for 7 days at $-80^{\circ} \mathrm{C}$, and then stored in liquid nitrogen.

\subsubsection{Cell quantification}

After resuspension of adherent cells with trypsin (see section 2.2.3), 100-200 uL of cell suspension were removed and an equal volume of trypan blue was added. Both sides of the chamber were filled (10 $\mu \mathrm{l}$ each) with cell suspension and viewed under a light microscope using x 20 magnification. The number of viable cells (seen as bright cells) and non-viable cells (stained blue) - was counted. The concentration of viable and non-viable cells and the percentage of viable cells were calculated using the equations provided by the haemocytometer manufacturer. 


\subsubsection{Mouse Embryonic Fibroblasts (MEFs) and feeder layers}

For isolation of MEFs pregnant mice at day 13.5-15.5 p.c. were used. Using sterile forceps and scissors the abdominal cavity was opened and both uterine horns were dissected and placed in a Petri dish. After excess fat and mesentery were trimmed away, horns were opened under microscope and the conceptuses were released and transferred into a fresh dish containing PBS. Using forceps the embryonic membranes were opened, head was pinched off and internal organs were removed. The remaining was transferred into a conical glass tube containing small glass balls and $0.125 \%$ trypsin/0.01\% EDTA and incubated with magnetic agitation at $37{ }^{\circ} \mathrm{C}$ for $20-40 \mathrm{~min}$. Then FB medium was added to stop trypsinization and cells were centrifuged for $10 \mathrm{~min}$ with $1000 \mathrm{rpm}$. Pellet was resuspended in FB medium and cells were subsequently plated. When these flasks of primary MEFs were confluent, cells were passaged one more time before freezing and stored under liquid nitrogen in FB freezing medium.

When needed, cells were thawed and expanded for four more passages in gelatine coated flasks. For preparation of feeder layer, inactivation was performed with $100 \mu \mathrm{l}$ of $1 \mathrm{mg} / \mathrm{ml}$ mitomycin C stock solution in $10 \mathrm{ml} \mathrm{FB}$ medium for 3 hrs. Cells were washed with sterile PBS and passaged into new gelatine coated flasks for direct use as feeder layer after one day. 


\subsubsection{Culture of ESCs, maGSCs and EGCs}

After thawing of the frozen ESCs/maGSCs/EGCs in ESC medium on feeder layer-coated flasks, cells were expanded for 1-2 passages before beginning of the experiments. Cells were passaged after they had reached $80 \%$ of confluency. Flasks were seeded as follows: T25 flask, 1-1.5 x 106 cells, 6 well plates, 4-5 x $10^{4}$ cells. For maintenance in pluripotent state, cells were cultured on feeder layer in ESC medium in the presence of $1000 \mathrm{U} / \mathrm{ml}$ recombinant mouse Leukemia Inhibitory Factor (LIF). Undifferentiated phenotype of the colonies was monitored daily under the microscope.

\subsubsection{Culture of ESCs, maGSCs and EGCs: differentiation conditions}

For the differentiation studies, the following conditions were used:

A. Cells were cultured in $0.1 \%$ gelatin coated culture flasks with ESC medium, without LIF (gelatine condition);

B. Cells were cultured in $0.1 \%$ gelatin coated culture flasks with ESC medium, without LIF but with RA $10^{-6} \mathrm{M}$ (gelatine and RA condition).

\subsubsection{Culture of NIH/3T3 cells}

NIH/3T3 cells were cultured in FB medium in appropriate culture flasks. 


\section{3 miRNA array expression profiling}

For the miRNA array expression profiling two kits were used:

I. the miRCURYTM LNA microRNA Array power labeling kit (Exiqon), for labeling of RNA molecules, and

II. the miRCURYTM LNA microRNA Array microarray kit (Exiqon).

Hybridizations and scanning of the slides were carried out in the special equipped ozone free laboratory of the Microarray Facility of University of Goettingen. Experiment design, image acquisition and quantification were performed in cooperation with Dr. Salinas and Mr. Opitz of this lab.

\subsubsection{Labeling of RNA molecules}

The miRCURYTM LNA microRNA Array Power labeling kit was applied for labeling RNA molecules with a single fluorophore per molecule following a 2step protocol provided by the manufacturer. The first step includes a Calf Intestinal Alkaline Phosphatase for removal of 5'-phosphates from terminal of the miRNAs (CIP treatment). In the second step, a fluorescent label is attached enzymatically to the 3'-end of the miRNAs in the total RNA sample. This is followed by an enzyme inactivation step after which the sample is ready for hybridization.

In all experiments a dual color hybridization with $\mathrm{Hy} 3^{\mathrm{TM}}$ and $\mathrm{Hy} 5^{\mathrm{TM}}$ labeled RNA was performed, in which each CIP treated RNA sample was labeled with either Hy $3^{\mathrm{TM}}$ or $\mathrm{Hy} 5^{\mathrm{TM}}$ (dyes are equivalent to $\mathrm{Cy}-3$ and $\mathrm{Cy}-5$ dyes). $1 \mu \mathrm{g}$ of total RNA containing small RNAs (and miRNAs) was used per labeling reaction per slide hybridization according to manufacturer's recommendations. 
To assure optimal labeling and hybridization, spike-in control probes (Spike-in miRNA kit) supplied in the miRCURYTM LNA microRNA Array microarray kit were used. The miRCURYTM LNA microRNA Array Spike-in kit contains 10 different synthetic unlabeled miRNAs in different concentrations. The set was spiked into each RNA sample prior to labeling so that the synthetic Spike-in miRNAs would hybridize to corresponding capture probes on the miRCURYTM LNA microRNA Array. Spike-in miRNAs are provided at concentrations compatible with endogenous miRNA expression levels aimed at spanning the whole intensity range of miRNAs in most tissue samples. The corresponding capture probes are printed once in every subgrid, thus 32 times each.

The spike-in miRNAs were added in equal amounts to labeling reactions before the dual-color array hybridizations, so that the signals from the spike-in capture probes could be used as a control of the labeling reaction and hybridization and as a help in deciding scanner settings between channels. In addition they were used as a control of the data normalization procedure, to estimate the variance of replicated measurements within arrays and to assess technical variability between different parts of the array.

\subsubsection{Description of miRCURY ${ }^{\mathrm{TM}}$ LNA microRNA Array slides}

The miRCURYTM LNA microRNA Array slides contain capture probes complementary to all mature miRNAs in human, rat and mouse as annotated in miRBase release 9.2. The capture probes are Locked Nucleic Acid (LNA ${ }^{\mathrm{TM}}$ ) enhanced oligonucleotides, Tm-normalized to hybridise optimally under the protocol conditions. In addition, a number of capture probes are available for detection of miRNAs not included in miRBase (miRPlus ${ }^{\mathrm{TM}}$ probes). 
In addition, thirty control capture probes were included in the probe set as follows:

1. Ten spike-in control probes to assure optimal labeling and hybridization (mentioned above).

2. Eight negative capture probes.

3. Twelve capture probes are included that hybridize to small nuclear RNAs.

Design layout of the experiments, with arrows representing hybridizations, is also shown in the results section.

The miRCURYTM LNA microRNA Array layout was as follows:

The array was located on a standard size slide as illustrated in the drawing below (25.4 $\mathrm{mm}$ by $76.2 \mathrm{~mm}$ or 1 in by $3 \mathrm{in}$ ). The array carries the following specifications:

- Outer dimensions: $17 \mathrm{~mm}$ wide by $37 \mathrm{~mm}$ long

- Coordinates of first spot on slide $=4 \mathrm{~mm}, 15 \mathrm{~mm}$

- 8 sub-arrays in 4 replicates

- Spot size: $90 \mu \mathrm{m}$

- Distance between spots: $225 \mu \mathrm{m}$

\subsubsection{Hybridization and washing of the arrays}

Hybridization was carried out manually using an Agilent hybridization SureHyb chamber kit and gasket slide kit in hybridization buffer supplied with the kit at $56^{\circ} \mathrm{C}$ for $16 \mathrm{hrs}$ in a hybridization oven with rotation. Washing was carried out 
the next day according to manufacturer's recommendations and then slides were dried and scanned. For image acquisition, quantification, normalization of the results and statistical analysis the software packages provided by the Facility were used. This part of the experiment was carried out in cooperation with the bioinformatician of the Facility, Mr. L. Opitz.

\subsection{Transfection of animal cells with miRNAs}

\subsection{1 miRNA molecules used in cell transfection}

Cultured cells were transfected with Pre-miR ${ }^{\mathrm{TM}}$ miRNA Precursor Molecules. These are small, chemically modified double-stranded RNA molecules designed to mimic endogenous mature miRNAs. These ready-to-use miRNA mimics have been carefully designed and modified to ensure that the correct strand, representing the desired mature miRNA, is taken up into the RNA-Induced Silencing Complex (RISC) that is responsible for miRNA activity. In this study Pre-miR ${ }^{\mathrm{TM}}$ miRNA Precursor Molecules for miR-302a, miR-302b, miR-302c and miR-302d were applied on cultured cells that were then designated as positive probes (pos).

In addition, Pre-miR ${ }^{\mathrm{TM}}$ Negative Control was used. This is a random sequence Pre-miR molecule that has been extensively tested in cell lines and tissues and validated to not produce identifiable effects on known miRNA function. PremiR $^{\mathrm{TM}}$ Negative Control was used for transfection of cells that were cultured under exactly the same conditions as positive cell probes and were designated as the respective negative controls (neg). 
The lyophilized Pre-miR ${ }^{\mathrm{TM}}$ miRNA Precursor Molecules were resuspended in $100 \mu \mathrm{l}$ of RNase-free water (stock solution with concentration of $50 \mu \mathrm{M}$ ). To $10 \mu 1$ of the stock solution another $240 \mu \mathrm{l}$ of RNase-free water were added and the resulting working solution (concentration of $2 \mu \mathrm{M}$ ) was used for transfection.

The used Pre-miR Negative Control is $\mathrm{Cy}^{\mathrm{TM}} 3$ dye-labeled at its $5^{\prime}$ end. This fluorescent label enables direct observation of the cellular uptake, distribution and localization of the control. Therefore this molecule was also used for monitoring transfection efficiency in preliminary transfection experiments (see 2.6.2).

\subsubsection{Optimization of transfection conditions}

Cell transfection was conducted with the use of HiPerFect Reagent (see in 2.6.3), which enables highly efficient miRNA transfection achieved in a range of 1-50 nM miRNA concentrations. Transfection of synthetic miRNAs can result in off-target effects. These can include mRNA degradation, inhibition of translation or induction of an interferon response. Therefore, the optimal miRNA concentration has to be determined. This concentration depends on miRNA potency, cell type and targeted genes.

For transfection optimization $\mathrm{Cy}^{\mathrm{TM}} 3$ Pre-miR Negative Control was applied in ESCs in different concentrations. One and two days after the transfection transfected cells were evaluated with the use of fluorescence microscope. By microscopically efficient transfection FACS was performed in order to determine the exact transfection rate. Moreover, ESCs were retransfected two days after the initial transfection and evaluated microscopically and by FACS analysis in order to establish the optimal frequency of retransfections. 


\subsubsection{Transfection procedure}

For cell transfection HiPerFect Reagent was used according to Fast-Forward Protocol for transfection of adherent cells adjusted to 6-well culture plates. In this protocol cell plating and transfection are performed on the same day.

On day 0 shortly before transfection $5 \times 10^{5}$ cells were plated in every well of a 6-well plate in $2,3 \mathrm{ml}$ of the appropriate culture medium and cells were incubated under normal growth conditions $\left(37^{\circ} \mathrm{C}\right.$ and $\left.5 \% \mathrm{CO}_{2}\right) .12 \mu \mathrm{l}$ of working solution for each of Pre-miRTM miRNA Precursor of miR-302 cluster were diluted to $34 \mu \mathrm{l}$ DMEM. To this $18 \mu \mathrm{l}$ of HiPerFect were added to a final volume of $100 \mu$ l. The content was mixed thoroughly by pipetting and was incubated at room temperature for 5-10 min to allow the formation of transfection complexes. Culture medium was aspirated from the cells and $0,5 \mathrm{ml}$ of fresh culture medium was added. Then the complexes were added drop-wise onto cells. The plate was gently swirled to ensure uniform distribution of transfection complexes and cells were further incubated under normal growth conditions for $24 \mathrm{hrs}$. Cells were passaged (1:3) and retransfected as described every two days.

\subsection{Co-transfection of cells with miRNAs and plasmid DNA}

\subsection{1 miRNA/DNA co-transfection: overview}

For co-transfection of cells with miRNAs and plasmid DNA Attractene Transfection Reagent was used. This is a nonliposomal lipid which enables highly efficient DNA transfection, ensuring low cytotoxicity, which is critical for successful transfection experiments. Attractene Reagent is a one-tube reagent 
and reduces protocol steps. It enables also transfection in the presence of serum without lowering transfection efficiency.

In this study the Traditional Protocol, as given by supplier, was performed, adjusted to co-transfection conditions of DNA plasmids and miRNAs. $3 \times 10^{4}$ NIH3T3 cells were plated in every well of a 24 -well culture plate $24 \mathrm{hrs}$ prior to transfection. Cells were seeded in $0,5 \mathrm{ml}$ of appropriate culture medium and incubated under normal growth conditions $\left(37^{\circ} \mathrm{C}\right.$ and $\left.5 \% \mathrm{CO}_{2}\right)$. On the day of transfection cells had reached a confluency of $70-80 \%$, as evaluated by examination with an inverted microscope. This confluency lies in the optimal range that should be achieved at the time of complex addition.

\subsubsection{Transfection procedure}

0,4 $\mu \mathrm{g}$ of miRNA Luciferase Vector (containing the 3' UTR of the gene that was at the time investigated), $0,4 \mu \mathrm{g}$ of $\beta$-galactosidase control plasmid (for description of vectors see 2.6.1) and totally $0,5 \mu \mathrm{g}$ of Pre-miR ${ }^{\mathrm{TM}}$ miRNA Precursor Molecules for miR-302a, miR-302b, miR-302c and miR-302d (for description see 2.4.1) were diluted in culture medium to a total volume of $60 \mu$. 1,5 $\mu 1$ of Attractene Transfection Reagent was added to the DNA/RNA solution. The solution was mixed thoroughly by pipetting and incubated for 10-15 $\mathrm{min}$ at room temperature to allow complex formation. Culture medium was aspirated from the cells and $0,5 \mathrm{ml}$ of fresh culture medium was added. Transfection complexes were drop-wise added onto the cells. The plate was gently swirled to ensure uniform distribution of transfection complexes and cells were further incubated under normal growth conditions for $24 \mathrm{hrs}$. 


\section{6 pMIR-REPORT ${ }^{\mathrm{TM}}$ miRNA Expression Reporter Vector System, Luciferase assay system and ß-galactosidase assay system}

\subsection{1 pMIR-REPORT ${ }^{\mathrm{TM}}$ miRNA Expression Reporter Vector System}

pMIR-REPORT ${ }^{\mathrm{TM}}$ miRNA Expression Reporter Vector System consists of an experimental firefly Luciferase reporter vector (miRNA Luciferase Vector) and an associated $\beta$-galactosidase reporter control plasmid. By inserting predicted miRNA target sequences in the multiple cloning site, which is located downstream of the Luciferase ORF, miRNA Luciferase Vector can be used to examine miRNA function, to evaluate possible miRNA target sites and to investigate the influence of 3' UTR sequences on gene expression. The vector contains a firefly Luciferase reporter gene under the control of a CMV promoter/termination system (Scheme 1). The 3' UTR of the Luciferase gene contains a multiple cloning site for insertion of predicted miRNA binding targets. By cloning a predicted miRNA target sequence into miRNA Luciferase Vector, the Luciferase reporter is subjected to regulation that mimics the miRNA target. 


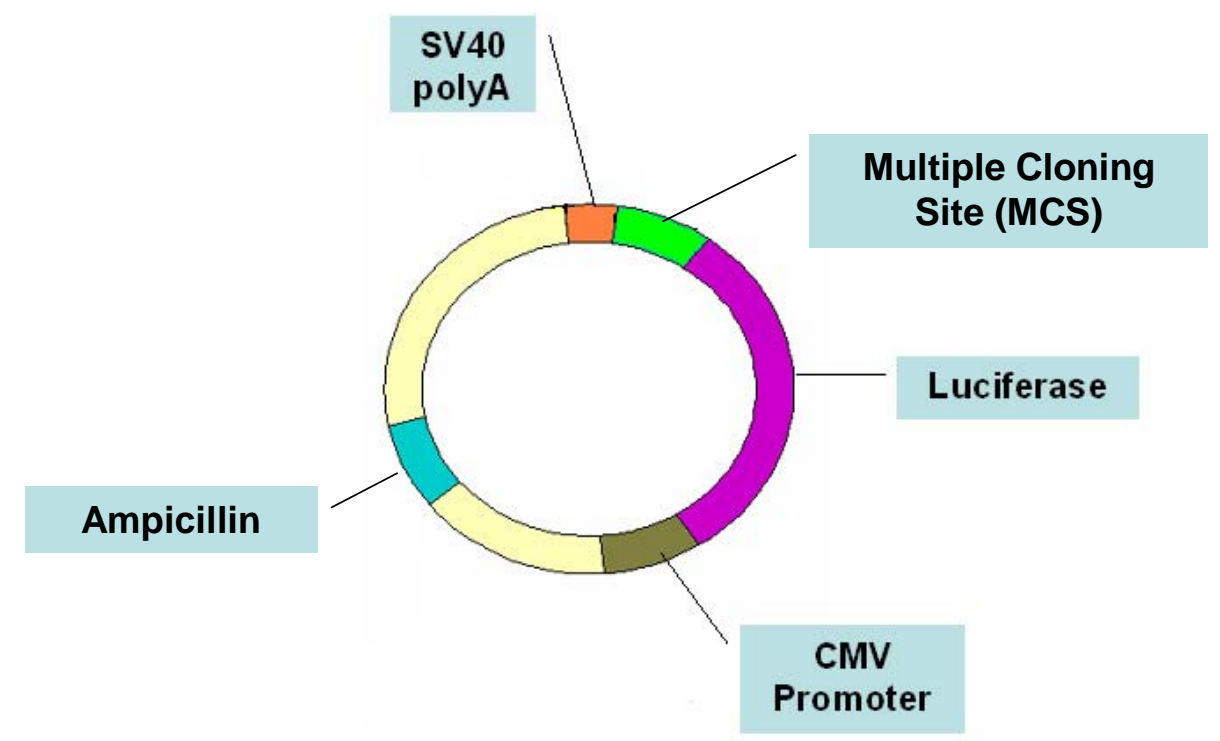

Scheme 1. miRNA Luciferase Vector. The miRNA Luciferase Vector expresses firefly Luciferase (pink) under the control of CMV promoter (brown). A multiple cloning site (MCS) (green) and a SV40 polyadenylation signal (orange) are located downstream of the Luciferase translation sequence.

The $\beta$-galactosidase control plasmid of the used system is a $\beta$-galactosidase reporter that is designed for transfection normalization. ß-galactosidase expression from this control plasmid was used to normalize variability due to differences in cell viability and transfection efficiency.

\subsubsection{Luciferase assay system}

Luciferase assay system detection of firefly Luciferase activity is used to study gene expression. This system consists of the Luciferase assay reagent and the $5 \mathrm{x}$ Reporter Lysis Buffer (RLB). The Luciferase assay reagent is prepared by addition of Luciferase assay buffer to the lyophilized Luciferase assay substrate according to manufacturer's recommendations. 
Luciferase Assay Reagent contains luciferin, the substrate of firefly luciferase. This enzyme, produced in miRNA Luciferase Vector-transfected cells, is a monomeric $61 \mathrm{kDa}$ protein that catalyzes the oxidation of luciferin using ATP$\mathrm{Mg}^{2+}$ as a cosubstrate. Light is produced by converting the chemical energy of luciferin oxidation through an electron transition, forming the product molecule oxyluciferin according to the following reaction (Fig. 2):

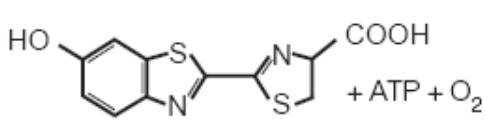

Beetle Luciferin

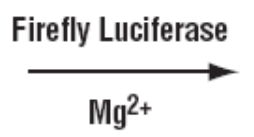

$\mathrm{Mg}^{2+}$

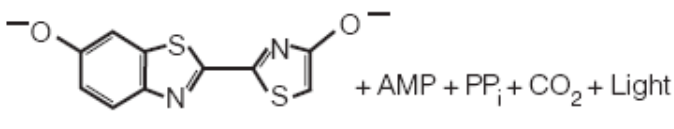

Oxyluciferin

Figure 2. Bioluminescent reaction catalyzed by firefly luciferase.

\subsection{3 ß-galactosidase assay}

The Galacto-Light Plus ${ }^{\mathrm{TM}}$ B-Galactosidase Assay System was used for detection of ß-galactosidase reporter enzyme in cell lysates. The Galacto-Light ${ }^{\mathrm{TM}}$ Plus $^{\mathrm{TM}}$ system incorporates chemiluminescent substrates, reaction buffer and lightemission accelerator containing luminescence enhancers in a ready-to-use format. The reaction buffer is supplied at a $\mathrm{pH}$ that aids in discrimination of endogenous $\beta$-galactosidase, while having little effect on the bacterial form of the enzyme. This enables sensitive detection even in cell lines with relatively high levels of endogenous $\beta$-galactosidase activity.

The substrate contained in the reaction buffer is cleaved by $\beta$-galactosidase enzyme during incubation for $1 \mathrm{~h}$. By addition of the light-emission accelerator the enzyme activity is terminated and light emission from the cleaved substrate is triggered. 


\subsubsection{Preparation of cell lysates}

Reporter Lysis Buffer (RLB) is a mild lysis agent and requires a single freezethaw cycle to achieve complete cell lysis. For preparing cell lysates 4 volumes of dd $\mathrm{H}_{2} \mathrm{O}$ are added to 1 volume of 5x RLB. Before use 1x lysis buffer is equilibrated to room temperature. After aspirating culture medium from the cells and washing them with PBS, $100 \mu 1$ of $1 \mathrm{x}$ lysis buffer was added to every well of the 24-well culture plate, so that the cells were covered. To ensure complete lysis a single freeze-thaw cycle in $-80{ }^{\circ} \mathrm{C}$ was performed. After thawing, cells were scraped from culture plate in order to detach completely and they were transferred in a microcentrifuge tube. The tube was vortexed for 10-15 sec and then centrifuged for $2 \mathrm{~min}$ at $4{ }^{\circ} \mathrm{C}$. The supernatant was finally transferred in a new microcentrifuge tube and stored at $-70{ }^{\circ} \mathrm{C}$ or used for measurement of luciferase and $\beta$-galactosidase activity in a plate-reading luminometer.

\subsubsection{Measurement in plate-reading luminometer with injector}

Cell lysate was placed in a microtiter plate. Wells for luciferase activity measurement contained $20 \mu \mathrm{l}$ of cell lysate. The injector added $100 \mu \mathrm{l}$ of Luciferase assay reagent per well, then each well was read immediately. The luminometer was programmed for delay time of $2 \mathrm{sec}$ and a measurement period of luciferase activity of $10 \mathrm{sec}$.

In case of $\beta$-galactosidase assay the reaction buffer was prepared according to supplier's recommendations and both reaction buffer and light-emission accelerator were equilibrated to room temperature. $10 \mu \mathrm{l}$ of cell lysate were placed in the microtiter plate and $70 \mu \mathrm{l}$ of reaction buffer were injected in each well. The luminometer was programmed for a delay of $14 \mathrm{sec}$ followed by 
measurement of background B-galactosidase activity for $1 \mathrm{sec}$. After incubation of $1 \mathrm{~h} 100 \mu \mathrm{l}$ of light-emission accelerator were injected in every well. After delay time of $5 \mathrm{sec}$ the signal was read for $10 \mathrm{sec} / \mathrm{well}$.

\subsubsection{Data analysis}

Luciferase activity levels were calculated by determining the ratio between the level of luciferase activity and level of ß-galactosidase activity in every sample. Average values were used because the measurements were performed in replicates. This ratio was then compared between different samples. For this reason the normalized value of one sample was set to 1 (calibrator sample) and relative luciferase activity in samples of interest was compared by dividing the normalized sample values by the value of the calibrator.

\subsection{RNA isolation}

\subsubsection{Isolation of total RNA including small RNAs}

Most commercial RNA purification kits do not recover such small RNA molecules like miRNAs. For this reason the miRNeasy Mini Kit was used for purification of total RNA, including miRNAs and other small RNA molecules.

The method used by this kit combines phenol/guanidine-based lysis of samples and silica-membrane-based purification of total RNA. The lysis reagent which is included in the kit is a monophasic solution of phenol and guanidine thiocyanate, designed to facilitate lysis of tissues, to inhibit RNases, and also to 
remove most of the cellular DNA and proteins from the lysate by organic extraction.

Determination of the correct amount of starting material (animal cells) was based on manufacturer's recommendations, so that the correct volume of lysis reagent required for efficient lysis is used and the RNA binding capacity of the column is not exceeded. For this reason not more than $3-4 \times 10^{6}$ cells were used. Cells were trypsinized and counted (see section 2.2.4). After inactivation of the trypsin with serum-conaining medium, cells were transferred into an RNase-free polypropylene centrifuge tube and spun down at $300 \mathrm{x} \mathrm{g}$ for $5 \mathrm{~min}$ at room temperature. After washing with PBS, cells were collected as pellet and homogenized by vortexing in $700 \mu$ lysis reagent (provided with the kit).

After addition of $140 \mu \mathrm{l}$ chloroform, the homogenate was separated into aqueous and organic phases by centrifugation at $12000 \mathrm{x}$ for $15 \mathrm{~min}$ at $4^{\circ} \mathrm{C}$. RNA partitions to the upper, aqueous phase, DNA partitions to the interphase and proteins to the lower, organic phase or the interphase. The upper, aqueous phase was extracted and ethanol (1,5 volumes) was added to provide appropriate binding conditions for all RNA molecules from 18 nucleotides (nt) upwards.

The sample was then applied to the RNeasy Mini spin column, where the total RNA binds to the membrane and phenol and other contaminants are efficiently washed away with the provided RWT and RPE buffers according to manufacturer's protocols. RNA was then eluted in $30 \mu \mathrm{l}$ RNase-free water. To obtain a higher total RNA concentration, a second elution step was performed by using the first eluate. The isolated RNA was stored at $-80^{\circ} \mathrm{C}$. 


\subsubsection{Determination of nucleic acid concentration}

The concentration of nucleic acids was determined spectrophotometrically by measuring absorption of the samples at $260 \mathrm{~nm}$. The quality of nucleic acids i.e. contamination with salt and protein was checked by measurements at 260, 280, and $320 \mathrm{~nm}$. The concentration was calculated according to the formula:

$\mathrm{C}=(\mathrm{E} 260-\mathrm{E} 320) \mathrm{fc}$

$\mathrm{C}=$ concentration of sample $(\mu \mathrm{g} / \mu \mathrm{l})$

E $260=$ ratio of extinction at $260 \mathrm{~nm}$

E $320=$ ratio of extinction at $320 \mathrm{~nm}$

$\mathrm{f}=$ dilution factor

$\mathrm{c}=$ concentration (standard) / absorption (standard)

for RNA : $\mathrm{c}=0.04 \mu \mathrm{g} / \mu \mathrm{l}$

\section{8 miRNA and mRNA expression}

miScript System was used for detection and quantification of miRNAs. The miScript System consists of:

I. miScript Reverse Transcription Kit,

II. miScript Primer Assays (only for miRNAs) and

III. miScript SYBR ${ }^{\circledR}$ Green PCR Kit (only for miRNAs).

Reverse Transcription PCR (RT-PCR) is a technique, which generates cDNA fragments from RNA templates. Generated cDNA can be thereafter amplified by PCR. In the first step of RT-PCR, called the "first strand reaction", cDNA is made from an mRNA template using dNTPs and an RNA-dependent DNA polymerase (reverse transcriptase) through the process of reverse transcription. RT-PCR exploits a characteristic of mature mRNAs known as the $3^{\prime}$ 
polyadenylated region, commonly called the poly(A) tail, as a common binding site for poly(T) DNA primers.

\subsubsection{Conversion of miRNAs and other small non-coding RNAs into cDNA (reverse-transcription step)}

Unlike mRNAs, miRNAs are not polyadenylated. During the reverse transcription step using miScript Reverse Transcription Kit miRNAs are polyadenylated by poly(A) polymerase. Reverse transcriptase converts RNA (including miRNA, other small noncoding RNA, and mRNA) to cDNA using oligo-dT and random primers. Polyadenylation and reverse transcription are performed in parallel in the same tube. The oligo-dT primers used in the reverse transcription step have a universal tag sequence on the $5^{\prime}$ end, which allows amplification in the next step (Real-Time PCR step)

$1 \mu \mathrm{g}$ of total RNA containing miRNAs was mixed with $4 \mu 1$ of miScript Buffer (room temperature) and $1 \mu \mathrm{l}$ miScript Reverse Transcriptase Mix. The reaction mix was made with the addition of RNase-free water up to a total volume of 20 $\mu 1$. Samples were further incubated at $37^{\circ} \mathrm{C}$ for $60 \mathrm{~min}$ for the first strand cDNA synthesis. Next, the reaction was inactivated by heating at $95^{\circ} \mathrm{C}$ for $15 \mathrm{~min}$ and stored at $-20^{\circ} \mathrm{C}$. 


\subsection{2 cDNA amplification by PCR}

Polymerase chain reaction (PCR) is a method that allows logarithmical amplification of short DNA sequences within a longer double stranded DNA molecule. After several (often 33-40) rounds of amplification, the PCR product is analyzed on an agarose gel and is abundant enough to be detected with an ethidium bromide stain.

In this work, semi-quantitative PCR was used preliminary to establish the optimal conditions and identify the right positive controls used later in qPCR. This method can be used as a qualitative tool for detecting the presence or absence of a particular DNA.

The PCR reaction contains in general the following substances in a $200 \mu \mathrm{l}$ reaction tube: 1-10 ng template DNA, $1 \mu$ forward primer $(10 \mathrm{pmol} / \mu \mathrm{l}), 1 \mu \mathrm{l}$ reverse primer $(10 \mathrm{pmol} / \mu \mathrm{l}), 0,3 \mu \mathrm{l}$ magnesium chloride $\left(\mathrm{MgCl}_{2}\right)$ and $5 \mu \mathrm{SYBR}$ Green PCR Master Mix. The reaction mix was made with the addition of dd $\mathrm{H}_{2} \mathrm{O}$ up to a total volume of $10 \mu \mathrm{l}$ and placed in the thermocycler.

A standard PCR program is as follows: $50^{\circ} \mathrm{C}$ for $2 \mathrm{~min}$, initial denaturation $95^{\circ} \mathrm{C}$ for $15 \mathrm{~min}$ followed by $30-35$ cycles of: $95^{\circ} \mathrm{C}$ for $15 \mathrm{sec}$ (denaturation), $55-65^{\circ} \mathrm{C}$ for $30 \mathrm{sec}$ (annealing, temperature depends on primer's $\mathrm{Tm}$ value), $72^{\circ} \mathrm{C}$ for 30 sec (extension, time depends on the PCR product, as 1 min for $1 \mathrm{~kb}$ DNA). Then a final extension step followed at $72^{\circ} \mathrm{C}$ for $10 \mathrm{~min}$ and then a step at $4{ }^{\circ} \mathrm{C}$ for infinite time till the PCR tubes were taken from the PCR machine. The simplest formula for calculating the $\mathrm{Tm}$ (melting temperature) of the primers is: $\mathrm{Tm}=4^{\circ} \mathrm{C}$ $\mathrm{x}$ (number of G's and C's in the primer) $+2^{\circ} \mathrm{C} x$ (number of A's and T's in the primer). 


\subsubsection{Gel electrophoresis}

Gel electrophoresis is the technique by which a mixture of charged macromolecules (e.g. nucleic acids) is separated in an electrical field according to their mobility which is directly proportional to macromolecule's charge to mass ratio.

Agarose gels are used to electrophorese nucleic acid molecules from as small as 50 bases to more than 50 kilobases, depending on the concentration of the agarose and the precise nature of the applied electrical field (constant or pulse). $1-3,5 \mathrm{~g}$ of agarose were usually added in $100 \mathrm{ml} 0.5 \mathrm{x}$ TBE buffer, boiled in the microwave to dissolve the agarose, then cooled down to about $60^{\circ} \mathrm{C}$ before adding $3 \mu \mathrm{l}$ ethidium bromide $(10 \mathrm{mg} / \mathrm{ml})$. The $1-3,5 \%$ agarose gels were poured onto a horizontal gel chamber.

\subsubsection{Real-Time qRT-PCR for miRNAs (real-time qRT-PCR step)}

In contrast to regular RT-PCR and analysis by agarose gels, real-time qRT-PCR (quantitative RT-PCR) allows accurate quantification of starting amounts of cDNA targets. In real-time qRT-PCR using SYBR Green binding to amplified cDNA, we are simply measuring the fluorescence increase as the dye binds to the increasing amount of DNA in the reaction tube. SYBR Green binds to all double-stranded DNA molecules, emitting a fluorescent signal of a defined wavelength on binding. The excitation and emission maxima of SYBR Green are at $494 \mathrm{~nm}$ and $521 \mathrm{~nm}$, respectively, and are compatible for use with any Real-Time cycler. Detection takes place in the extension step of real-time qRTPCR. Signal intensity increases with increasing cycle number due to the accumulation of PCR product. 
The cDNA converted from total RNA containing miRNAs served as the template for real-time qRT-PCR analysis using the miScript Primer Assay in combination with the miScript SYBR Green PCR Kit. As it has been already mentioned, the oligo-dT primers contain a universal tag sequence on their end. In the real-time qRT-PCR step miRNAs are amplified using the miScript Universal Primer, which primes from this universal tag sequence, together with the miRNA-specific primer (miScript Primer Assay).

A single cDNA preparation is sufficient to interrogate multiple miRNAs by realtime qRT-PCR using different miRNA-specific primers. The cDNA can also be used for detection of mRNA using gene-specific primer pairs (see 2.5.5).

In this study miScript Primer Assays (available from Qiagen) for the following miRNAs were used:

I. For members of miR-290 cluster: 290_5p, 291_5p, 292_3p, 293, 294, 295.

II. For members of miR-17-92 cluster: 17, 19b, 20a, $20 \mathrm{~b}$.

III. For members of miR-302 cluster: 302a, 302b, 302d.

IV. For other miRNAs: 320, 711, 720.

RNU6B was used as endogenous reference for normalization. For standard curves samples of undifferentiated ESCs were used. Selection of the appropriate sample for the standard curve was based on preliminary experiments testing detection of expression of each miRNA by RT-PCR in the sample with the respective annealing temperature and for 35 cycles.

Real-time qRT-PCR was performed in the respective cycler. The PCR reaction contained: $1 \mu 1 \mathrm{cDNA}, 5 \mu \mathrm{l}$ x miScript SYBR Green PCR Master Mix, $1 \mu 1$ 10x miScript Universal Primer, $1 \mu$ 10x miScript Primer Assay and RNase-free 
water to a total volume of $10 \mu \mathrm{dd} \mathrm{H}_{2} \mathrm{O}$ and placed into the individual wells of a 384-well plate.

The PCR program was as follows: initial activation step $95^{\circ} \mathrm{C}$ for $15 \mathrm{~min}$ followed by 40 cycles at $94^{\circ} \mathrm{C}$ for $15 \mathrm{sec}$ (denaturation), $55^{\circ} \mathrm{C}$ for $30 \mathrm{sec}$ (annealing) and $70^{\circ} \mathrm{C}$ for $30 \mathrm{sec}$ (extension). Then a final dissociation curve (melting curve) was made and PCR plates were kept at $4^{\circ} \mathrm{C}$ till they were taken out from the PCR machine.

Nonspecific PCR products and primer-dimers can also contribute to the fluorescent signal. Therefore, high PCR specificity is required when using SYBR Green. A way to check that the correct fragments were amplified is to do a melting curve. After real-time qRT-PCR amplification, the machine was programmed to do a melting curve, in which the temperature is raised by a fraction of a degree and the change in fluorescence is measured. At the melting point, the two strands of DNA will separate and the fluorescence rapidly decreases. The software plots the rate of change of the relative fluorescence units (RFU) with time $(\mathrm{T})(-\mathrm{d}(\mathrm{RFU}) / \mathrm{dT})$ on the $\mathrm{Y}$-axis versus the temperature on the $\mathrm{X}$-axis, and this will peak at the melting temperature (Tm). If the peaks are not similar, this might suggest contamination, mispriming, primer-dimer artifacts etc. A primer-dimer artifact would give a peak with a lower melting temperature (because it is such a short DNA).

PCR specificity was checked i) by the melting curves mentioned above, ii) by gel electrophoresis and iii) by sequencing of the PCR products after gel

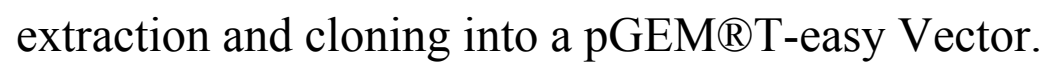




\subsubsection{Real-Time qRT-PCR for mRNAs (real-time qRT-PCR step)}

Real-time qRT-PCR for mRNAs was performed as for miRNAs with the following modifications: $5 \mu 1$ 2x QuantiTect SYBR Green PCR Master Mix , 1 $\mu 1$ 10x Forward Primer, $1 \mu 1$ 10x Reverse Primer, $0.3 \mu 1 \mathrm{MgCl}_{2}$ and RNase-free water to a total volume of $10 \mu \mathrm{l}$. The PCR program was as follows: $50^{\circ} \mathrm{C}$ for 2 min, initial activation step $95^{\circ} \mathrm{C}$ for $15 \mathrm{~min}$ followed by 40 cycles of $95^{\circ} \mathrm{C}$ for 15 $\sec$ (denaturation), $\mathrm{x}^{\circ} \mathrm{C}$ for $30 \mathrm{sec}(\mathrm{x}=$ annealing temperature depending on the $\mathrm{Tm}$ of the used primers) and $72^{\circ} \mathrm{C}$ for $30 \mathrm{sec}$ (extension).

Primer sequences are provided in the oligos section. Mouse succinate dehydrogenase complex, subunit A (mSdha), was used as endogenous reference for normalization. For standard curves samples of undifferentiated or differentiated ESCs and maGSCs were used. Selection of the appropriate sample for the standard curve was based on preliminary experiments testing detection of expression of each gene by RT-PCR in the sample with the respective annealing temperature and for 35 cycles. As in miRNAs, specificity of the PCR products was confirmed with subsequent cloning and sequencing while reliability of realtime qRT-PCR data was also assessed in connection with the respective dissociation curves. 


\subsubsection{Data analysis}

Gene expression levels are calculated by determining the ratio between the amount of a target gene and an endogenous reference gene expressed in all samples. This ratio is then compared between different samples. Usually, housekeeping or maintenance genes are chosen as endogenous reference. The target and reference gene are amplified separately from the same sample.

The normalized value is determined for each sample and can be used to compare differential expression of a gene in different tissues. In this work relative quantification was carried out as follows:

- RNU6B (when testing miRNAs) or mSdha (when testing mRNAs). Their expression level does not change under the experimental conditions or between different tissues and they were therefore chosen as endogenous reference.

- A dilution series of a cDNA control sample (undifferentiated or differentiated ESC R1 or maGSC 129SV according to gene expression) was prepared to construct standard curves for target and reference genes.

- CT values for the standards and samples of interest were determined.

- Standard curves for both target and reference were constructed by plotting CT values ( $\mathrm{Y}$-axis) against the log of template amount or dilution (X-axis).

- Amount of target and reference in samples of interest were calculated using CT value and corresponding standard curve.

- The normalized amount of target was calculated by dividing the amount of target by the amount of reference (because replicate reactions were performed, average values were used).

- The normalized target amount of one sample was set to 1 (calibrator sample) and relative expression level of target gene in samples of interest was compared by dividing the normalized target amounts by the value of the calibrator. 


\subsection{Methods for subcloning and sequencing of RT-PCR products}

\subsubsection{Extraction of DNA from agarose gel}

In order to extract and purify DNA from agarose gels the QIAquick Gel Extraction Kit was used. With this method up to $400 \mathrm{mg}$ agarose can be processed per spin column. The principle of this method depends on selective binding of DNA to uniquely designed silica gel membranes.

Excised DNA fragments in agarose gel were isolated as described in QIAquick Spin Handbook supplied by manufacturer (Qiagen). In brief, to the excised DNA fragment from agarose gel, 3 volumes of QG buffer were added and then incubated at $50^{\circ} \mathrm{C}$ for $10 \mathrm{~min}$. For $>2 \%$ agarose gels, 6 volumes of QG buffer were added. After the gel slice was dissolved completely, 1 of isopropanol equal with the gel volume was added to the sample, which was then applied to a QIAquick column placed in a $2 \mathrm{ml}$ collection tube. The column was centrifuged for $1 \mathrm{~min}$. The flow-through was discarded and the column was washed twice with PE buffer with centrifugation for 1 min. After drying the column by additional centrifugation, it was placed into a fresh microcentrifuge tube. To elute DNA, $50 \mu \mathrm{l}$ of EB buffer was applied to the centre of the QIAquick membrane and centrifuged.

\subsubsection{Ligation in pGEM-T Easy Vector}

Taq and other polymerases have a terminal transferase activity that results in the non-template addition of a single nucleotide to the $3^{\prime}$ ends of PCR products. In the presence of all 4 dNTPs, dATP is preferentially added. This terminal transferase activity is the basis of the TA - cloning strategy. For cloning of these 
PCR products, the pGEM®T-easy Vector system that has $5^{\circ} \mathrm{DT}$ overhangs was used. The following constituents were mixed: $50 \mathrm{ng}$ of pGEM-T Easy vector PCR product (1:3 vector to insert ratio), $1 \mu \mathrm{T} 4$ DNA Ligase 10x buffer and 1 $\mu 1 \mathrm{~T} 4 \mathrm{DNA}$ Ligase in a total volume of $10 \mu \mathrm{l}$. The content was mixed by pipetting and the reaction was incubated at room temperature for $3 \mathrm{hrs}$.

\subsubsection{Transformation of DH5a competent bacteria}

Transformation of the bacteria was done by gently mixing one aliquot of competent bacteria DH5a $(50 \mu \mathrm{l})$ with $10 \mu \mathrm{l}$ of ligation reaction. After incubation for $30 \mathrm{~min}$ on ice, bacteria were heat shocked for $45 \mathrm{sec}$ at $42^{\circ} \mathrm{C}$ and cooled down for $5 \mathrm{~min}$ on ice. After adding $950 \mu 1$ of S.O.C. medium, bacteria were incubated at $37^{\circ} \mathrm{C}$ with shaking for $1 \mathrm{hr}$ to allow recovery of heat shocked bacteria. The bacteria were collected by centrifugation in $12000 \mathrm{x} \mathrm{rpm}$ for $30 \mathrm{sec}$ and the pellet was then plated out on LB-agar plates containing ampicillin $(50 \mu \mathrm{g} / \mathrm{ml})$ and whenever required $1 \mathrm{mM}$ IPTG and X-Gal $40 \mathrm{mg} / \mathrm{ml}$ were added for "Blue-White" selection. The LB-agar plates were incubated overnight at $37^{\circ} \mathrm{C}$.

\subsubsection{Cloning}

A single Escherichia coli colony was inoculated in $5 \mathrm{ml}$ of LB medium with ampicillin and incubated in a shaker for $16 \mathrm{hrs}$ at $37^{\circ} \mathrm{C}$ with a speed of $160 \mathrm{x}$ rpm. 


\subsubsection{Small-scale isolation of plasmid DNA (Mini preparation)}

One $\mathrm{ml}$ of the above mentioned saturated culture was used for making glycerol stocks and the rest of the culture was centrifuged at $4000 \mathrm{x}$ g for $10 \mathrm{~min}$ at $4^{\circ} \mathrm{C}$. Bacterial glycerol stocks were made suspending $600 \mu \mathrm{l}$ bacteria in $200 \mu \mathrm{l} 80 \%$ $(\mathrm{v} / \mathrm{v})$ sterile glycerol. The suspension was mixed well and stored at $-80^{\circ} \mathrm{C}$. The pellet was resuspended by vortexing in $100 \mu \mathrm{l}$ of solution P1. The bacterial cells were lysed with $200 \mu 1$ of P2 solution and then, after incubation for $5 \mathrm{~min}$ at room temperature, neutralized with $150 \mu$ of solution P3. The precipitated solution was centrifuged at $13000 \mathrm{x} \mathrm{g}$ for $20 \mathrm{~min}$. The supernatant was transferred into a new tube and centrifuged again for $10 \mathrm{~min}$ at $13000 \mathrm{x} \mathrm{g}$. The supernatant was transferred into a new tube, to this an equal volume of isopropanol was added and the tube was incubated on ice for $30 \mathrm{~min}$ and then centrifuged for $30 \mathrm{~min}$ at $13000 \mathrm{x}$ g at $4^{\circ} \mathrm{C}$. Finally the pellet was washed with $70 \%$ ethanol and centrifuged for $15 \mathrm{~min}$ at $13000 \mathrm{x} \mathrm{g}$ at $4{ }^{\circ} \mathrm{C}$. After air dried it was dissolved in $50 \mu 1$ of TE buffer.

\subsubsection{Digestion}

Successful ligation and cloning was confirmed through digestion of the plasmid DNA with the restriction enzyme recommended from pGEM®T-easy manufacturer, EcoRI. Restriction enzymes are class of bacterial enzymes that cut DNA at specific sites. Restriction enzyme digestion was performed by incubating the plasmid DNA with the appropriate amount of restriction enzyme in its respective buffer at $37^{\circ} \mathrm{C}$ for $1 \mathrm{~h}$ as recommended by the supplier. 


\subsubsection{DNA purification}

For successful downstream applications, isolated plasmid DNA should be of high purity. To clean the plasmid DNA from undesired byproducts of previous isolation processes, the Montage PCR Kit was used, following the instructions recommended by the supplier.

\subsubsection{Sequencing}

The non-radioactive sequencing was achieved with DYEnamic ET-Terminator Cycle Sequencing Kit. The principle of this is based on the Sanger method (Sanger and Coulson 1975). The sequencing reaction was carried out in a total volume of $10 \mu \mathrm{l}$ containing $1 \mu \mathrm{g}$ plasmid DNA or 100-200 ng purified PCR products, 5 pmol primer and $4 \mu$ reaction mix (contains dNTPs, dideoxy dye terminators and Taq DNA polymerase). Elongation and chain termination take place during the following program in a thermocycler: 5 min denaturing in $95^{\circ} \mathrm{C}$ followed by 25 cycles: $95^{\circ} \mathrm{C}, 30 \mathrm{sec}$ (denaturing); $55^{\circ} \mathrm{C}, 30 \mathrm{sec}$ (annealing); $60^{\circ} \mathrm{C}, 2 \mathrm{~min}$ (elongation). Then a final extension step followed at $72^{\circ} \mathrm{C}$ for $5 \mathrm{~min}$ and then a step at $4{ }^{\circ} \mathrm{C}$ for infinite time until the PCR tubes were taken from the PCR machine. After the sequencing reaction, the reaction products were analyzed with automatic sequencing equipment, namely MEGA BACE 1000. 


\subsection{Bioinformatics methods and software}

\subsubsection{Statistical analysis of data}

For statistical analysis of data, the STATISTICA software was used. Data are expressed as the mean $\pm \mathrm{SD}$. A one-way analysis of variance (ANOVA) followed by Fischer LSD's multiple comparison tests was used for statistical analysis with $\mathrm{P}$ less than 0.05 considered statistically significant.

\subsection{2 miRNA target prediction}

For prediction of miRNA targeting sites in mRNAs 3'UTRs the TARGETSCAN Software Release 4.2 was used (http://www.targetscan.org/vert_42/)).

\subsubsection{Sanger miRNA database (miRBase)}

All miRNA sequences were provided by or correlated with the sequences stored in the Sanger miRNA data base (http://microrna.sanger.ac.uk).

\subsubsection{Blast}

The obtained DNA sequences were analyzed with a BLAST program by using the sequence data base Genbank (www.ncbi.nlm.nih.gov). 


\subsubsection{SDS software}

Visualization and primary raw data processing of the real-time qRT-PCR results was performed by the SDS Software (Version 2.1).

\subsubsection{Microsoft Excel}

Processing of the real-time qRT-PCR raw data and visualization of the results was done using the Microsoft Office Excel Software. 


\section{RESULTS}

\section{1 miRNA array expression profiling of ESCs and maGSCs during differentiation}

In a previous study conducted in the Institute of Human Genetics, Goettingen, the miRNA profile of undifferentiated ESCs and maGSCs has been examined in order to compare these two cell types (Zovoilis 2009). Aim of the present study was to investigate the miRNA expression profile of ESCs and maGSCs during differentiation by analyzing differences in levels of miRNAs between differentiated and the respective undifferentiated cells.

To this purpose differentiated ESCs and maGSCs and the respective undifferentiated cells were included in a global miRNA array. In detail, RNA from the following cells was included in the array:

- differentiated ESCs of the ESC R1 cell line (ESCs_Diff) and undifferentiated ESCs of the same cell line (ESCs_Undiff),

- differentiated maGSCs of the maGSC 129SV cell line (maGSCs_Diff) and undifferentiated maGSCs of the same cell line (maGSCs_Undiff).

Both ESC R1 and maGSC 129SV have been derived from 129/Sv mice.

Differentiation was performed by treatment for 21 days in gelatine coated flasks in the absence of feeder layer and LIF and in the presence of retinoic acid (RA), an inducer of differentiation (Fig. 3). 


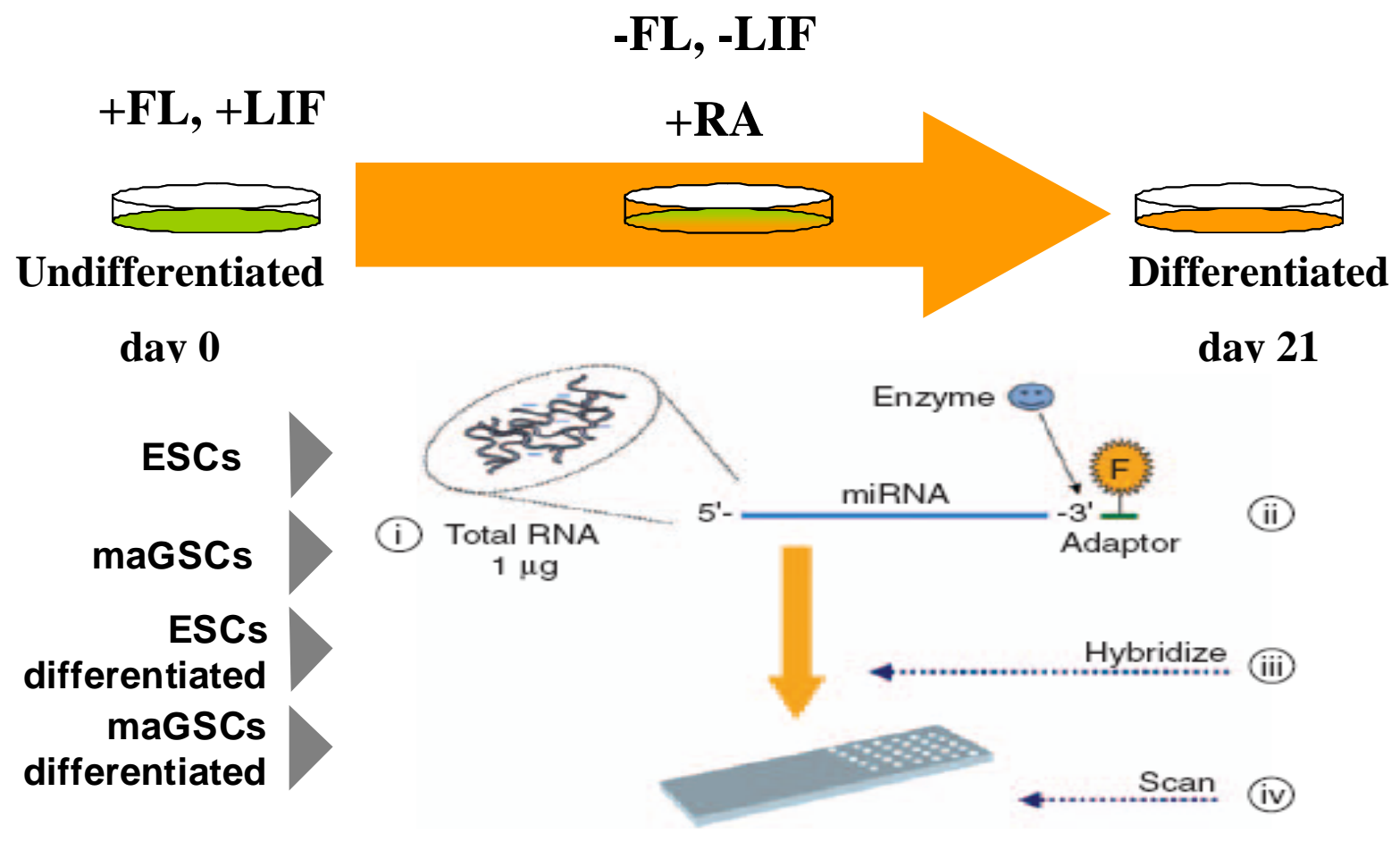

Figure 3. miRNA array expression profiling of ESCs and maGSCS during differentiation. (A) Differentiation of ESCs and maGSCs was performed for 21 days in gelatine coated flasks, without feeder layer (FL) and LIF but with RA. The array tested the miRNA expression between undifferentiated state (undifferentiated-day 0) and after 21 days of differentiation (Differentiated-day 21). (B) Total RNA prepared with a method that preserves small RNA species was extracted from the cells and miRNAs were labeled enzymatically with a fluorescent molecule to their 3'end. The labeled miRNAs were hybridized on slides containing capture probes complementary to mature miRNAs registered in miRBase release 11.0 and the slides were finally scanned (Source: www.exiqon.com/array).

The comparisons between the four samples were carried out according to the experimental layout shown in figure 4 (each arrow depicts two hybridizations, each time with two different labelling colours, as described in Materials and Methods). 


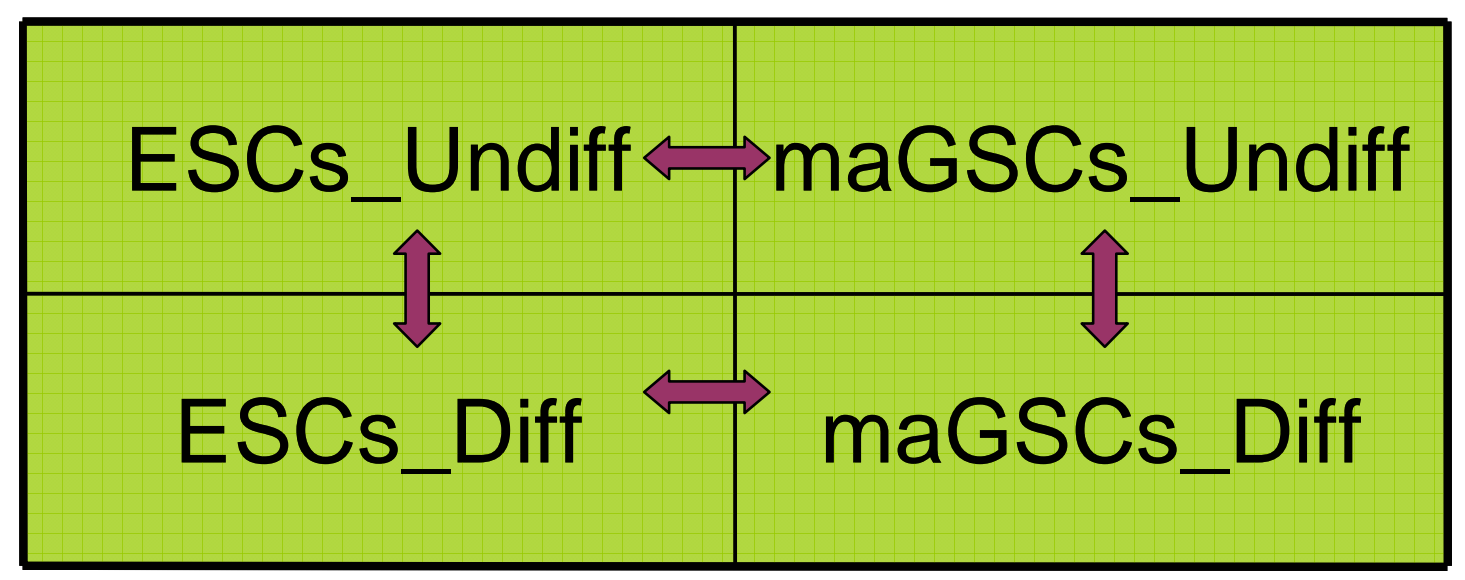

Figure 4. Experimental layout of miRNA array expression profiling of ESCs and maGSCs during differentiation. Each arrow represents two hybridizations (two identical slides for each comparison/hybridization, eight in total, see also Materials and Methods).

Tables 1 and 2 demonstrate the results obtained by the array.

These tables consist of:

i) differences in miRNA expression observed between undifferentiated and differentiated cells that were statistically significant and

ii) differences in expression of miRNAs that have been shown to be pluripotency-related. These are members of miR-290 cluster (Houbaviy et al. 2003), miR-302 cluster (Strauss et al. 2006) and miR17-92 cluster (Hayashi et al. 2007). 
3.1.1 Comparison between undifferentiated ESCs (ESCs_Undiff) and differentiated ESCs (ESCs_Diff) after a 21-day treatment under differentiation conditions

$\left.\begin{array}{|c|c|}\hline \text { miRNAs } & \text { ESCs Undiff-ESCs Diff } \\
\hline \text { mmu-miR-290 } & 2.5 \\
\hline \text { mmu-miR-291 } & 1.2 \\
\hline \text { mmu-miR-292 } & -0.48 \\
\hline \text { mmu-miR-293 } & 2 . *^{*} \\
\hline \text { mmu-miR-294 } & 1.9 \\
\hline \text { mmu-miR-295 } & 1.7 \\
\hline \text { mmu-miR-302a } & -0.4 \\
\hline \text { mmu-miR-302b } & 0 \\
\hline \text { mmu-miR-302c } & -0.3 \\
\hline \text { mmu-miR-302d } & 0.4 \\
\hline \text { mmu-miR-17 } & 2.3^{*} \\
\hline \text { mmu-miR-18a } & 0.2 \\
\hline \text { mmu-miR-19a } & 1.5 \\
\hline \text { mmu-miR-19b } & 1.8 \\
\hline \text { mmu-miR-20a } & 2.5^{*} \\
\hline \text { mmu-miR-92a } & 0.6 \\
\hline \text { mmu-miR-711 } & 1.5 \\
\hline \text { mmu-miR-320 } & 2.0^{*} \\
\hline \text { mmu-miR-720 } & 2.1 * \\
\hline \text { miR-290 cluster } \\
\hline \text { miR-302 cluster } \\
\hline \\
\text { miR-17-92 } \\
\text { cluster } \\
\hline \\
\text { other miRNAs } \\
\hline\end{array}\right\}$\begin{tabular}{c} 
\\
\hline \\
\hline
\end{tabular}

Table 1. Differentially expressed miRNAs between undifferentiated ESCs (ESCs_Undiff) and differentiated ESCs (ESCs_Diff). ESCs were cultured 21 days on gelatine, without feeder layer and LIF but with RA. The * indicates values that are statistically significant. Value numbers $(\mathrm{x})$ show differential expression $2^{\wedge} \mathrm{x}$ times. Positive values show ESCs_Undiff $>$ ESCs_Diff, while negative values show the opposite. 
In detail, five miRNAs are significantly reduced in differentiated ESCs in comparison to the undifferentiated ESCs (marked with * in Table 1) and are the following:

- miR-293, a member of miR-290 cluster,

- $\operatorname{miR}-17$ and miR-20a, members of miR-17-92 cluster,

- $\operatorname{miR}-720$ and

- $\operatorname{miR}-320$.

No change concerning the levels of members of miR-302 cluster, as well as miR-711, was observed in the comparison between ESCs_Undiff and ESCs_Diff. 
3.1.2 Comparison between undifferentiated maGSCs (maGSCs_Undiff) and differentiated maGSCs (maGSCs_Diff) after a 21-day treatment under differentiation conditions

\begin{tabular}{|c|c|c|}
\hline miRNAs & maGSCs_Undiff-maGSCs_Diff & \\
\hline mmu-miR-290 & 4.1 & \\
\hline mmu-miR-291 & $2.2^{*}$ & \\
\hline mmu-miR-292 & $2.3^{*}$ & \multirow{2}{*}{$\begin{array}{c}\text { miR-290 } \\
\text { cluster }\end{array}$} \\
\hline mmu-miR-293 & $4.6^{*}$ & \\
\hline mmu-miR-294 & 3.0 & \multirow{5}{*}{$\begin{array}{c}\text { miR-302 } \\
\text { cluster }\end{array}$} \\
\hline mmu-miR-295 & $6.2^{*}$ & \\
\hline mmu-miR-302a & -0.2 & \\
\hline mmu-miR-302b & 0 & \\
\hline mmu-miR-302c & -1.1 & \\
\hline mmu-miR-302d & 0 & \multirow{5}{*}{$\begin{array}{c}\text { miR-17-92 } \\
\text { cluster }\end{array}$} \\
\hline mmu-miR-17 & 1.1 & \\
\hline mmu-miR-18a & -0.1 & \\
\hline mmu-miR-19a & 0 & \\
\hline mmu-miR-19b & 0.8 & \\
\hline mmu-miR-20a & 1.4 & \\
\hline mmu-miR-92a & 0.7 & \\
\hline mmu-miR-320 & 0.7 & \multirow{3}{*}{$\begin{array}{c}\text { other } \\
\text { miRNAs }\end{array}$} \\
\hline mmu-miR-720 & 2.3 & \\
\hline mmu-miR-711 & $2.7^{*}$ & \\
\hline
\end{tabular}

Table 2. Differentially expressed miRNAs between undifferentiated maGSCs (maGSCs_Undiff) and differentiated maGSCs. maGSCs were cultured 21 days on gelatine, without feeder layer and LIF but with RA (maGSCs_Diff). The * indicates values that are statistically significant. Value numbers $(x)$ show differential expression $2^{\wedge} x$ times. Positive values show maGSCs_Undiff $>$ maGSCs_Diff, while negative values show the opposite. 
In detail, five miRNAs are significantly reduced in differentiated maGSCs as compared to undifferentiated maGSCs (marked with * in the table above) and are the following:

- miR-291, miR-292, miR-293, miR-295, all of which are members of miR290 cluster, and

- $\operatorname{miR}-711$.

In contrast to ESCs, miR-17-92 cluster did not differ in the comparison between undifferentiated and differentiated maGSCs. Also miR-720 and miR-320 showed no change in this comparison.

\subsubsection{Validation of the array data by real-time qRT-PCR}

miRNA array profiling allows large scale screening of miRNA expression. In order to validate the reliability of the array results, an independent and highly sensitive method, that of real-time qRT-PCR, was used. The validation of the array data included the miRNAs that differed significantly in at least one of the comparisons shown in tables 1 and 2 (this between ESCs_Undiff and ESCs_Diff and that between maGSCs_Undiff and maGSCs_Diff). These are members of miR-290 cluster, members of miR-17-92 cluster and other miRNAs, such as miR-720, miR-711 and miR-320. 


\subsubsection{1 miR-290 cluster}

Most of the miRNAs that differed in the comparisons of ESCs_Undiff with ESCs_Diff and of maGSCs_Undiff with maGSCs_Diff belong to the miR-290 cluster. To validate these data qRT-PCR was performed for all six members of the cluster (including miR-291, 292, 293 and 295, which differed in the array, and miR-290 and miR-294, which showed no statistical significant difference in the array data). The qRT-PCR showed significant downregulation of all members of the cluster in both pluripotent cell lines during differentiation (Fig. $5)$.

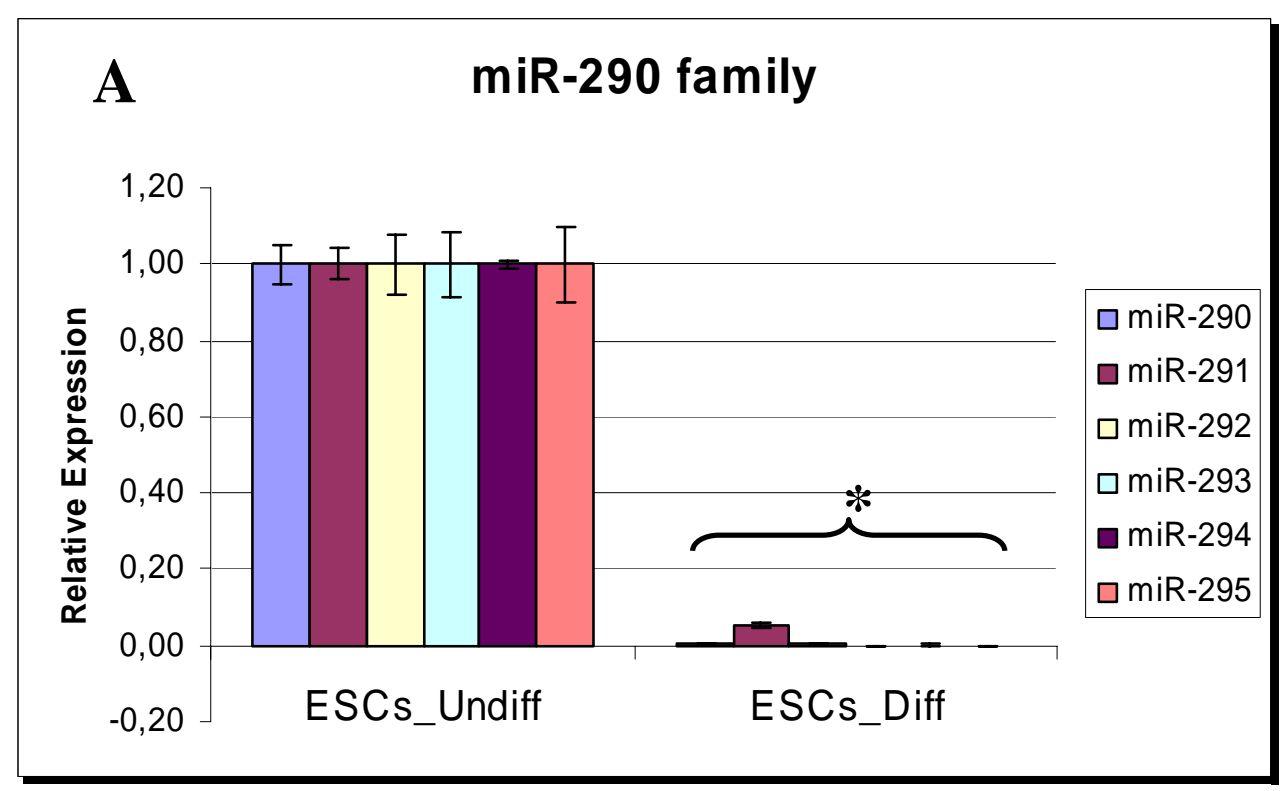




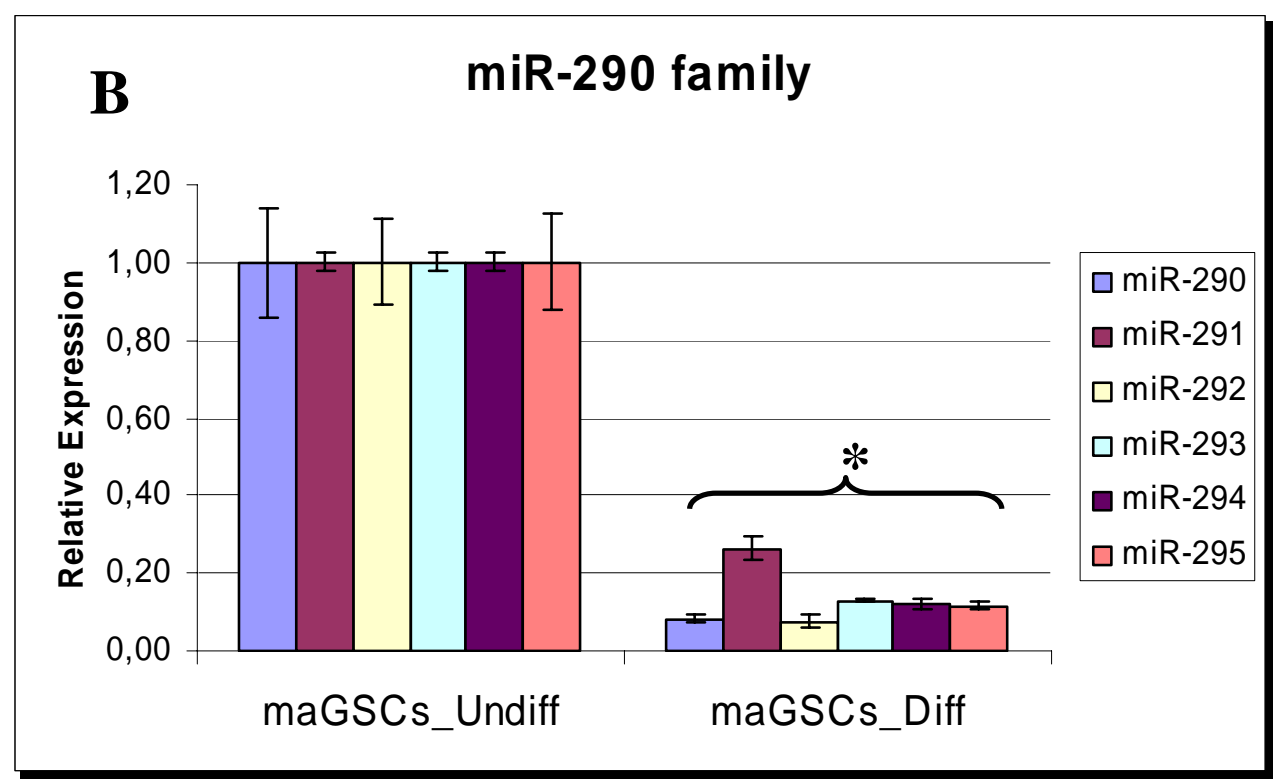

Figure 5. Validation of miR-290 cluster by real-time qRT-PCR. miRNA expression levels of miR-290 cluster were determined by real-time qRT-PCR A: in undifferentiated ESCs (ESCs_Undiff) and differentiated ESCs after 21-day treatment on gelatine without feeder layer and LIF but with RA (ESCs_Diff), B: in undifferentiated maGCSs (maGSCs_Undiff) and differentiated maGSCs after 21-day treatment on gelatine without feeder layer and LIF but with RA (maGSCs_Diff). Expression levels were normalized to the level of the endogenous control (RNU6B). For every miRNA, ESCs_Undiff and maGSCs_Undiff were respectively used as calibrators, error bars indicate standard deviation. Asterisks indicate statistical significance for the difference between differentiated and the respective undifferentiated cells. 


\subsubsection{2 miR-17-92 cluster}

Two members of miR-17-92 cluster, miR-17 and miR-20a, displayed a significant difference in the comparison ESCs_Undiff-ESCs_Diff. This difference could not be observed in the comparison maGSCs_UndiffmaGSCs_Diff. The qRT-PCR confirmed the array results for both miRNAs (Fig. 6).
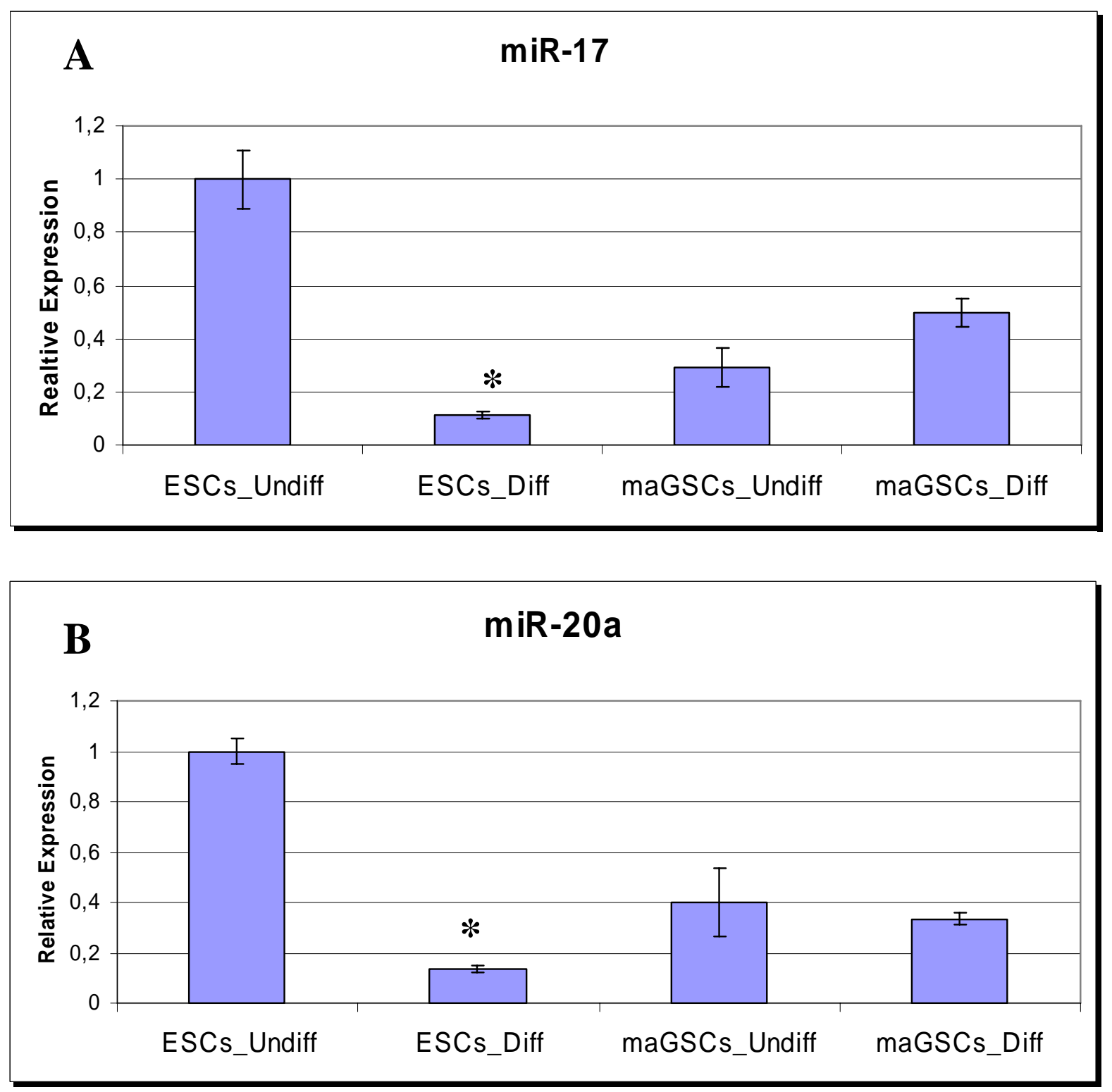
Figure 6. Validation of $\mathrm{miR}-17$ and miR-20a by real-time qRT-PCR. miRNA expression levels of miR-17 (A) and miR-20a (B) were determined by real-time qRT-PCR in undifferentiated cells and in differentiated cells after 21day treatment on gelatine without feeder layer and LIF but with RA (ESCs_Undiff/ESCs_Diff and maGSCS_Undiff/maGSCs_Diff). Expression levels were normalized to the level of the endogenous control (RNU6B). For both miRNAs ESCs_Undiff were used as calibrator, error bars indicate standard deviation. Asterisks indicate statistical significance for the difference between differentiated and the respective undifferentiated cells.

\subsubsection{3 miR-720}

According to the qRT-PCR results, levels of miR-720 did not differ significantly between undifferentiated and differentiated ESCs and between undifferentiated and differentiated maGSCs. The array data could not be confirmed (Fig. 7).

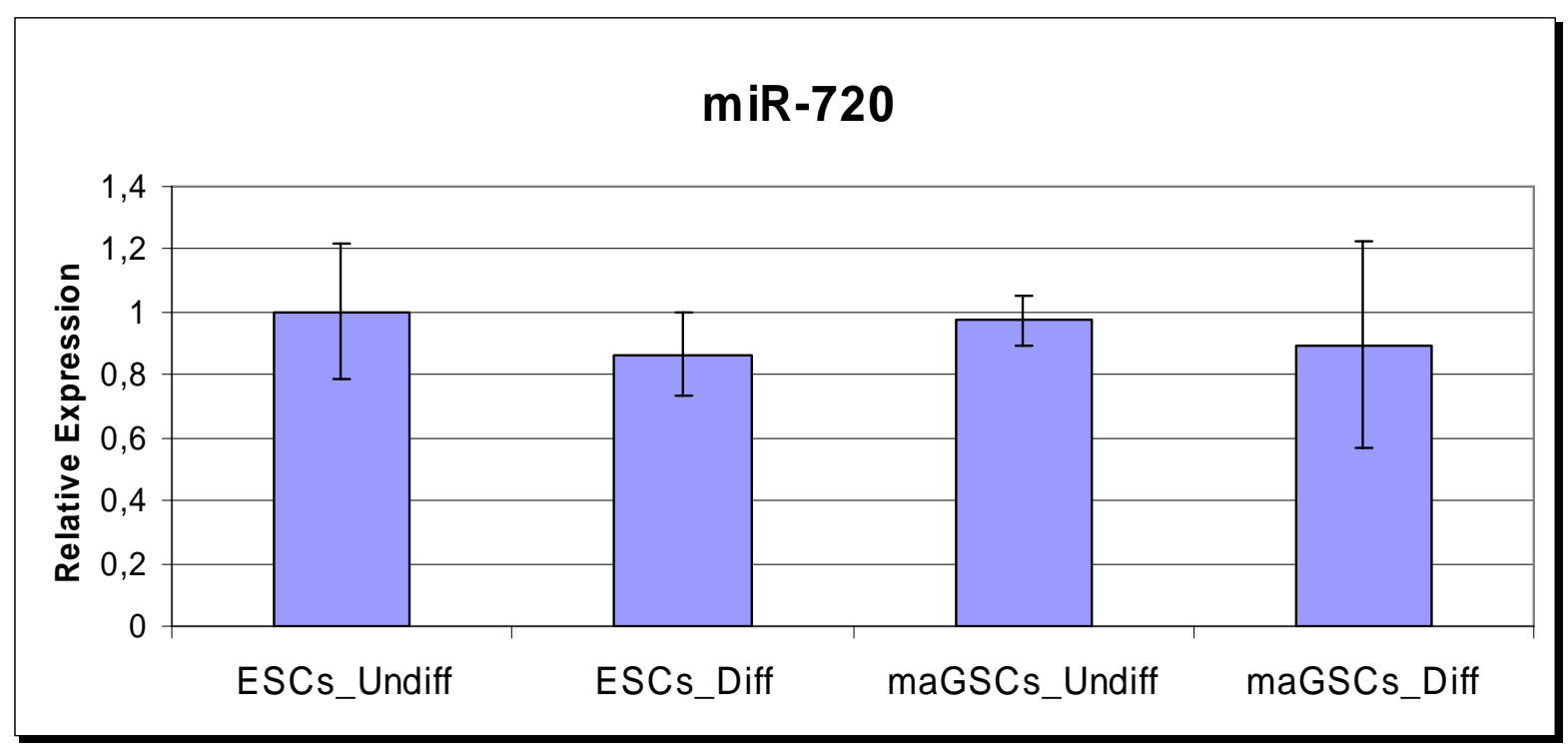


Figure 7. Validation of miR-720 by real-time qRT-PCR. miRNA expression levels of miR-720 were determined by real-time qRT-PCR in undifferentiated cells and in differentiated cells after 21-day treatment on gelatine without feeder layer and LIF but with RA (ESCs_Undiff/ESCs_Diff and maGSCS_Undiff/maGSCs_Diff). Expression levels were normalized to the level of the endogenous control (RNU6B). ESCs_Undiff were used as calibrator, error bars indicate standard deviation.

\subsubsection{4 miR-711}

Regarding miR-711, no expression of this miRNA could be detected in undifferentiated and differentiated cells of both lines. qRT-PCR amplified various unspecific products and failed to verify any of the array data, suggesting that miR-711 may not be expressed in ESCs and maGSCs.

\subsubsection{3 miR-320}

miR-320 displayed a 50\% reduction of its expression level determined by qRTPCR during differentiation in ESCs (comparison between ESCs_Undiff and ESCs_Diff)-although the array data suggest that this difference should be 4 times high. In contrast, after differentiation of maGSCs (comparison between maGSCs_Undiff and maGSCs_Diff), qRT-PCR revealed a 2-time increase in the levels of miR-320, which is in contradiction to the array data that exhibit no significant difference (Fig. 8). 


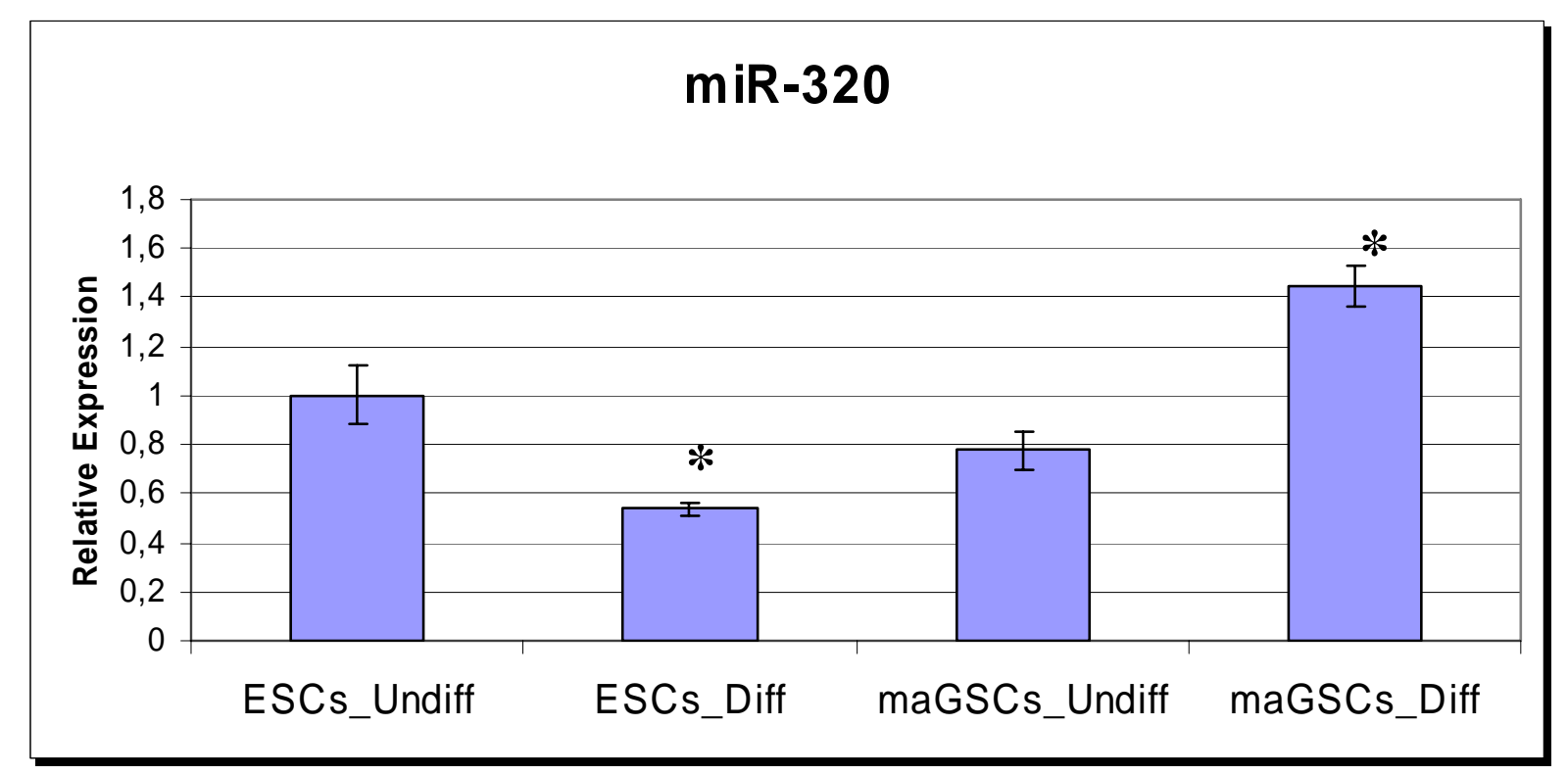

Figure 8. Validation of miR-320 by real-time qRT-PCR. miRNA expression levels of miR-320 were determined by real-time qRT-PCR PCR in undifferentiated cells and in differentiated cells after 21-day treatment on gelatine without feeder layer and LIF but with RA (ESCs_Undiff/ESCs_Diff and maGSCS_Undiff/maGSCs_Diff). Expression levels were normalized to the level of the endogenous control (RNU6B). ESCs_Undiff were used as calibrator, error bars indicate standard deviation. Asterisks indicate statistical significance for the difference between differentiated and the respective undifferentiated cells. 


\subsection{Expression profiling of members of miR-17-92 cluster during differentiation in ESCs and maGSCs}

miR-17-92 cluster is known to play an important role in embryonic development and to be connected to cell proliferation (Hayashi et al. 2008). According to the array data, this cluster is downregulated during differentiation of ESCs, but not of maGSCs.

The miRNA array profiling included only one differentiation condition (without feeder layer and LIF but with RA, "Gel+RA" condition) and only one time point (day 21). In order to examine in detail the expression profile of miR-17-92 cluster during differentiation, the study of ESCs and maGSCs was expanded to an additional differentiation condition (without feeder layer and LIF and without RA, "Gel" condition) and to more time points (day 5 and day 10). ESCs of the ESC R1 cell line and maGSCs of the maGSC 129SV cell line were cultured on gelatine, without feeder layer (FL) and LIF, but in the presence or absence of RA, as shown in figure 9. 


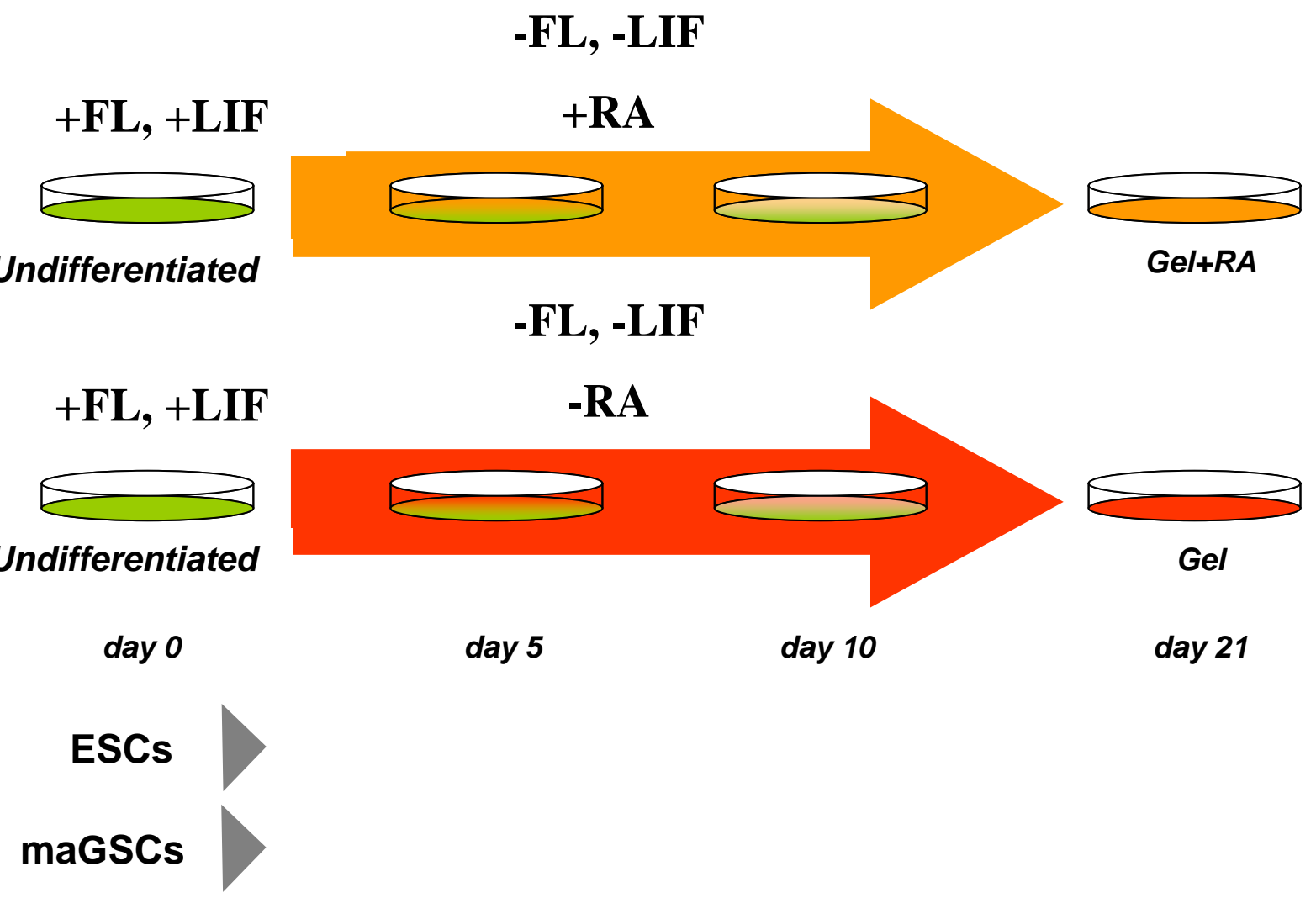

Figure 9. Differentiation of ESCs and maGSCs. The differentiation of both cell types was performed for 21 days in gelatine coated flasks without feeder layer (FL) and LIF, but with/without RA. Cells were tested in undifferentiated state (undifferentiated-day 0) and after 5, 10 and 21 days of differentiation (day 5 , day 10 and day 21$)$.

Two representative members of the miR-17-92 cluster (miR-17 and miR-20a) as well as miR-19b and miR-20b, which are two members of a paralog of miR-1792 cluster, miR-106a-363 cluster, were tested after 5, 10 and 21 days of differentiation.

Day 0 in the diagram indicates undifferentiated cells that were subsequently used in the differentiation. The results for every miRNA are summarised, first for ESCs (Figures 10A, 11A, 12A, 13A) and then for maGSCs (Figures 10B, 11B, 12B, 13B). 

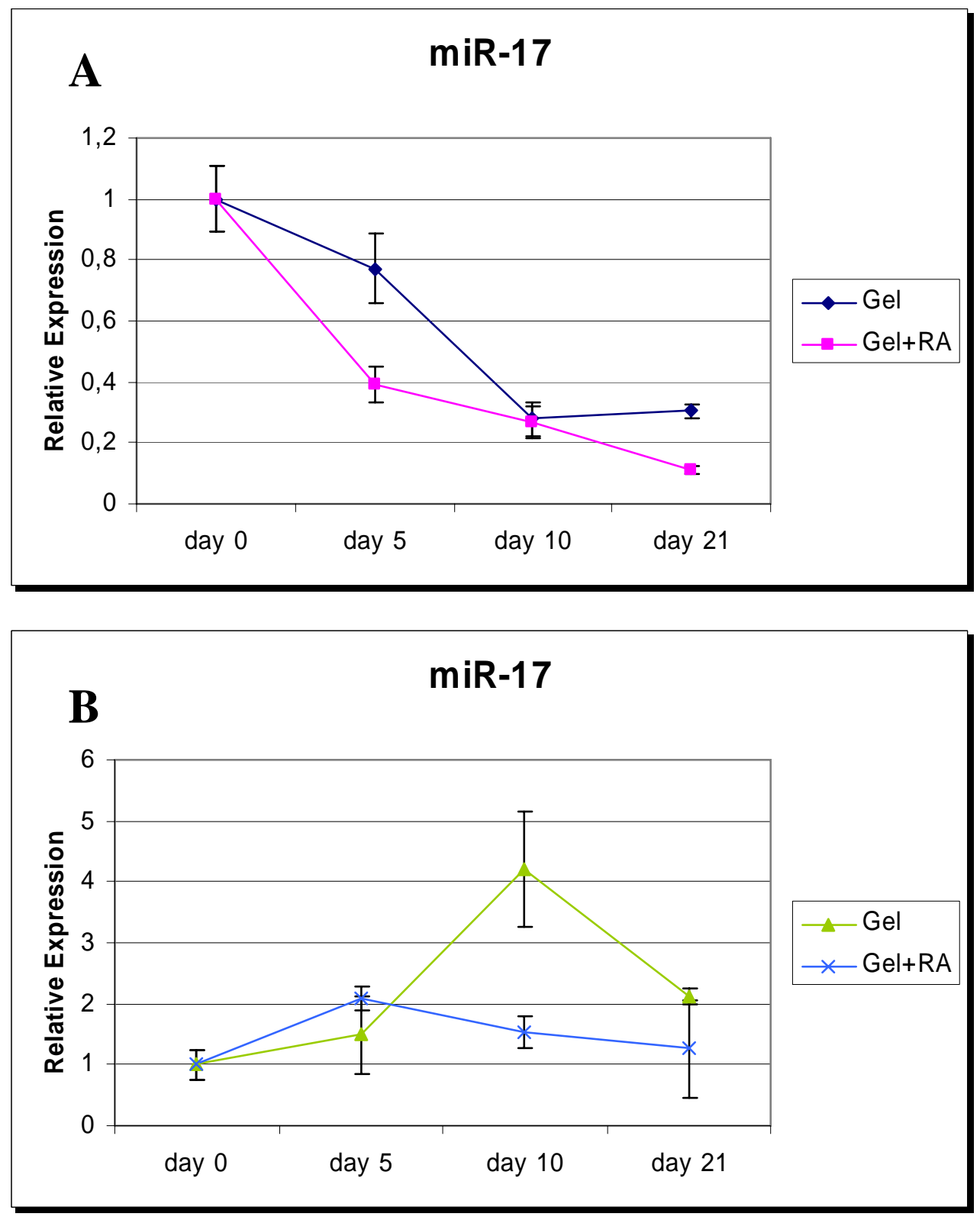

Figure 10. miR-17 during differentiation of ESCs and maGSCs. miRNA expression levels of miR-17 were determined by real-time qRT-PCR A: in undifferentiated ESCs (day 0) and in ESCs differentiated on gelatine, without feeder layer (FL) and LIF but with/without RA (Gel+RA/Gel), B: in undifferentiated maGSCs (day 0) and maGSCs differentiated as described for the ESCs. Expression levels were normalized to the level of the endogenous control (RNU6B). Day 0 was used as calibrator, error bars indicate standard deviation. 
Figure 10 shows a notable downregulation of miR-17 during differentiation of ESCs in both culture conditions. In the case of maGSCs, during differentiation under the gelatine and RA condition (Gel+RA) levels of miR-17 remain stable, whereas under the gelatine condition (Gel) miR-17 is significantly upregulated until day 10 and later downregulated until day 21.
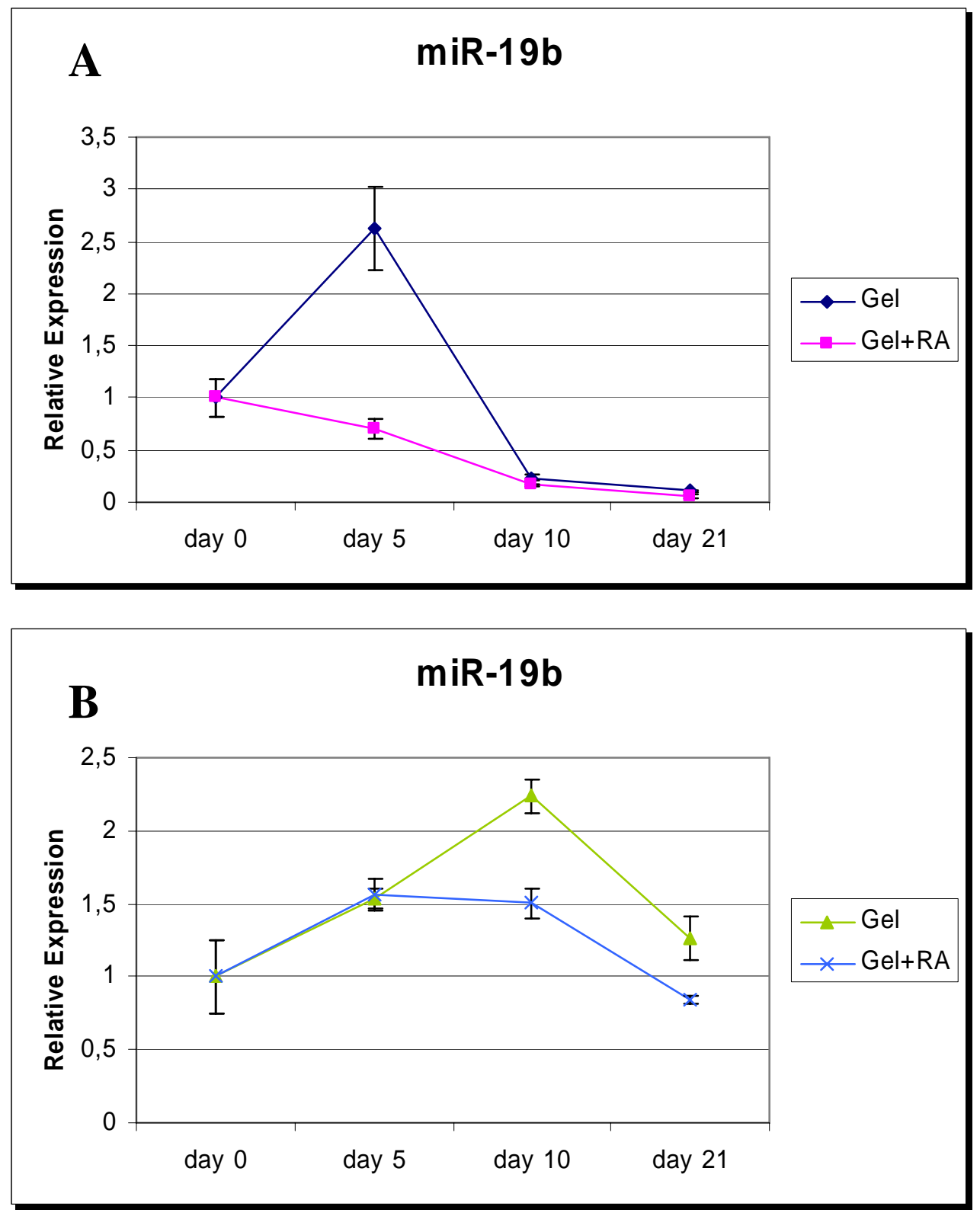

Figure 11. miR-19b during differentiation of ESCs and maGSCs. miRNA expression levels of miR-19b were determined by real-time qRT-PCR A: in undifferentiated ESCs (day 0) and in ESCs differentiated on gelatine, without 
feeder layer (FL) and LIF but with/without RA (Gel+RA/Gel), B: in undifferentiated maGSCs (day 0) and maGSCs differentiated as described for the ESCs. Expression levels were normalized to the level of the endogenous control (RNU6B). Day 0 was used as calibrator, error bars indicate standard deviation.

In ESCs miR-19 (Fig. 11) displays a different profile to this of miR-17 during differentiation, with a significant increase of its levels until day 5 followed by a decrease at day 10 and day 21 . In maGSCs miR-19 resembles miR-17 in both culture conditions.

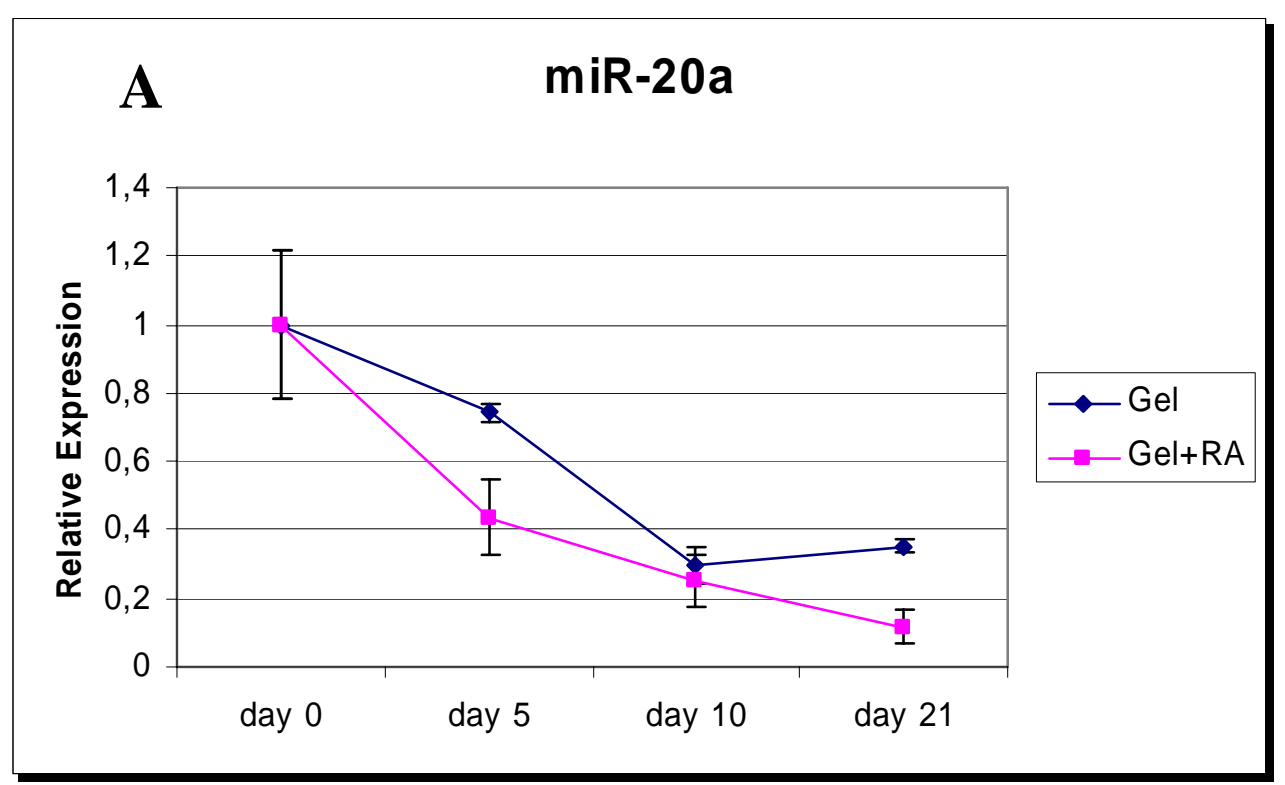




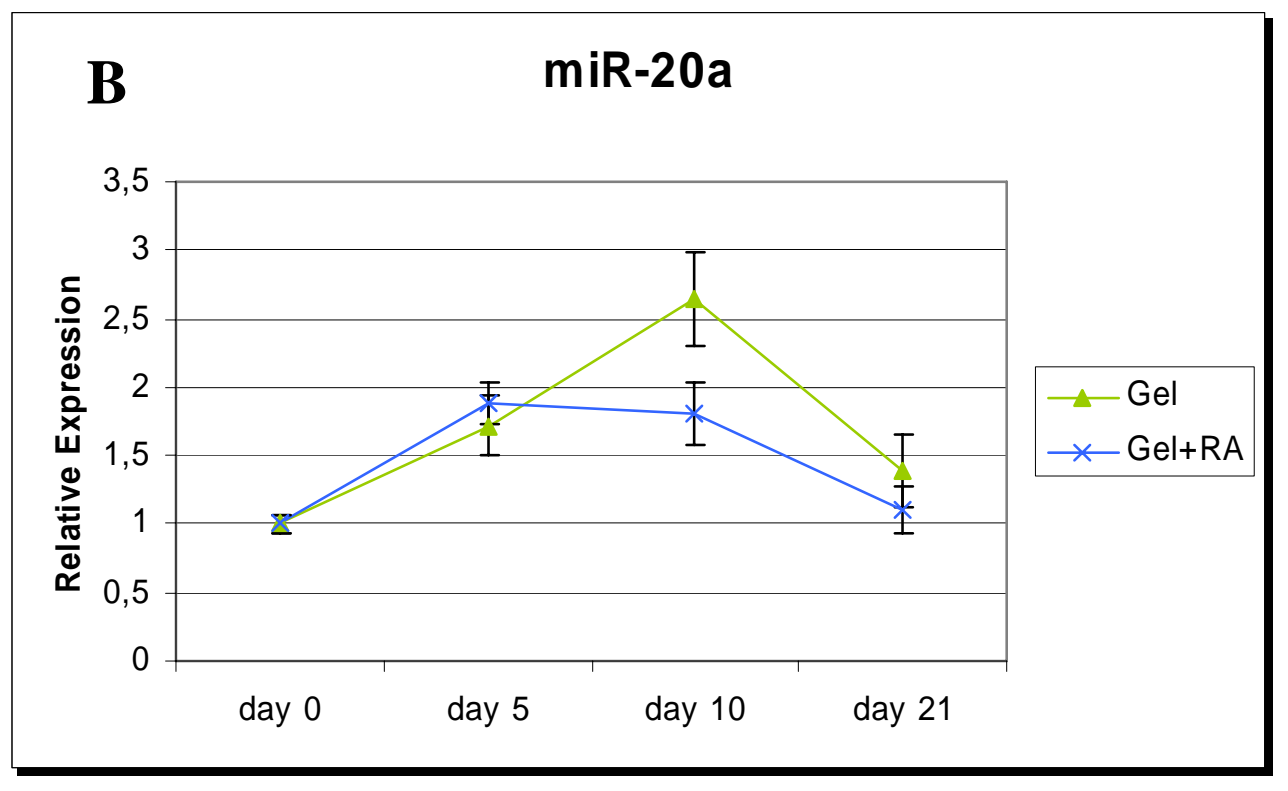

Figure 12. miR-20a during differentiation of ESCs and maGSCs. miRNA expression levels of miR-20a were determined by real-time qRT-PCR A: in undifferentiated ESCs (day 0) and in ESCs differentiated on gelatine, without feeder layer (FL) and LIF but with/without RA (Gel+RA/Gel), B: in undifferentiated maGSCs (day 0) and maGSCs differentiated as described for the ESCs. Expression levels were normalized to the level of the endogenous control (RNU6B). Day 0 was used as calibrator, error bars indicate standard deviation.

During differentiation of both ESCs and maGSCs miR-20a (Fig. 12) follows a similar pattern with miR-17. 

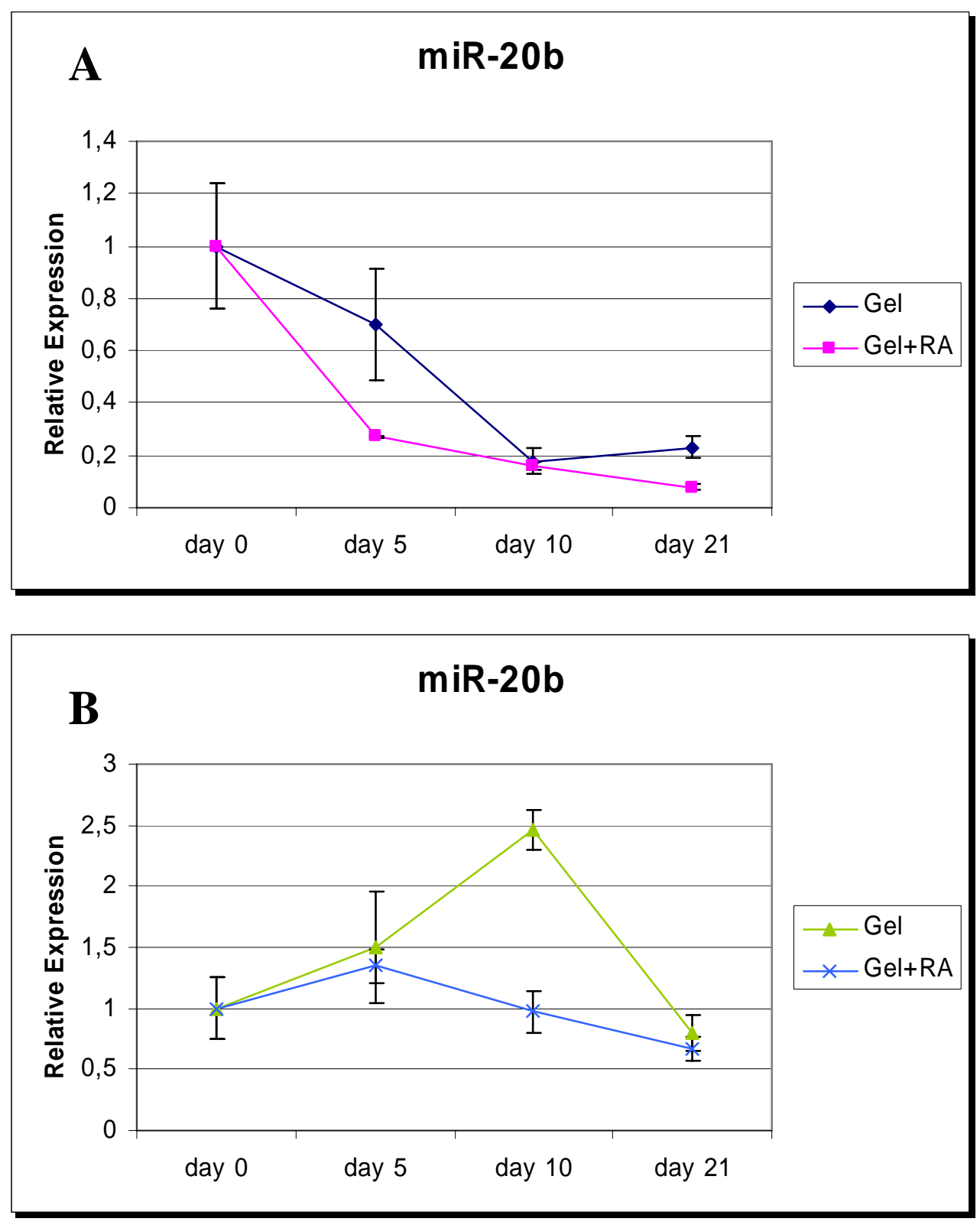

Figure 13. miR-20b during differentiation of ESCs and maGSCs. miRNA expression levels of miR-20b were determined by real-time qRT-PCR A: in undifferentiated ESCs (day 0) and in ESCs differentiated on gelatine, without feeder layer (FL) and LIF but with/without RA (Gel+RA/Gel), B: in undifferentiated maGSCs (day 0) and maGSCs differentiated as described for the ESCs. Expression levels were normalized to the level of the endogenous control (RNU6B). Day 0 was used as calibrator, error bars indicate standard deviation. 
The same applies for miR-20b (Fig. 13) for both cell types and culture conditions.

To summarize, in case of ESCs, all four miRNAs, miR-17, miR-19b, miR-20a and miR-20b, show a significant downregulation gradually until day 21, resulting in a $70-90 \%$ decrease in expression levels. With both culture conditions, gelatine and RA (Gel+RA) and gelatine but without RA (Gel), this downregulation is demonstrated, but it is stronger and earlier in the case of gelatine and RA condition. The only exception concerns miR-19b at day 5, where a 2.5-time upregulation is observed, which is followed by a remarkable reduction at day 10 and 21 .

As far as maGSCs are concerned, these four miRNAs remain in high levels during differentiation. Under gelatine and RA condition no significant change is seen during 21 days, whereas under gelatine condition an upregulation of the miRNAs marks day 10 and is followed until day 21 by downregulation to levels similar to those of day 0 (undifferentiated cells).

Comparing day 0 with day 21, ESCs demonstrate an important downregulation of miR-17 and miR-20a, while maGSCs show no difference for these two miRNAs. This is consistent with the array data and validates their accuracy. 


\subsection{Expression profiling of pluripotency-related miRNAs during differentiation of embryonic germ cells (EGCs)}

This study has so far concentrated on the miRNA profile during differentiation in two characteristic types of pluripotent cells, ESCs and maGSCs. Pluripotency-related miRNAs are also highly expressed in primordial germ cells (PGCs), which are germ cell precursors in the embryo, and play a pivotal role in their proliferation (Hayashi et al. 2008).

For this reason, this study was broadened to embryonic germ cells (EGCs), another pluripotent cell type that is derived from the PGCs. EGCs were cultured on gelatine without feeder layer (FL) and LIF, but in the presence or absence of RA, as shown in figure 14 .

\section{-FL, -LIF \\ $+\mathbf{F L},+\mathbf{L I F}$ \\ $+\mathbf{R A}$}

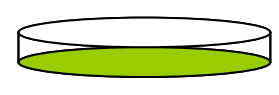

Undifferentiated

+FL, +LIF

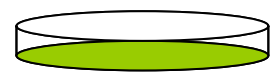

Undifferentiated

day 0

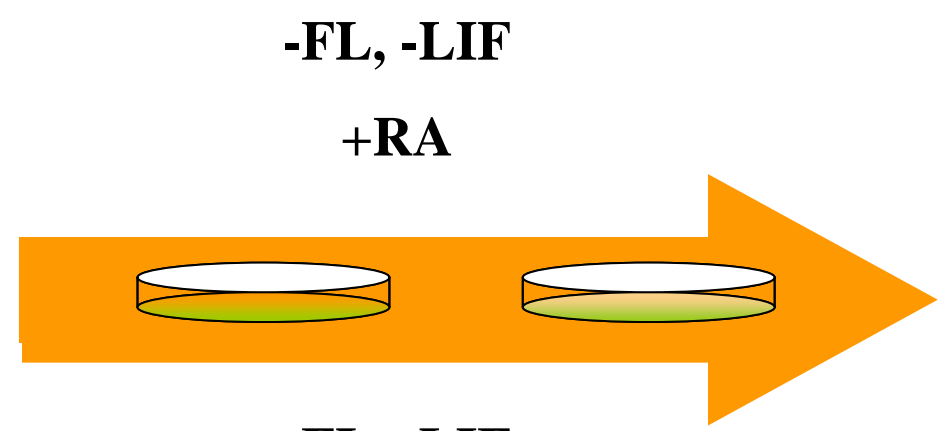

-FL, -LIF

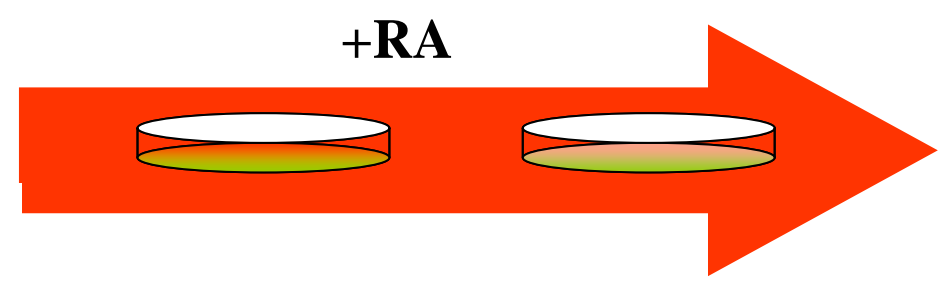

day 5

day 10

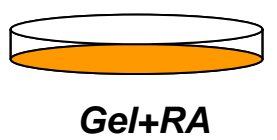

Gel+RA

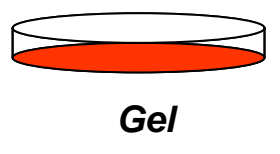

day 21

\section{EGCs}


Figure 14. Differentiation of EGCs. The differentiation of EGCs was performed for 21 days in gelatine coated flasks, without feeder layer (FL) and LIF, but with/without RA. Cells were tested in undifferentiated state (day 0 ) and after 5, 10 and 21 days of differentiation (day 5, day 10 and day 21).

EGCs were later tested with qRT-PCR on days 5, 10 and 21 for one member of miR-290 cluster, miR-290, one member of miR-302 cluster, miR-302a, and one member of miR-17-92 cluster, miR-17 (Fig. 15).
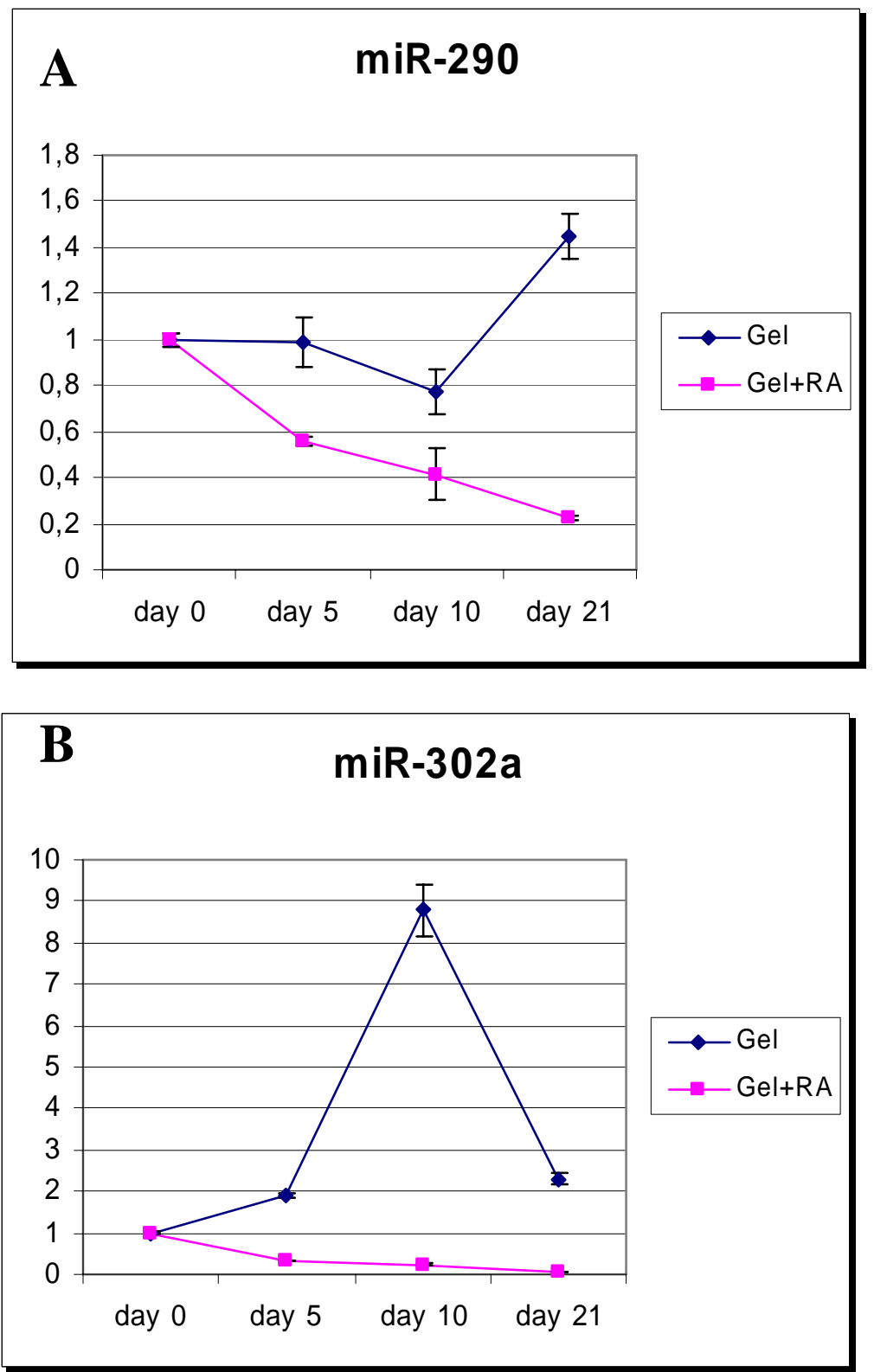


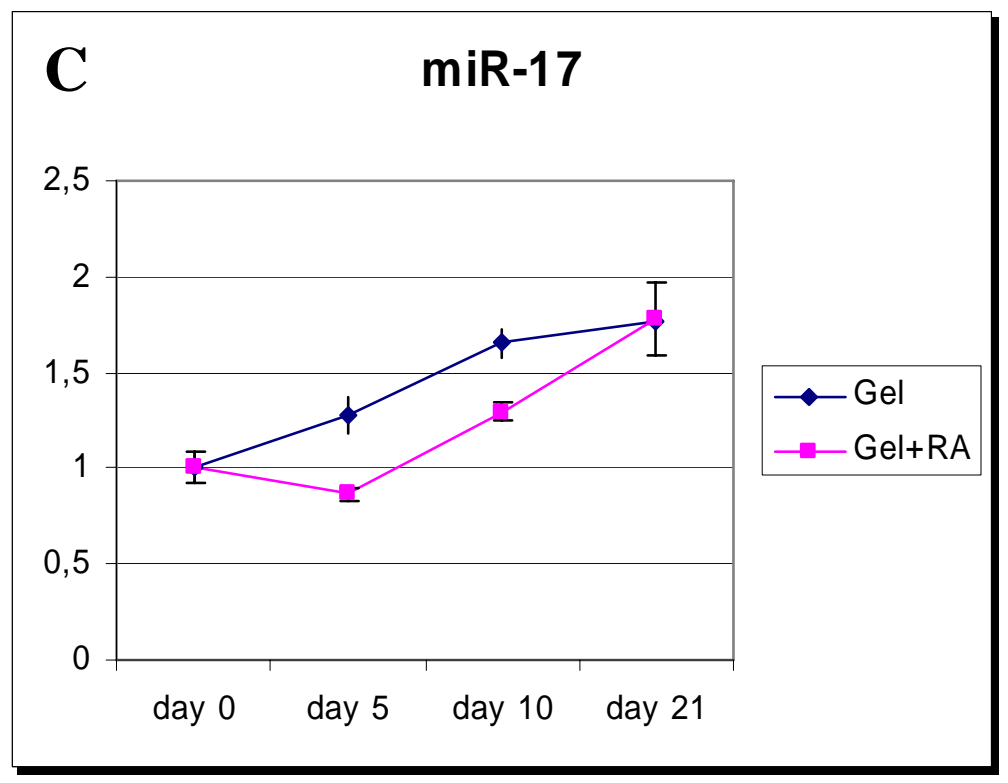

Figure 15. miR-290, miR-302a and miR-17 during differentiation of EGCs. miRNA expression levels of miR-290 (A), miR-302a (B) and miR-17 (C) were determined by real-time qRT-PCR in ESCs cultured on gelatine without feeder layer (FL) and LIF but with/without RA (Gel+RA/Gel), for 5, 10 and 21 days. Expression levels were normalized to the level of the endogenous control (RNU6B). Undifferentiated EGCs (day 0) were used as calibrator, error bars indicate standard deviation.

Figure 15 shows that miR-290 and miR-17 remain on high levels in EGCs that undergo differentiation under the gelatine (Gel) condition. Under the gelatine and RA (Gel+RA) culture condition, miR-290 is downregulated.

miR-302a reacts in a different manner than miR-290 and miR-17, as under Gel condition it increases between day 5 and day 10 and then it is downregulated. miR-302a under Gel+RA culture condition decreases gradually. 


\subsection{Functional studies of miR-302 cluster}

miR-302 cluster is considered to be pluripotency-related, as it is highly expressed in ESCs, maGSCs and, as shown in this study (Fig. 15B), also in EGCs; however they are downregulated during ESC differentiation and undetectable in adult mouse organs.. Nevertheless, its role in maintenance of pluripotency and in the process of differentiation is still unknown. In order to elucidate the function of miR-302 cluster, four members of it, miR-302a, miR$302 \mathrm{~b}$, miR-302c and miR-302d, were transiently overexpressed in the three types of pluripotent cells, ESCs, maGSCs and EGCs (Fig. 16).

\section{pre-miRNAs}

pos
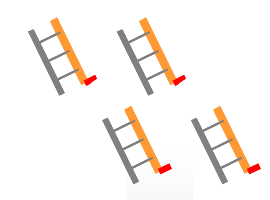

$+\mathbf{F L},+\mathbf{L I F}$

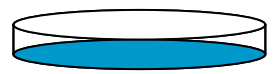

Undifferentiated cells

day 0

neg
-FL, -LIF

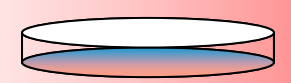

2

control pre-miRNA
-FL, -LIF

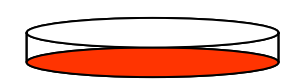

Differentiated cells day 10

Figure 16. Overexpression of miR-302 cluster in ESCs, maGSCs and EGCs. Overexpression of miR-302 cluster in these three cell lines was performed for 10 days in gelatine coated culture flasks without feeder layer (FL), LIF and RA. Cells were transfected either with precursor miRNA molecules of miR-302 cluster (positive, pos) or with a control precursor miRNA molecule without any biological function (negative, neg). 
Cells designated as positive cells (pos) were cultured on gelatine without feeder layer and LIF for 10 days and during this time they were transfected with all four pre-miRNAs of miR-302 cluster, miR-302a, miR-302b, miR-302c and miR-302d. These pre-miRNAs are in the form of chemically modified doublestranded RNA molecules designed to mimic endogenous mature miRNA molecules. The cells that were cultured under exactly the same conditions and transfected with a control pre-miRNA were the negative control (neg). This control pre-miRNA is a random sequence pre-miR molecule validated not to have any biological function and not to produce identifiable effects on known miRNA function. The transfection was repeated every 2 days (on days $0,2,4,6$ and 8); the cells were passaged on days $0,2,4,5$ and 10. Day 0 indicates undifferentiated cells that were subsequently used for differentiation and transfection.

Transfection conditions for overexpression of miRNAs in this cell system were optimized by using a Cy3 dye-labeled control pre-miRNA. Monitoring transfection efficiency by FACS analysis ensured that our transfectionretransfection scheme could maintain high transfection levels of the pre-miRNA (Fig. 17). 


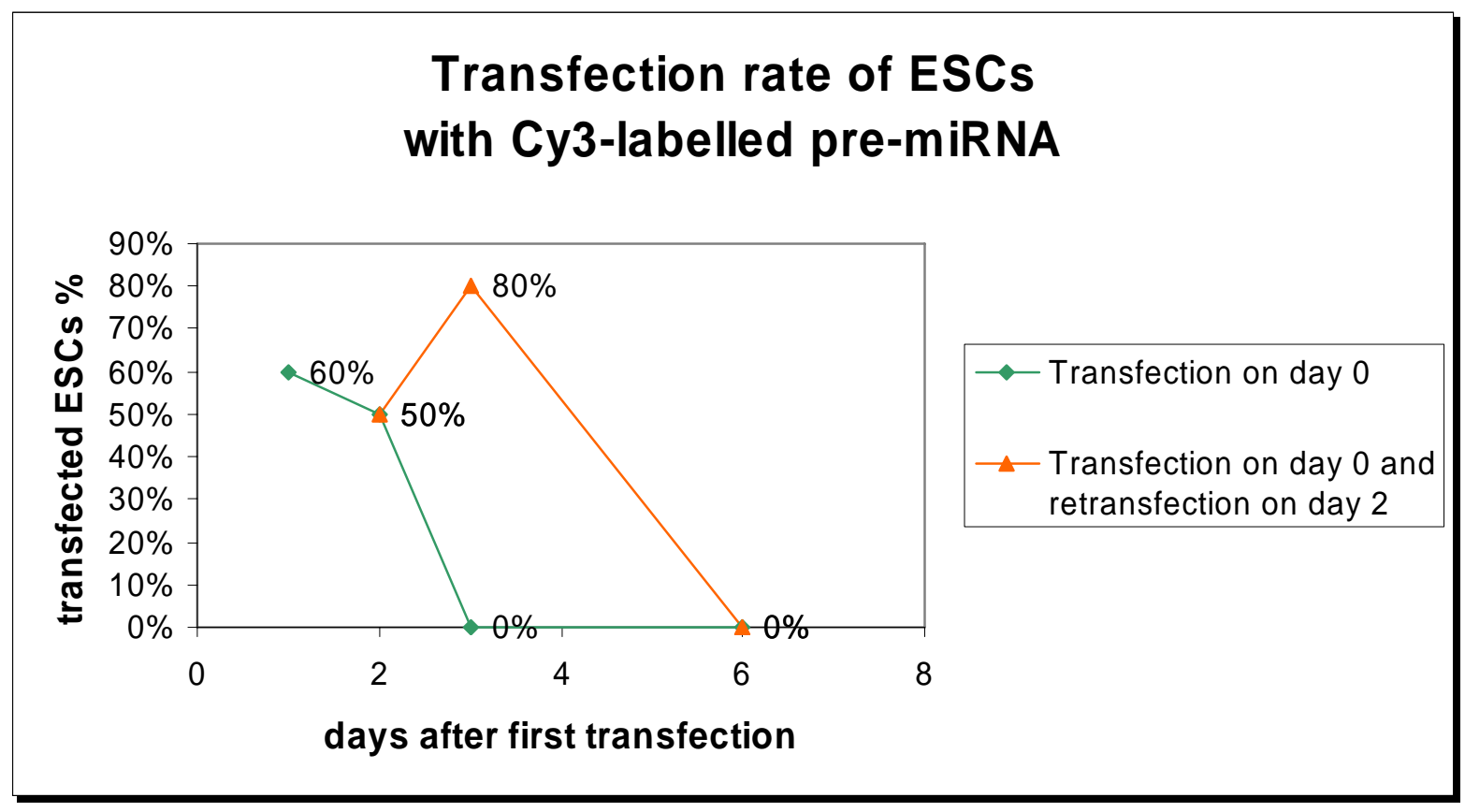

Figure 17. Optimization of transfection conditions in ESCs. ESCs were transfected with a $\mathrm{Cy} 3$ dye-labeled control pre-miRNA and the percentage of transfected cells was evaluated by FACS analysis. The first transfection took place on day 0; FACS analysis performed one, two and three days later after this transfection showed that transfection levels up to $50 \%$ of transfected ESCs were maintained until day 2 but not later (green line). When ESCs were retransfected with the same molecule 2 days after the first transfection, a transfection rate of $80 \%$ could be achieved on the following day (orange line).

In order to confirm the efficiency of the transfection, levels of miR-302a, miR$302 \mathrm{~b}$ and miR-302d were determined in ESCs on differentiating days 5 and 10 by qRT-PCR. The comparison to negative controls reveals a robust upregulation of all three miRNAs in positive samples (Fig. 18). 

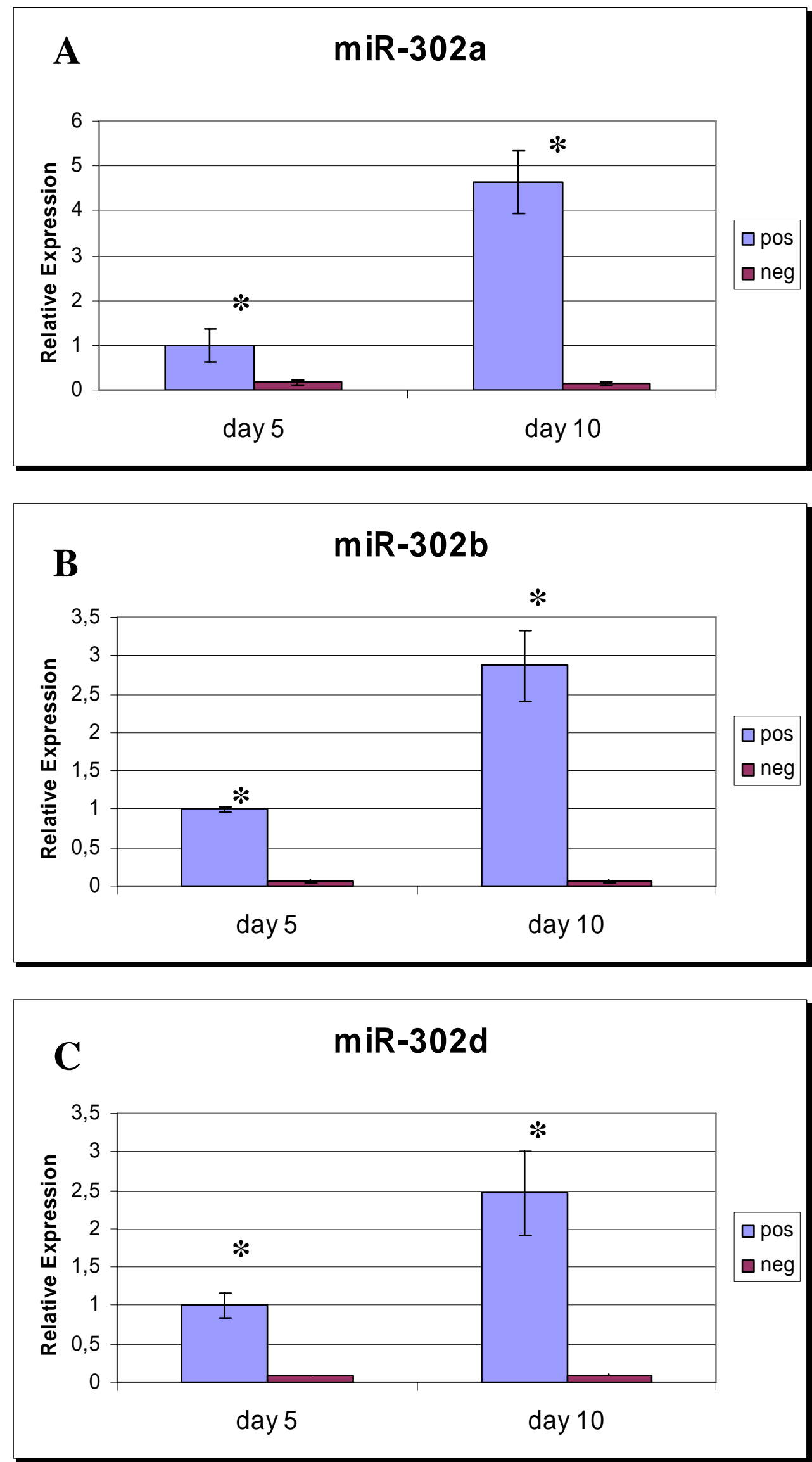
Figure 18. miR-302a (A), miR-302b (B) and miR-302d (C) in differentiating ESCs by overexpression of miR-302 cluster. miRNA expression levels of the three miRNAs were determined by real-time qRT-PCR in differentiating ESCs transfected with miR-302 cluster (pos) and a control pre-miRNA without any biological function (neg). The three miRNAs were tested on days 5 and 10 of differentiation. Expression levels were normalized to the level of the endogenous control (RNU6B). ESCs on day 5 of differentiation by overexpression of miR-302 cluster were used as calibrator, error bars indicate standard deviation. Asterisks indicate statistical significance for the difference between positive and the respective negative control cells.

\subsubsection{Overexpression of miR-302 cluster is not sufficient for maintenance of pluripotency}

In this experiment ESCs, maGSCs and EGCs were cultured under culture conditions that induce differentiation (on gelatine without feeder layer and LIF). Under such culture conditions it has been shown that ESCs and maGSCs undergo a significant reduction of expression levels of Oct4, a key gene widely used as pluripotency marker (Zovoilis et al. 2008).

It was of interest to examine whether miR-302 cluster can influence the expression of Oct4 under differentiation conditions for 10 days. Under these conditions, levels of Oct 4 were determined by qRT-PCR in cells where miR-302 cluster has been overexpressed (pos) and in the respective negative control cells (neg) (Fig. 19). 

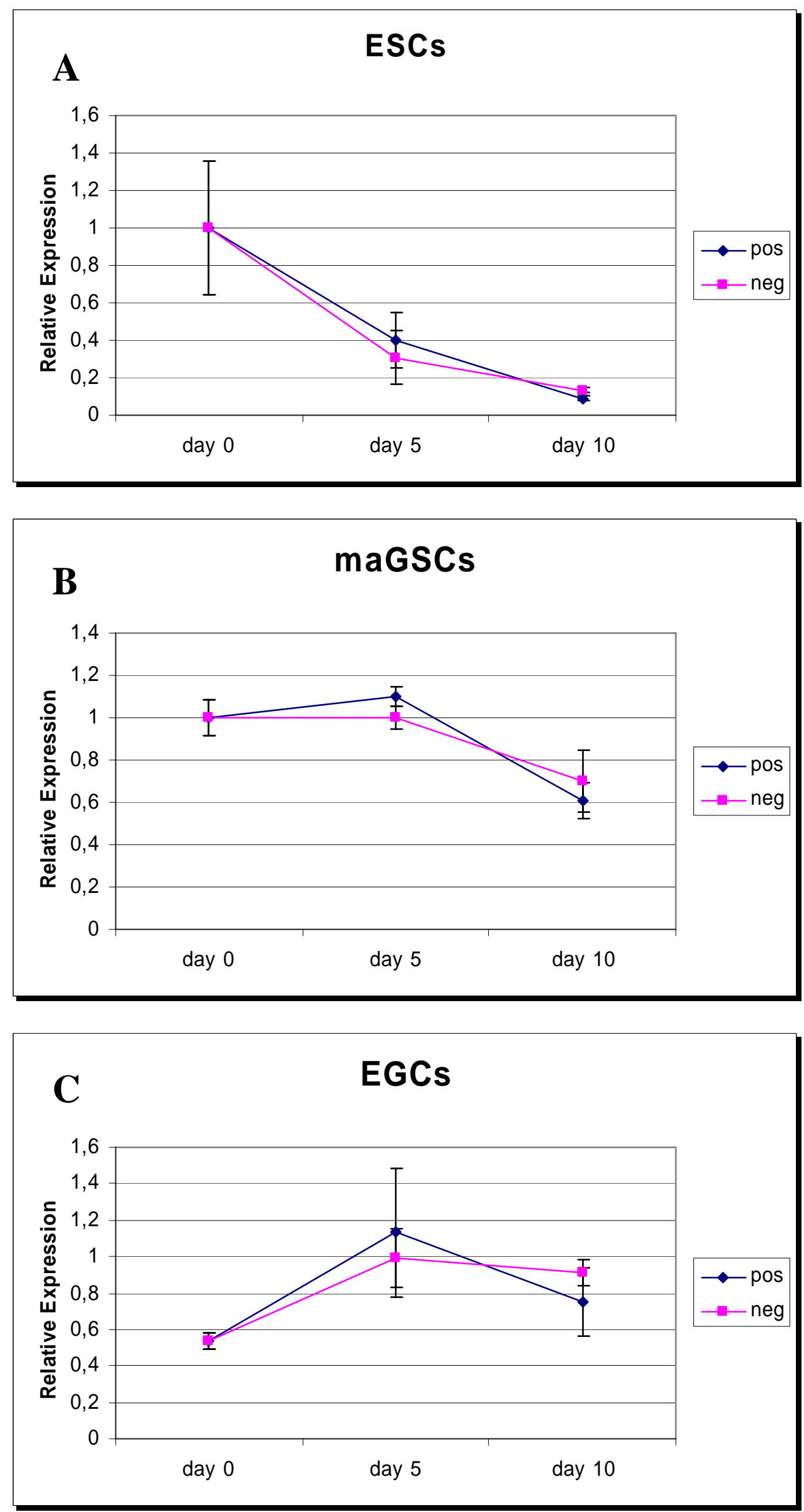
Figure 19. Oct4 in differentiating ESCs (A), maGSCs (B) and EGCs (C) by overexpression of miR-302 cluster. mRNA expression levels of Oct4 were determined by real-time qRT-PCR in undifferentiated cells (day 0) and in differentiating cells transfected with miR-302 cluster (pos) and a control premiRNA without any biological function (neg). The Oct4 mRNA was tested on days 5 and 10 of differentiation. Expression levels were normalized to the level of the endogenous control (succinate dehydrogenase complex, subunit A, flavoprotein, mSdha). Undifferentiated cells (day 0 ) were used as calibrator, error bars indicate standard deviation.

Figure 19 shows no significant difference between positive cells and negative controls for all three cell types on days 5 and 10 .

It should be emphasized that in ESCs levels of Oct4 during differentiation decrease dramatically (negative control). In this case it was obvious that these miRNas were not able to maintain pluripotent state. Contrary to ESCs, maGSCs and EGCs retain high levels of Oct 4 even after 10 days of growth under gelatine culture condition. In this case miR-302 cluster overexpression did not have any impact on Oct4 expression profile. 


\subsubsection{Influence of miR-302 cluster on in vitro differentiation of pluripotent cells}

The undifferentiated status of ESCs and, likewise, of other pluripotent cells, can be maintained in vitro in the presence of feeder layer and LIF. By removing feeder layer and LIF, ESCs tend to achieve multi-lineage differentiation.

In order to examine the influence of miR-302 cluster on the differentiation of pluripotent cells, such as ESCs, maGSCs and EGCs, expression of markers that characterize mesoderm, ectoderm and germ cell lineage were examined. The miR-302 cluster overexpression was conducted with the use of serum-containing medium, in the absence of feeder layer and LIF in a cell system described in a previous work (Zovoilis et al. 2008). In this cell system, which is also used in the present study, after 10 days of differentiation ESCs did not show any significant increase in levels of endoderm markers, so these markers were not tested here.

Levels of the following genes were tested with qRT-PCR on days 5 and 10 of culture:

- Brachyury (also known as T) (Fig. 20), eomesodermin and fibroblast growth factor 8 (Fgf-8) (both tested only in ESCs) (Fig. 21), three genes that are required for mesoderm formation and gastrulation (Keller 2005; Alisch et al. 2007),

- Nestin (Fig. 22), an early neuroectodermal marker (Lendahl et al. 1990), and 
- Dppa3 (also known as Pgc7 or stella) (Fig. 23), a gene expressed in primordial germ cells, with a central role in germ cell specification (Tres et al. 2004).

\subsubsection{Markers of mesoderm: Brachyury, Eomesodermin and Fgf-8}
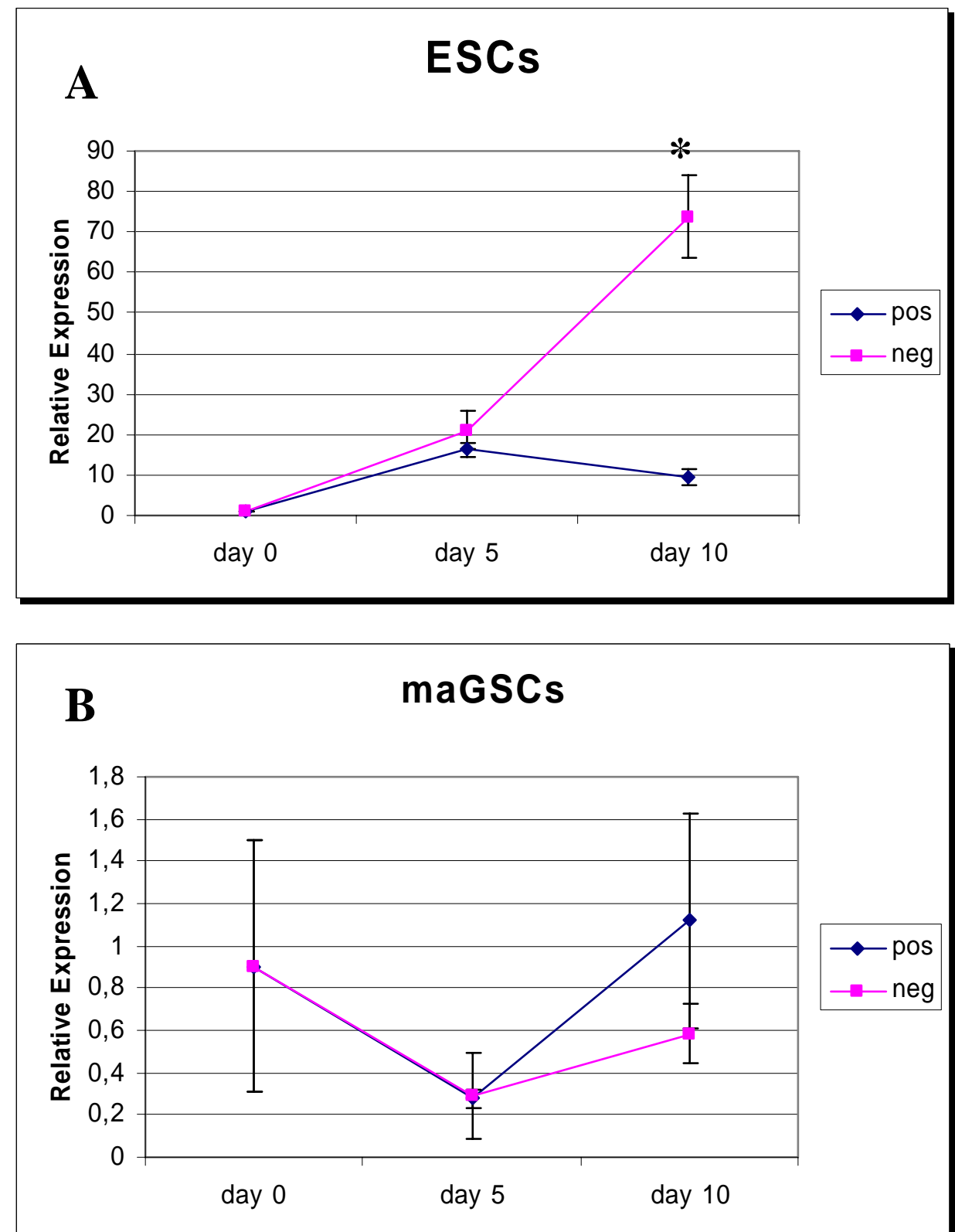


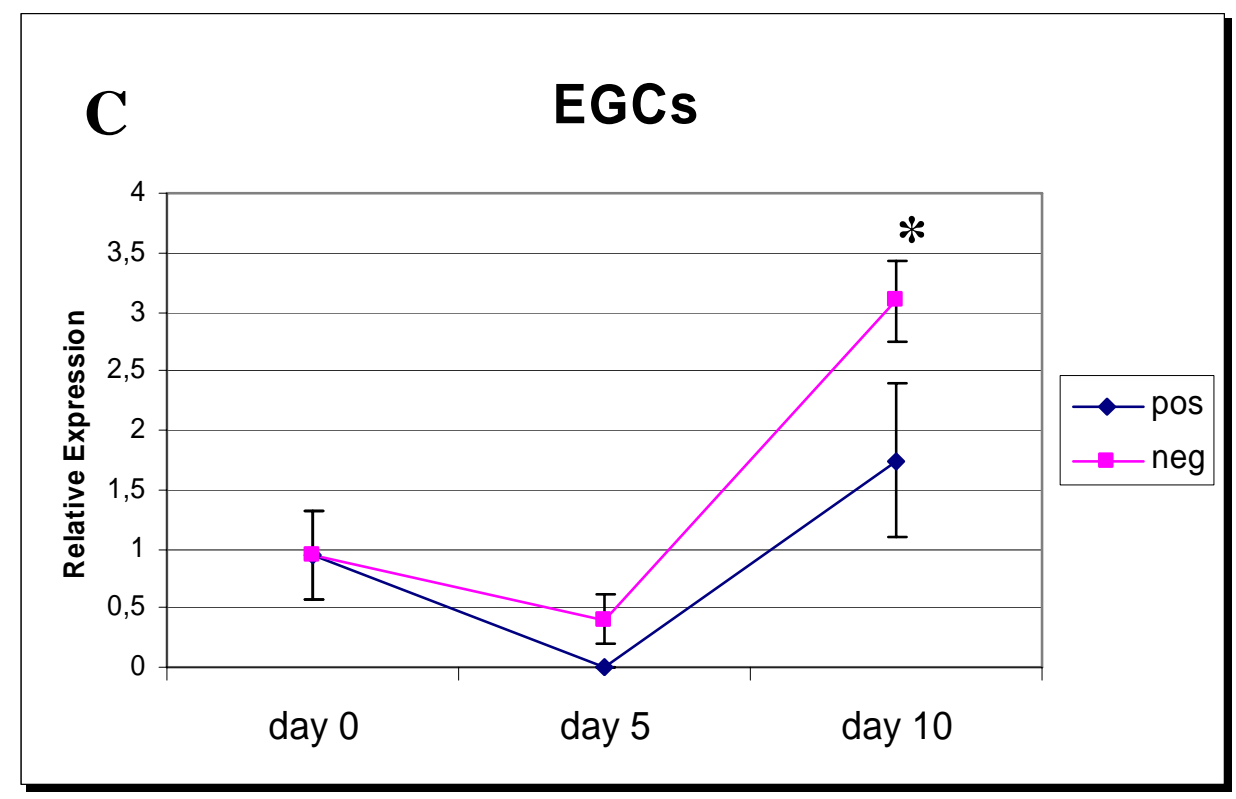

Figure 20. Brachyury in differentiating ESCs (A), maGSCs (B) and EGCs (C) by overexpression of miR-302 cluster. mRNA expression levels of Brachyury were determined by real-time qRT-PCR in undifferentiated cells (day 0 ) and in differentiating cells transfected with miR-302 cluster (pos) and with a control pre-miRNA without biological function (neg). The Brachyury mRNA was tested on days 5 and 10 of differentiation. Expression levels were normalized to the level of the endogenous control (succinate dehydrogenase complex, subunit A, flavoprotein, mSdha). Undifferentiated cells (day 0) were used as calibrator, error bars indicate standard deviation. Asterisks indicate statistical significance for the difference between positive and the respective negative control cells.

Figure 20 indicates that overexpression of miR-302 cluster has a significant effect on the Brachyury expression in ESCs and EGCs. Although until day 5 no significant difference exists between positive samples and negative controls, on day 10 ESCs display a strong inhibition of Brachyury expression. EGCs follow a similar pattern with the ESCs but the inhibition of Brachyury occurs to a 
smaller extent. These data suggest that Brachyury, a gene with pivotal role in mesoderm formation and organization, is downregulated by miR-302 cluster. In maGSCs Brachyury is poorly expressed neither in undifferentiated cells nor on days 5 and 10, resulting in unreliable qRT-PCR data with considerable standard deviation.

The hypothesis that miR-302 cluster inhibits the mesoderm formation is further supported by data concerning two further genes necessary for mesoderm formation and gastrulation, Eomesodermin and Fgf-8 (Alisch et al. 2007). These genes were examined in ESCs by qRT-PCR (Fig. 21). Both Eomesodermin and Fgf- 8 display the same profile as Brachyury, with a significant inhibition of their expression mainly on day 10 .

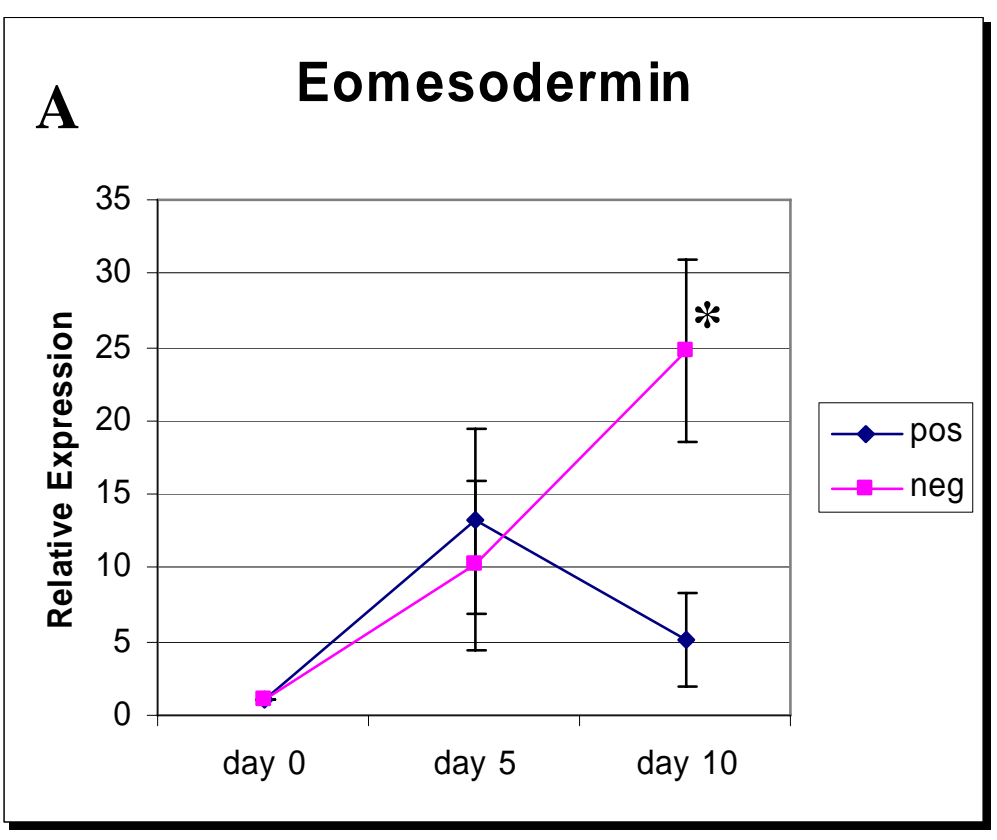




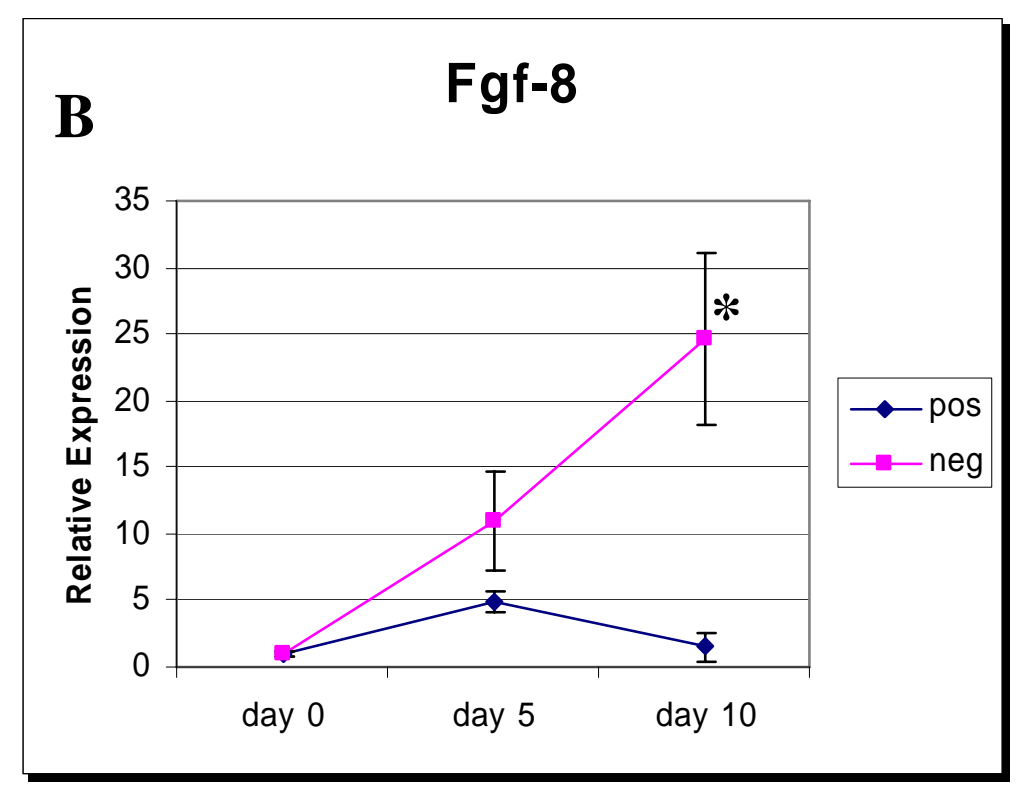

Figure 21. Eomesodermin and Fibroblast Growth Factor 8 (Fgf-8) in differentiating ESCs by overexpression of miR-302 cluster. mRNA expression levels of Eomesodermin (A) and Fgf-8 (B) were determined by realtime qRT-PCR in undifferentiated ESCs (day 0) and in differentiating ESCs transfected with miR-302 cluster (pos) and with a control pre-miRNA without biological function (neg). Both mRNAs were tested on days 5 and 10 of differentiation. Expression levels were normalized to the level of the endogenous control (succinate dehydrogenase complex, subunit A, flavoprotein, mSdha). Undifferentiated ESCs (day 0) were used as calibrator, error bars indicate standard deviation. Asterisks indicate statistical significance for the difference between positive and the respective negative control cells.

In maGSCs and EGCs under the differentiation conditions used in this study Fgf- 8 and Eomesodermin were not detectable by RT-PCR and were not tested by qRT-PCR. 


\subsubsection{Nestin: marker of ectoderm}

Nestin is highly expressed in neural progenitor cells (Lendahl et al. 1990) and was used in this study as a neuroectodermal marker. qRT-PCR data revealed no difference between positively transfected cells and negative controls, suggesting that overexpression of miR-302 cluster in ESCs does not alter their differentiation towards the neuroectodermal lineage (Fig. 22).

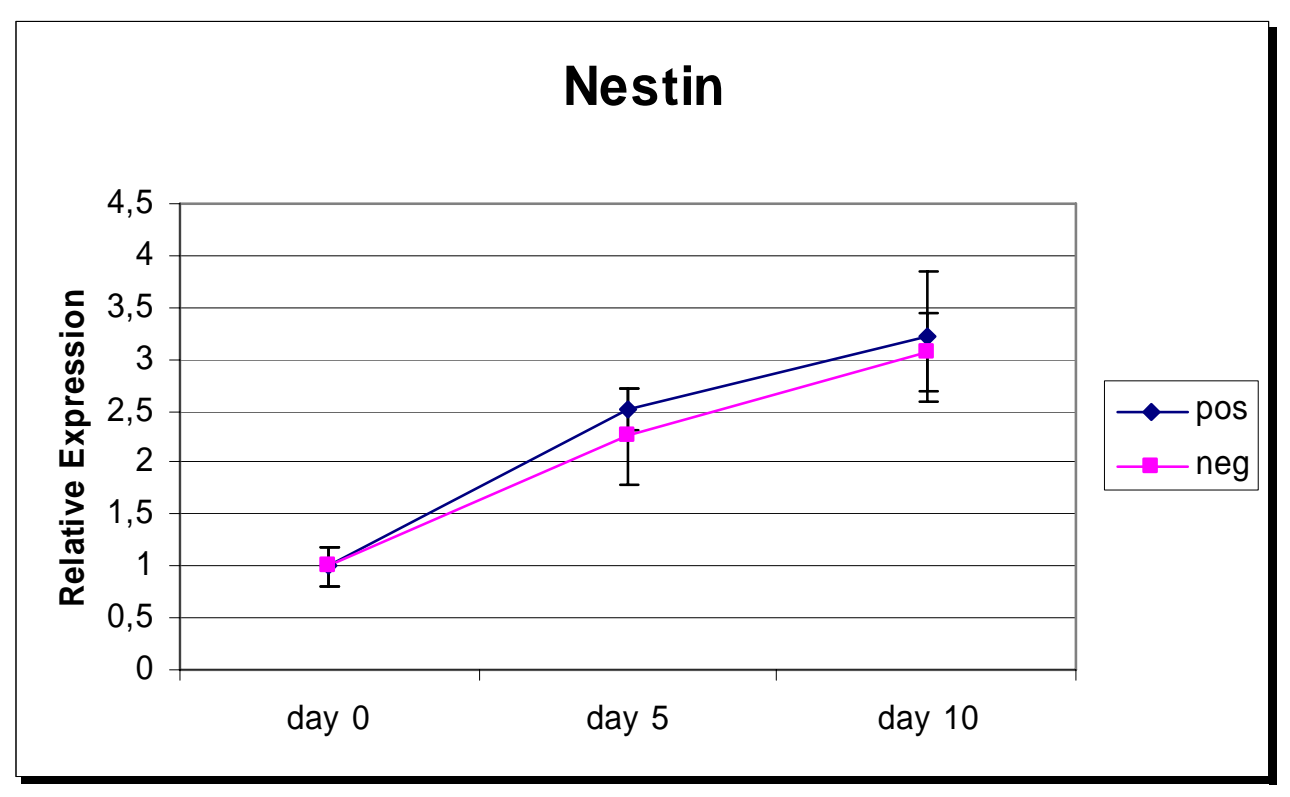

Figure 22. Nestin in differentiating ESCs by overexpression of miR-302 cluster. mRNA expression levels of Nestin were determined by real-time qRTPCR in undifferentiated ESCs (day 0) and in differentiating ESCs transfected with miR-302 cluster (pos) and with a control pre-miRNA without biological function (neg). The Nestin mRNA was tested on days 5 and 10 of differentiation. Expression levels were normalized to the level of the endogenous control (succinate dehydrogenase complex, subunit A, flavoprotein, mSdha). Undifferentiated ESCs (day 0) were used as calibrator, error bars indicate standard deviation. 


\subsubsection{Dppa3: marker of germ cell lineage}

Primordial germ cells are the progenitors of gametes and their specification begins in the epiblast during gastrulation in mouse. After migration, germ cells arrive at the developing gonads, where they ultimately produce spermatozoa and oocytes, respectively (Tres et al. 2004). Dppa3, a gene detected mainly in primordial germ cells, was used in this study to examine which influence the overexpression of miR-302 cluster has on the differentiation of pluripotent cells towards the germ line.
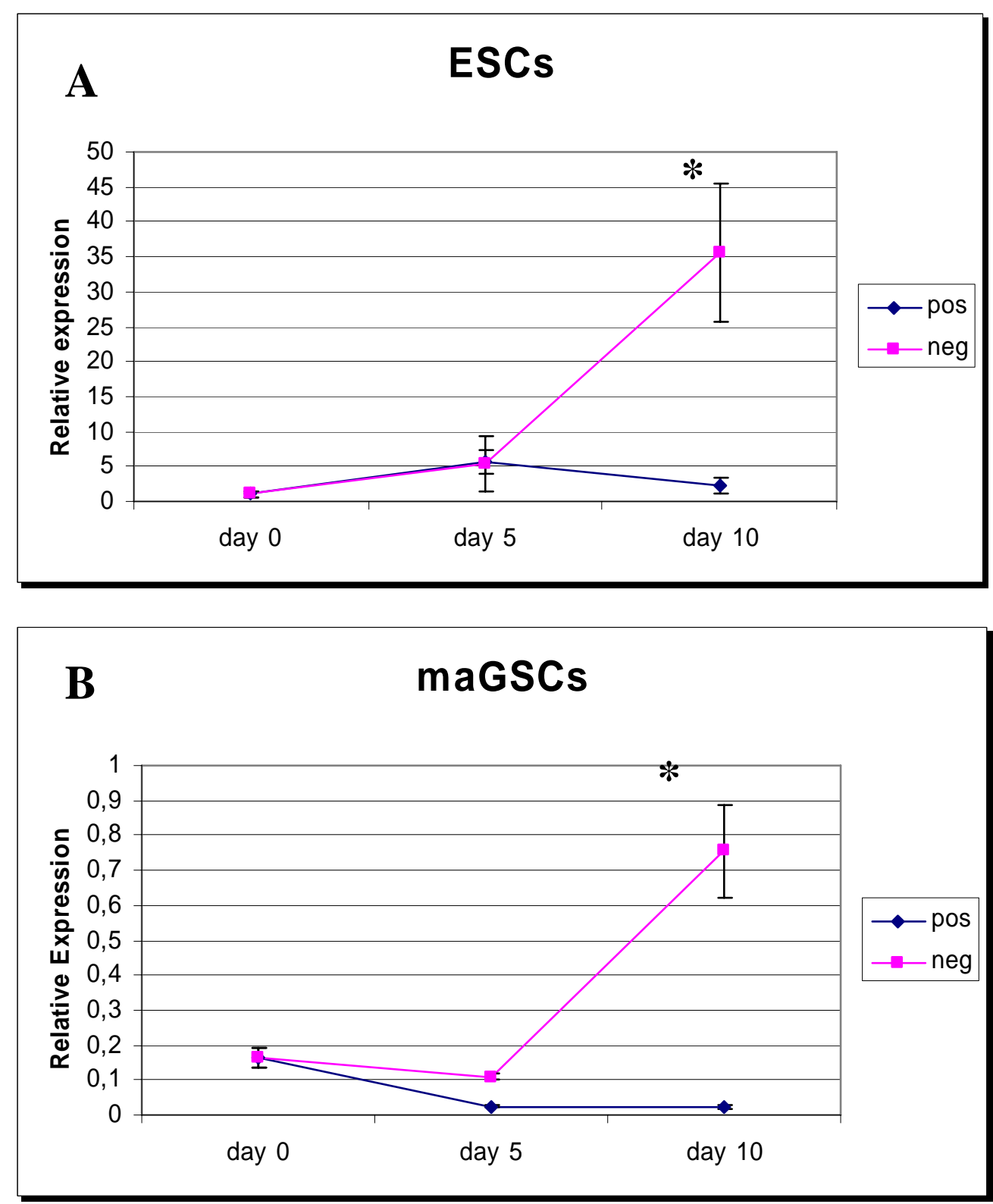


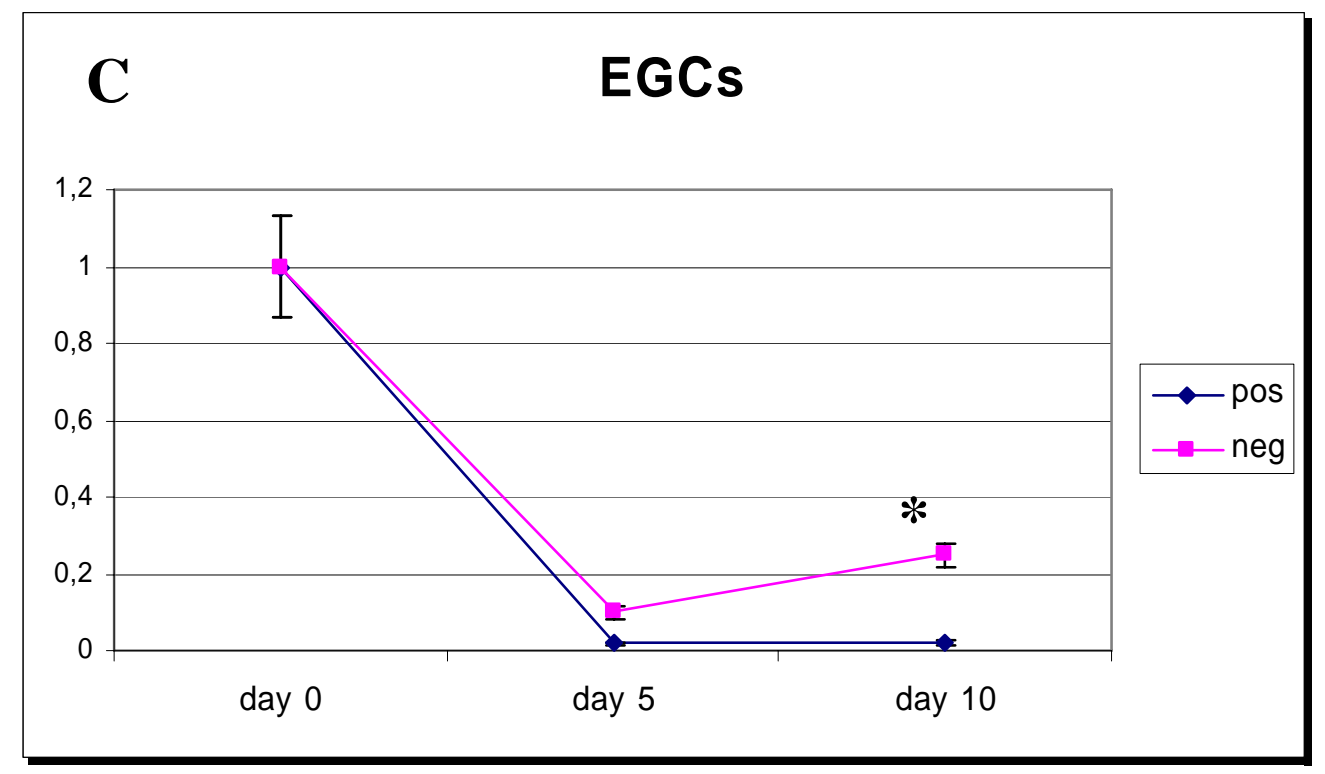

Figure 23. Dppa3 in differentiating ESCs (A), maGSCs (B) and EGCs (C) by overexpression of miR-302 cluster. mRNA expression levels of Dppa3 were determined by real-time qRT-PCR in undifferentiated cells (day 0 ) and in differentiating cells transfected with miR-302 cluster (pos) and with a control pre-miRNA without biological function (neg). The Dppa3 mRNA was tested on days 5 and 10 of differentiation.. Expression levels were normalized to the level of the endogenous control (succinate dehydrogenase complex, subunit A, flavoprotein, mSdha). Undifferentiated cells (day 0 ) were used as calibrator, error bars indicate standard deviation. Asterisks indicate statistical significance for the difference between positive and the respective negative control cells.

ESCs and maGSCs that are submitted to miR-302 cluster overexpression show an exceptional downregulation of Dppa3 gene on day 10, suggesting that miR302 cluster inhibits Dppa3 expression and further differentiation of ESCs and maGSCs towards the germ line. From day 0 until day 5 no significant difference between positive and negative controls is observed (Fig. 23A and B).

EGCs present elevated initial mRNA levels of Dppa3, which drop dramatically until day 5 and remain low until day 10. Positively transfected cells and negative control show also significant differences (Fig. 23C). 


\subsubsection{Evaluation of possible targets of miR-302 cluster}

\subsubsection{Prediction of possible targets of miR-302 cluster by TargetScan}

As shown in figures 20 and 22, overexpression of miR-302 cluster leads to downregulation of Brachyury in ESCs and EGCs and of Dppa3 in ESCs, maGSCs and EGCs. However, according to TargetScan/Release 4.2 (http://www.targetscan.org/vert 42/), a bioinformatics web tool that predicts biological targets of miRNAs, miR-302 cluster is not predicted to target Brachyury or Dppa3 (Fig. 24).

\section{OPargetScan

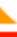

\section{Mouse T 3' UTR}

A

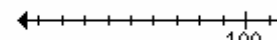

Gene

Mouse T NM_003181 $3^{\prime}$ UTR length:656

Conserved sites for niRNA fanilies conserved in Hunan, Mouse, Rat, Dog, and Chicken

\section{Mouse DPPA3 3' UTR}

B

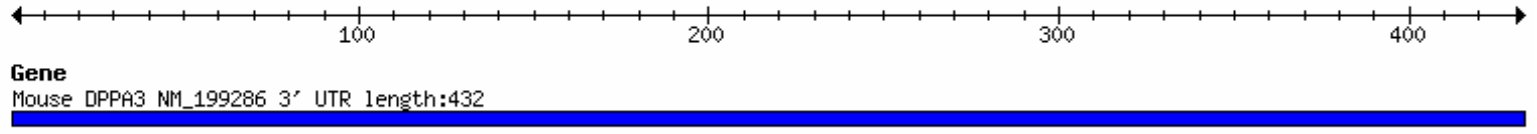

Conserved sites for niRM fanilies conserved in Hunan, Mouse, Rat, Dog, and Chicken 
Figure 24. Predicted miRNAs that target the mRNAs of Brachyury (A) and Dppa3 (B) according to TargetScan/Release 4.2. TargetScan predicts biological targets of miRNAs by searching for the presence of conserved sites that match the seed region of each miRNA. A: Two predicted miRNAs (miR-219 and miR-9) target Brachyury (also known as T) and are designated as conserved among tested species (human, mouse, rat, dog, chicken). B: TargetScan search delivered no results for sites conserved among tested species in the case of Dppa3. Members of miR-302 cluster are not predicted to target Brachyury or Dppa3 (Source: http://www.targetscan.org/vert 42/).

Additionally, TargetScan search for predicted targets of miR-302 cluster delivered 445 conserved targets that did not include Brachyury or Dppa3 (see http://www.targetscan.org/vert 42/). Since Brachyury and Dppa3 are markers for the mesodermal and the germ cell lineage respectively (Keller 2005; Tres et al. 2004), this study has further focused on signalling pathways that control the establishment of both lineages such as Bmp4, Nodal and Wnt (Yabuta et al. 2006; Tam and Loebel 2007). During formation of both mesoderm and germ cell lineage activation of all three pathways is required (Hayashi et al. 2007).

Bmp4 and Nodal are members of the TGFß protein superfamily and require the mediation of intracellular Smad proteins in order to regulate mesoderm and germ cell lineage formation (Fig. 25) (Chen et al. 2004; Sudheer and Adjaye 2007). 


\section{A}

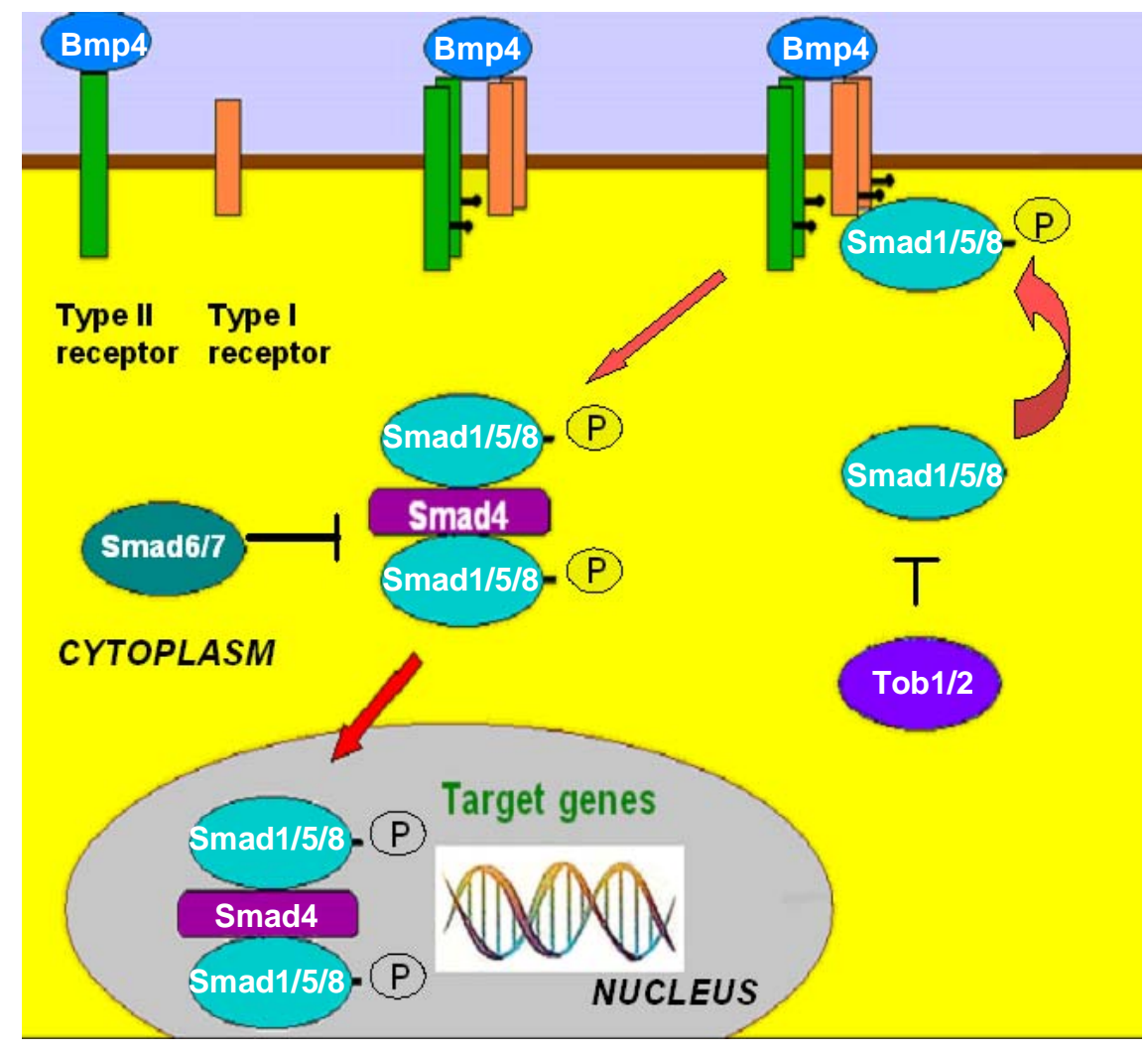

B

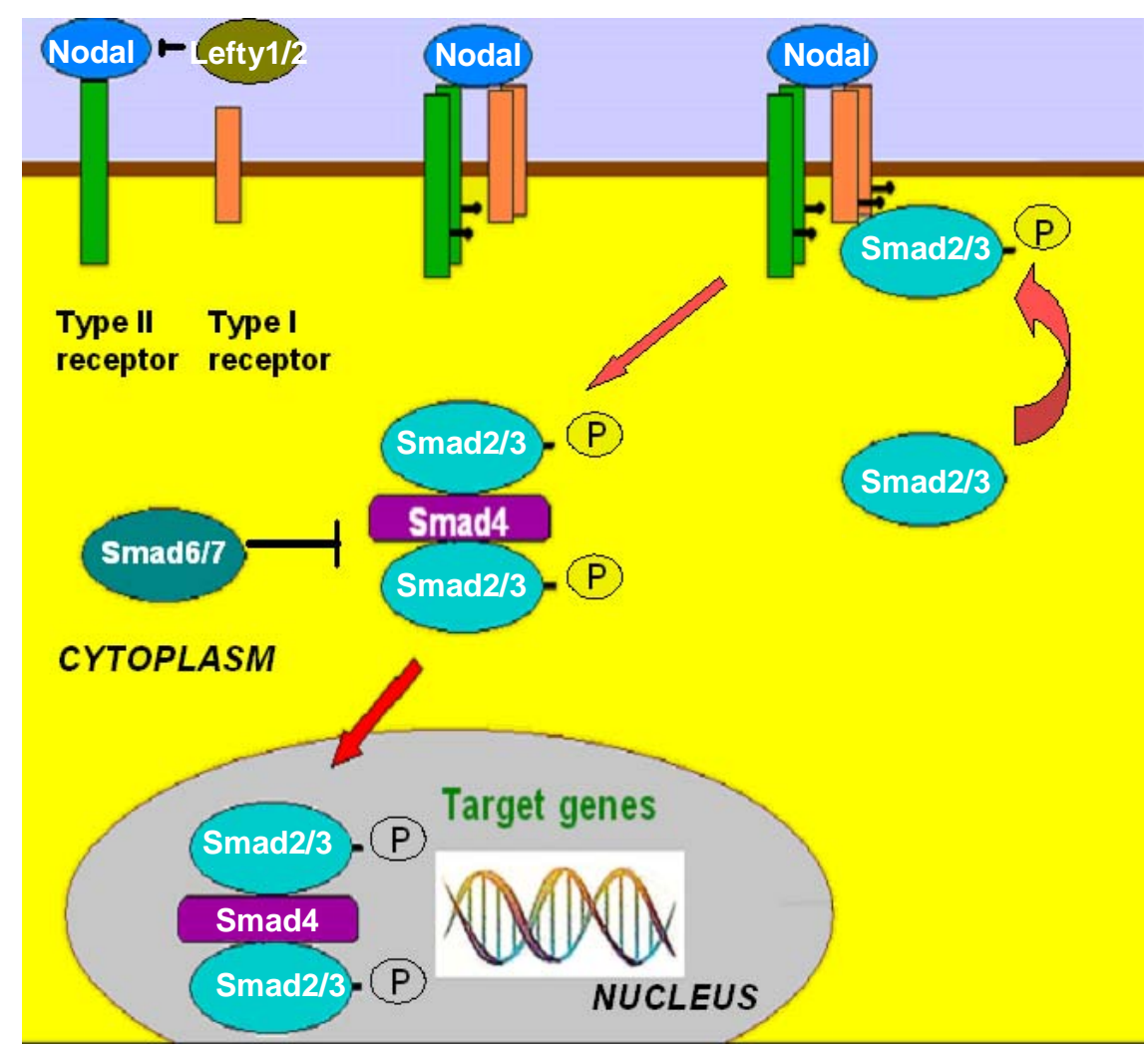


Figure 25. Bmp4 and Nodal signalling. Bmp4 (A) and Nodal (B) signals are mediated by type I and II Bmp4 and Nodal receptors, respectively, and their downstream phosphorylated molecules (Smad1, Smad5 and Smad8 for Bmp4, Smad2 and Smad3 for Nodal) form a complex with the common-mediator Smad4. The protein complex enters the nucleus and interacts with transcription factors (target genes), regulating mesoderm and germ cell lineage formation. The Bmp4 and Nodal pathways are targeted by various inhibitors as the common Smad6 and Smad7, Tob1 and Tob2 for Bmp4 pathway and Lefty1 and Lefty2 for Nodal pathway (adapted from Chen et al. 2007).

Wnt/ß-catenin pathway also targets expression of genes necessary for the development of mesoderm and germ cell lineage. One key antagonist of this pathway is Dkk1, a protein that inhibits interaction between Wnt and its membrane receptor (Tam and Loebel 2007).

According to TargetScan, five genes of all tested mediators or inhibitors of Bmp4, Nodal and Wnt pathways are retrieved as possible targets of miR-302 cluster (Table 3 ) and are the following:

- Smad2, a Nodal activator,

- Dkk1, a Wnt inhibitor,

- Lefty1 and Lefty2, two Nodal inhibitors, and

- Tob2, a Bmp4 inhibitor. 


\begin{tabular}{|c|c|c|}
\hline Genes & Function & Predicted target of miR-302 cluster \\
\hline Smad1 & Bmp4 activator & No \\
\hline Smad5 & Bmp4 activator & No \\
\hline Smad8 & Bmp4 activator & Yes \\
\hline Smad2 & Nodal activator & No \\
\hline Smad3 & Nodal activator & No \\
\hline Smad4 & Bmp4/Nodal activator & No \\
\hline Smad6 & Bmp4/Nodal inhibitor & No \\
\hline Smad7 & Bmp4/Nodal inhibitor & No \\
\hline Tob1 & Bmp4 inhibitor & Yes \\
\hline Tob2 & Bmp4 inhibitor & Yes \\
\hline Lefty1 & Nodal inhibitor & Yes \\
\hline Lefty2 & Nodal inhibitor & Yes \\
\hline Dkk1 & Wnt inhibitor & \\
\hline
\end{tabular}

Table 3. Predicted targets of miR-302 cluster among activators+/inhibitors of Bmp4, Nodal and Wnt pathways according to TargetScan/Release 4.2.

After screening several activators and inhibitors of the three pathways, only five of them (red colour) contained, according to TargetScan (http://www.targetscan.org/vert 42/), target sites for miR-302 cluster in their 3'UTR conserved in all tested vertebrates (human, mouse, rat, dog, chicken). 


\subsubsection{Use of Luciferase assay for confirmation of predicted targets of miR-302 cluster}

In order to confirm some of the computationally predicted targets of miR-302 cluster, two of them, Smad2 and Dkk1, were selected and tested by a Luciferase assay.

This assay was based on measuring the firefly luciferase activity produced by a plasmid that included the open reading frame (ORF) and the 3' UTR sequences of Smad2 or Dkk1 downstream of the Luciferase translation sequence. Expression of the Luciferase gene would lead to emission of light signal (Scheme 2A). In the presence of a miRNA that could target the 3' UTR of Smad2 or Dkk1 (in this case members of miR-302 cluster), the light signal emission would be reduced (Schema 2B).

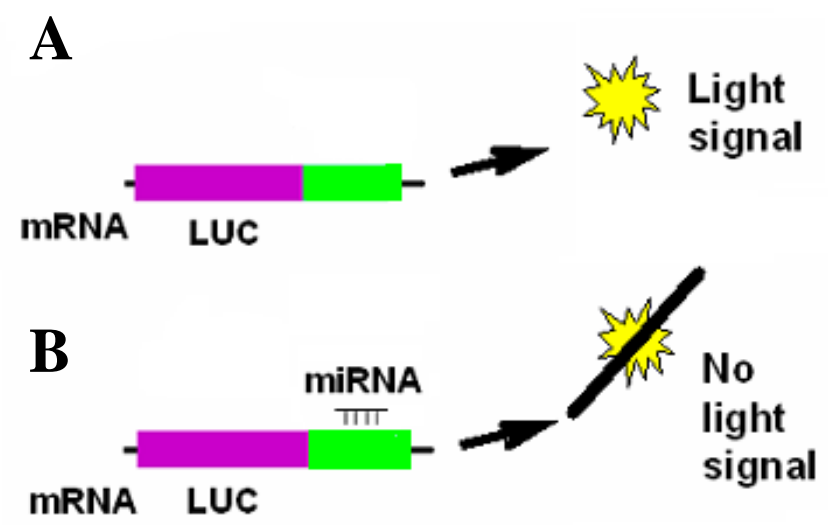

Scheme 2. Firefly Luciferase expression. (A) The Luciferase translation sequence is illustrated with pink colour and its 3' UTR with green. Expression of the enzyme luciferase leads to light signal after introduction of the substrate luciferin in the following reaction (two steps): Beetle Luciferin + ATP $\rightarrow$ Luciferyl adenylate $+\mathrm{PP}_{\mathrm{i}}$; Luciferyl adenylate $+\mathrm{O}_{2} \rightarrow$ Oxyluciferin $+\mathrm{AMP}+$ 
Light. (B) In the presence of a miRNA that targets downstream the Luciferase mRNA containing the 3' UTR sequence of Smad2 or Dkk1 in its 3' UTR (green colour), mRNA translation is repressed and light signal emission decreases.

\subsubsection{Generation of miRNA Luciferase Vectors}

In cooperation with Mr. L.Smorag, Luciferase Vectors for the genes Smad2 and Dkk1 were constructed as following: first, the 3' UTR sequences of Smad2 and Dkk1 were amplified with gene-specific primers containing restriction sites for the enzymes SacI (SstI) and HindIII in their 5' ends. Subsequently, the amplification products were cloned into pGEMT Easy Vector and this vector was then digested with the restriction enzymes SacI (SstI) and HindIII. Last, the digestion product was cloned in the miRNA Luciferase Vector, which was previously linearized by SacI (SstI) and HindIII.

The miRNA Expression Reporter Vector System that was used consists of the miRNA Luciferase Vector and an associated B-gal control plasmid. The miRNA Luciferase Vector contains the ORF of Luciferase under the control of a cytomegalovirus (CMV) promoter. Downstream of the Luciferase translation sequence the vector includes a multiple cloning site (MCS) with restriction sites for, among others, SacI (SstI) and HindIII. Here the 3' UTR sequences for Smad2 (CMV-LUC-Smad2 3' UTR) and Dkk1 (CMV-LUC-Dkk1 3' UTR) were cloned (Scheme 3). 


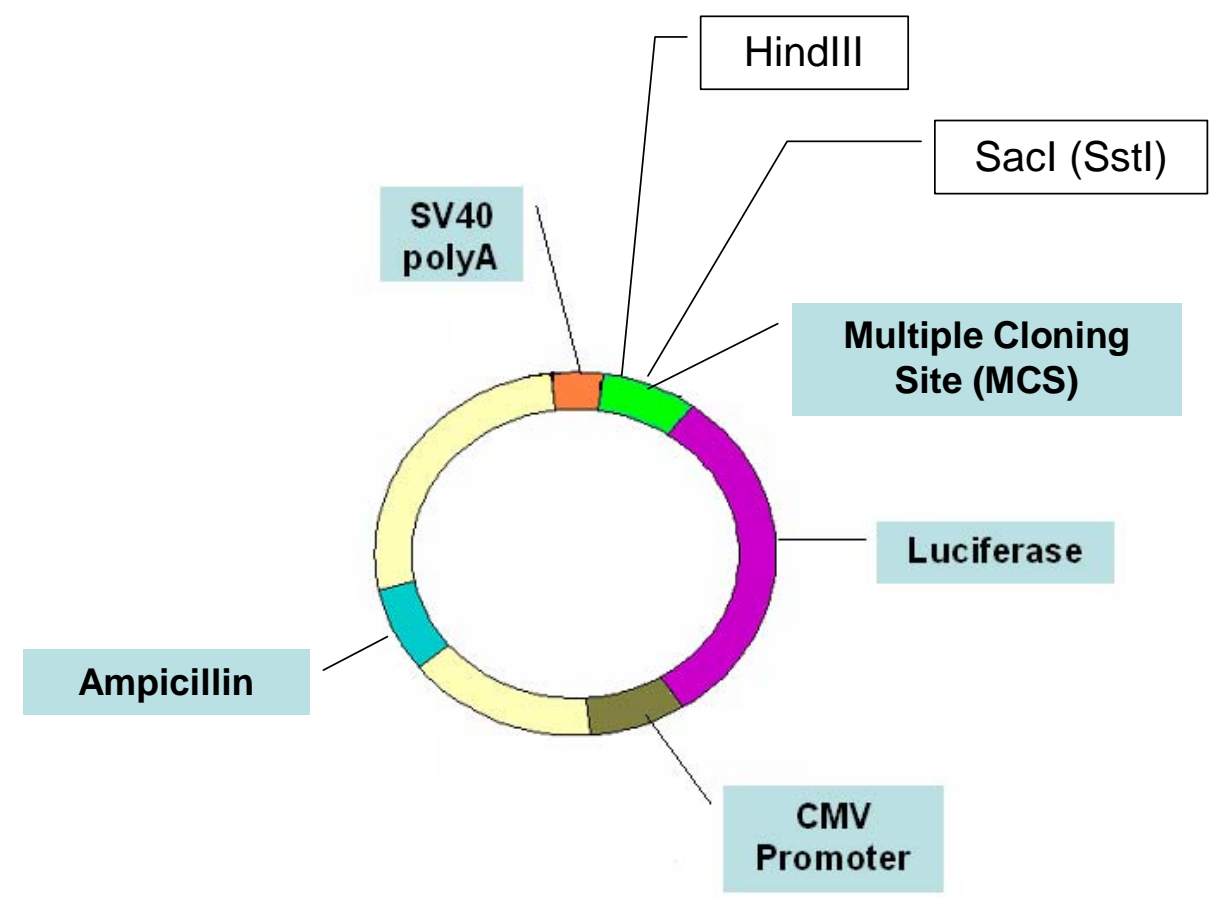

Scheme 3. miRNA Luciferase Vector. The miRNA Luciferase Vector expresses firefly Luciferase (pink colour) under the control of CMV promoter (brown colour). The plasmid was linearized by digestion in the multiple cloning site (MCS, green colour) with SacI (SstI) and HindIII enzymes. Then two vectors were generated, one with the cloning of the 3' UTR sequence of Smad2 (CMV-LUC-Smad2 3' UTR) and one with the cloning of the 3' UTR sequence of Dkk1 (CMV-LUC-Dkk1 3' UTR) downstream of the Luciferase ORF.

\subsubsection{Establishment of the Luciferase assay}

In order to check the efficiency of the two generated miRNA Luciferase Vectors for Smad2 (CMV-LUC-Smad2 3' UTR) and Dkk1 (CMV-LUC-Dkk1 3' UTR), each vector was transfected into NIH/3T3 cells and, according to manufacturer's instructions, $24 \mathrm{hrs}$ after the transfection luciferase activity of the transfected cells was measured and compared to luciferase activity of untransfected NIH/3T3 cells. Cells transfected with each of the miRNA Luciferase Vectors demonstrated a significantly higher luciferase activity in comparison to untransfected cells (Fig. 26). 

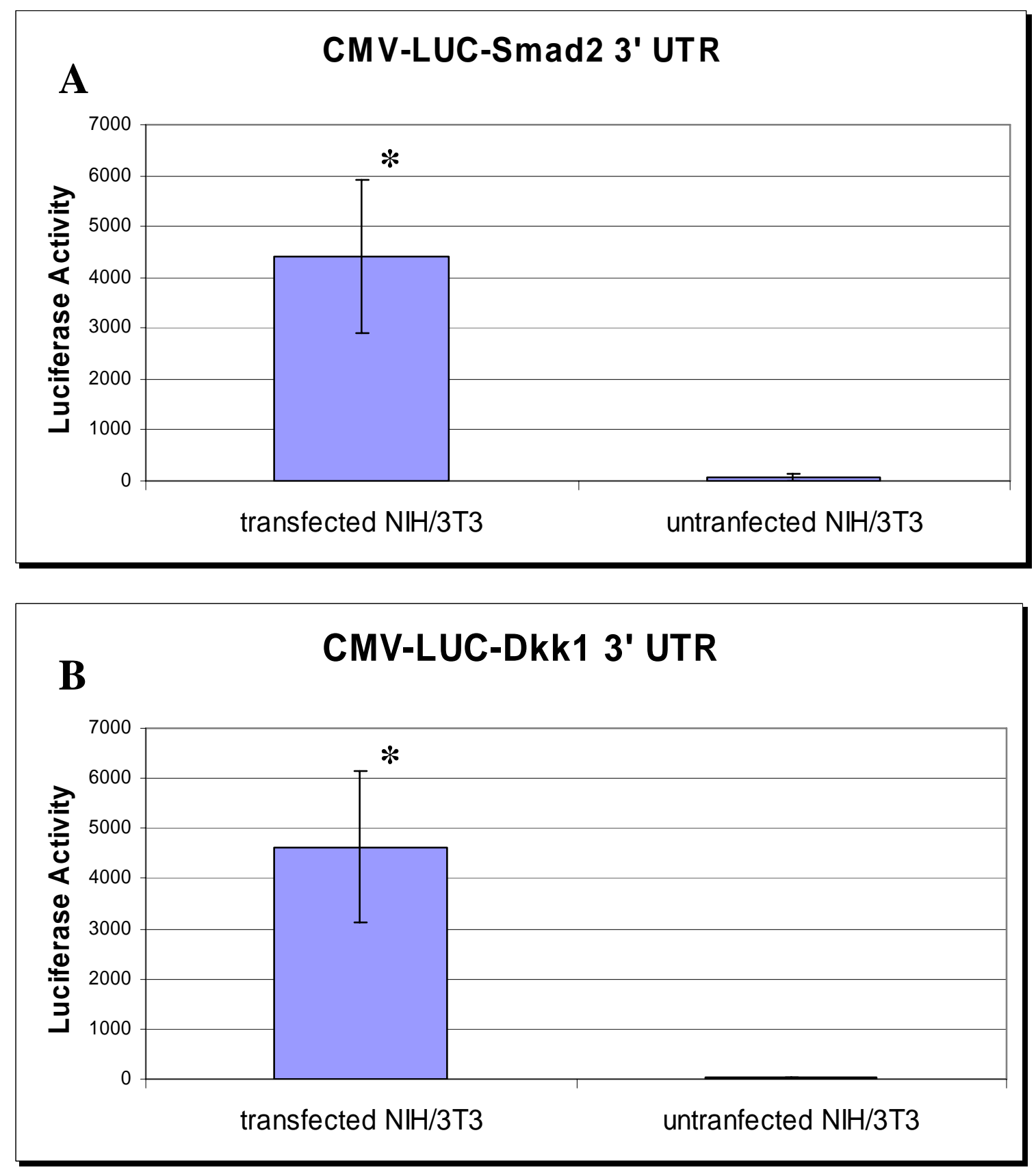

Figure 26. Validation of generated miRNA Luciferase Vectors for Smad2 (CMV-LUC-Smad2 3' UTR) (A) and Dkk1 (CMV-LUC-Dkk1 3' UTR) (B). NIH/3T3 cells that were transfected with each of the miRNA Luciferase Vectors demonstrated a significantly higher luciferase activity in comparison to untransfected NIH/3T3 cells. Error bars indicate standard deviation. Asterisks indicate statistical significance for the difference between transfected and the respective untransfected NIH/3T3 cells. 


\subsubsection{Experimental layout and results of the Luciferase assay}

$\mathrm{NIH} / 3 \mathrm{~T} 3$ cells were contransfected with:

- The miRNA Luciferase Vector containing the 3' UTR of Smad2 (CMVLUC-Smad2 3' UTR) or Dkk1 (CMV-LUC-Dkk1 3' UTR),

- the $\beta$-gal control plasmid for normalization and

$>$ four pre-miRNAs, each for one of the four members of miR-302 cluster, miR-302a, miR-302b, miR-302c and miR-302d, in the form of chemically modified double-stranded RNA molecules designed to mimic endogenous mature miRNA molecules. The cells that were transfected with these pre-miRNAs were the positive sample (pos). The same pre-miR molecules were used in the overexpression of miR-302 cluster in pluripotent cells (see 4.3).

or a control pre-miRNA, which is a random sequence pre-miR molecule validated not to have any biological function and not to produce identifiable effects on known miRNA function. The cells that were transfected with the control pre-miRNA were the negative control (neg). The same negative control was used in the overexpression of miR-302 cluster in pluripotent cells (see 4.3).

Figures 27 and 28 show that Smad2 and Dkk1 genes are not targeted by miR302 cluster. 


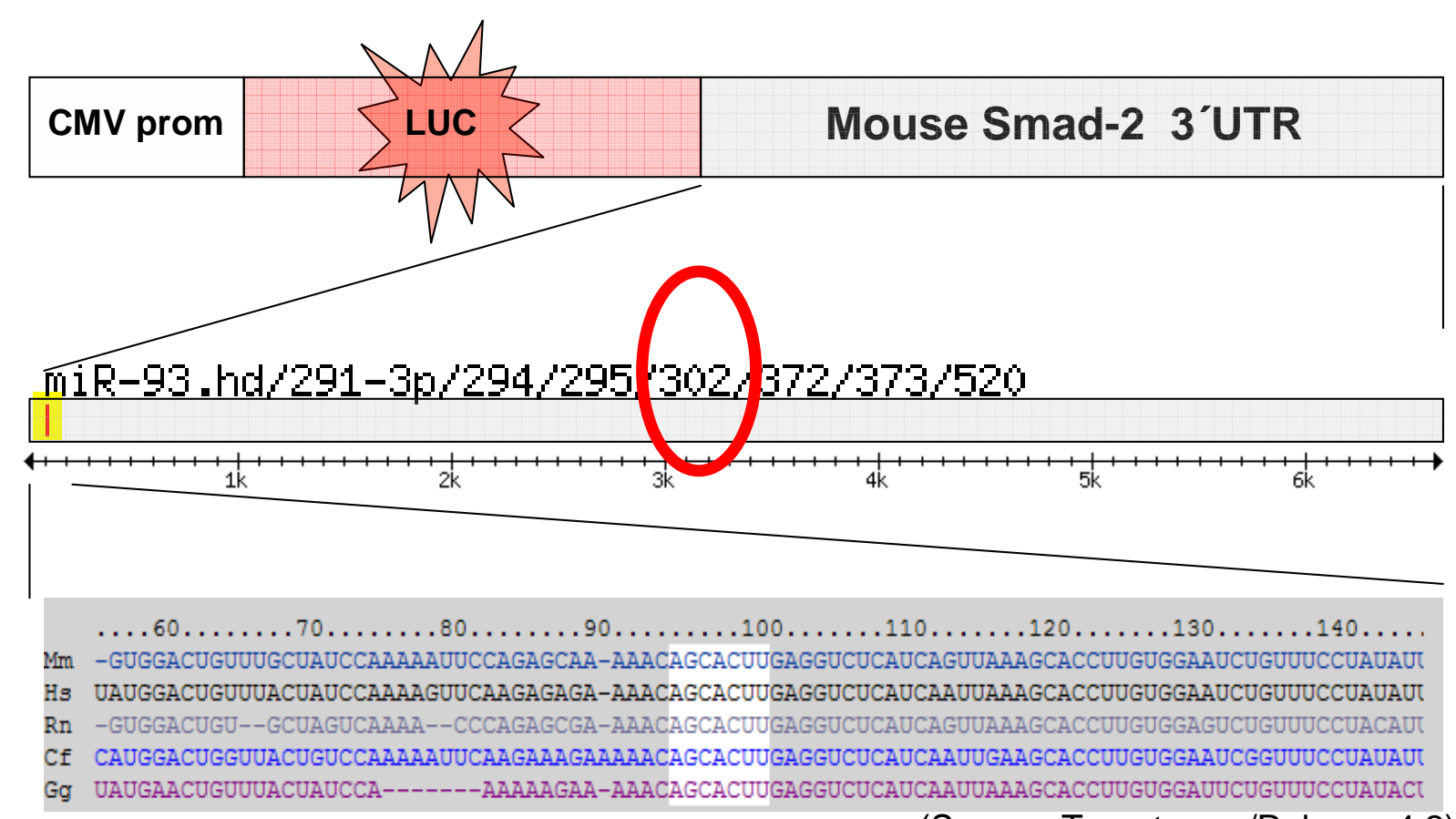

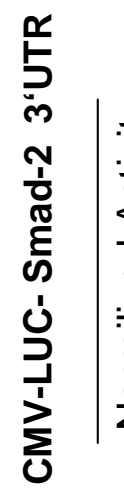

(Source: Targetscan /Release 4.2)

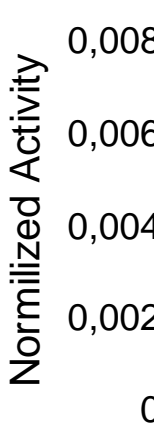

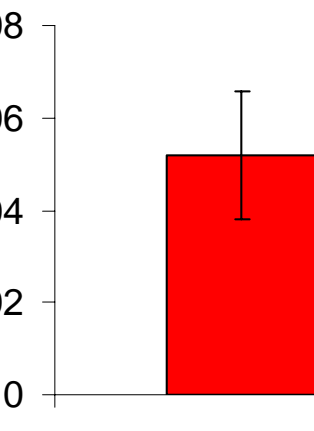

pos

neg

\section{Overexpression} miR-302 cluster

Figure 27. Luciferase assay for Smad2. A: The 3' UTR sequence for Smad2 was cloned in the Luciferase vector downstream of the Luciferase ORF. The 3' UTR of Smad2 contains sequences that, according to TargetScan (http://www.targetscan.org/vert_42/), are predicted as possible targets of, among other miRNAs, miR-302 cluster. B: Luciferase activity of NIH/3T3 cells cotransfected with the miRNA Luciferase Vector containing the 3' UTR sequence of Smad2 (CMV-LUC-Smad2 3' UTR) and the four pre-miRNAs of miR-302 cluster (pos) or the negative control (neg) was determined $24 \mathrm{hrs}$ after the transfection. Luciferase activity levels were normalized to the level of the $\beta$ galactosidase activity of the $\beta$-gal plasmid contransfected into the cells. Error bars indicate standard deviation. 


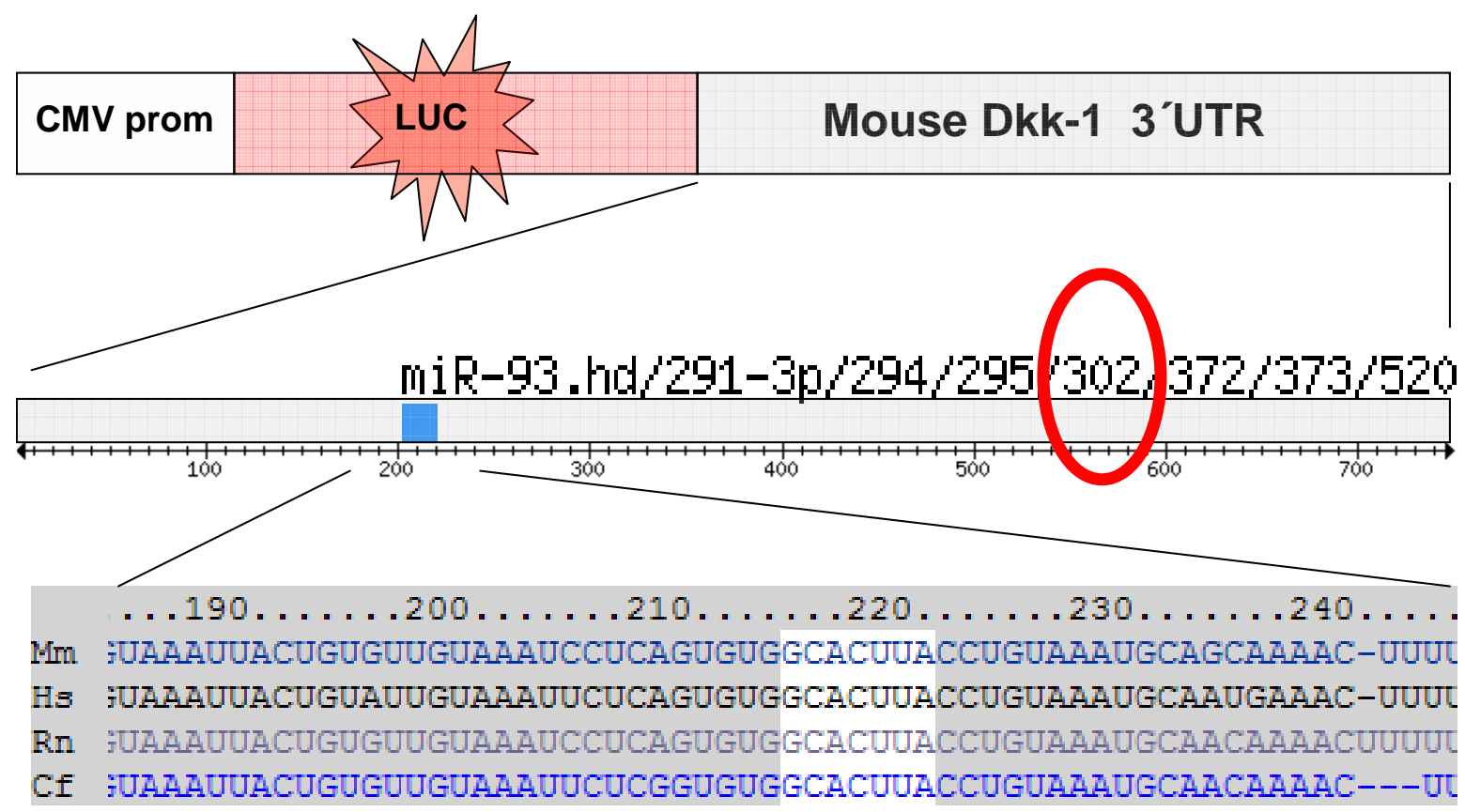

(Source: Targetscan /Release 4.2)

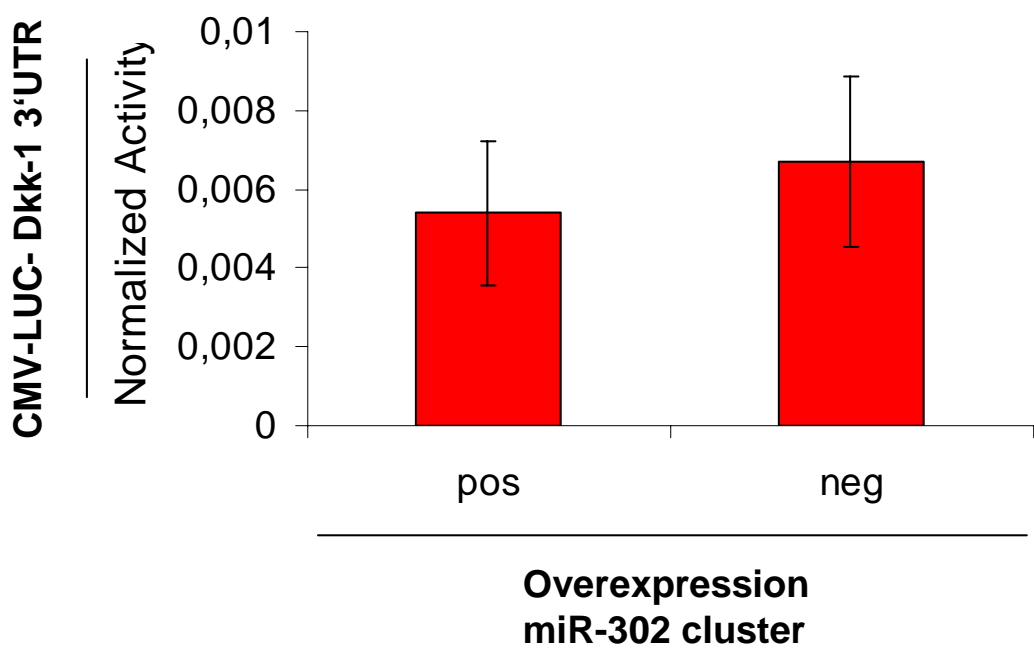

Figure 28. Luciferase assay for Dkk1. A: The 3' UTR sequence for Dkk1 was cloned in the Luciferase vector downstream of the Luciferase ORF. The 3' UTR of Dkk1 contains sequences that, according to TargetScan (http://www.targetscan.org/vert 42/), are predicted as possible targets of, among other miRNAs, miR-302 cluster. B: Luciferase activity of NIH/3T3 cells cotransfected with the miRNA Luciferase Vector containing the 3' UTR sequence of Dkk1 (CMV-LUC-Dkk1 3' UTR) and the four pre-miRNAs of miR-302 cluster (pos) or the negative control (neg) was determined $24 \mathrm{hrs}$ after the transfection. Luciferase activity levels were normalized to the level of the $\beta$ galactosidase activity of the $\beta$-gal plasmid contransfected into the cells. Error bars indicate standard deviation. 
Since Smad 2 and Dkk1 are, according to the Luciferase Assay, not targeted by miR-302 cluster, the reason why the expression of Brachyury and Dppa3 are inhibited by the overexpression of miR-302 cluster remains still unknown and, in consequence, the question why miR-302 cluster influences mesoderm and germ cell lineage formation could not be elucidated. 


\section{Discussion}

Differentiation of pluripotent stem cells is a challenging research field nowadays. On the one hand, stem cells that undergo differentiation can be used as research tools in order to elucidate the mechanisms of the early development. On the other hand, stem cells may hold the key of future transplantation research that will be able to replace and regenerate cells, tissues or even whole organs damaged during disease or injury (Kirschstein and Skirboll 2001).

miRNAs are a novel group of endogenous small RNAs whose function and role in post-transcriptional silencing of gene expression has attracted a growing scientific interest during the last few years. Among others, certain miRNAs are present exclusively in pluripotent stem cells and seem to influence their differentiation (Blakaj and Lin 2008).

This study concentrated on miRNAs whose levels are affected during differentiation of pluripotent cells. To this end, three pluripotent cell types were used, embryonic stem cells (ESCs), multipotent adult germ line stem cells (maGSCs) and embryonic germ cells (EGCs). ESCs and maGSCs were used in a global miRNA array screening, by which miRNAs that are downregulated after a 21-day differentiation can be detected.

In this study the global miRNA array profiling of ESCs and validation of the array data with qRT-PCR revealed two miRNA clusters whose levels decrease after differentiation: miR-17-92 and miR-290 clusters (see 3.1.1, p. 57, Table 1). The miRNA profile of maGSCs was identical with that of ESCs with the exception of miR-17-92 cluster, whose levels did not change after differentiation (see 3.1.2, p. 59, Table 2). For both ESCs and maGSCs levels of miR-302 cluster did not change during differentiation, although this miRNA cluster was 
described to be pluripotency-related. Since the effects of differentiation on miR290 and miR-302 clusters in ESCs and maGSCs have been already described (Zovoilis 2009), the present study examined in detail members of miR-17-92 cluster in the same cell types under various differentiation conditions. The results reinforced the array data, showing downregulation of these miRNAs in differentiating ESCs. In case of maGSCs tested members of miR-17-92 cluster remained in high levels (see 3.2, p. 67).

An additional pluripotent cell line, EGCs, was also investigated during differentiation (see 3.3, p. 76, Fig. 15) and its miRNA profile, although distinct, seems similar to this one previously described for maGSCs (Zovoilis et al. 2009).

In order to elucidate the role of miR-302 cluster in pluripotency and differentiation, overexpression of this cluster was conducted in ESCs, maGSCs and EGCs cultured under differentiation conditions. The overexpression of miR302 cluster failed to maintain the pluripotent state of the in vitro differentiated cells (see 3.4.1, p. 83, Fig. 19) but affected their differentiation, inhibiting formation of mesoderm and germ cell lineage (see 3.4.2.1, p. 86, Fig. 20, and 3.4.2.3, p. 91, Fig. 23). After investigation of Dkk1 and Smad2 genes, which participate in pathways controlling early mesoderm and germ cell lineage development, no possible target of miR-302 cluster could be identified (see 3.4.3.5, p. 103, Fig. 27 and p. 104, Fig. 28). 


\subsection{A miRNA overview}

miRNAs belong to the wide and diverse family of regulatory non-coding small RNAs. The first known miRNA, lin-4, was discovered in 1993 by Lee RC et al. in C.elegans, where it was found to repress the translation of lin-14, a controller of developmental timing (Lee RC et al. 1993). However, it was not until some years later that several publications revealed the presence of miRNAs in a range of eukaryotic organisms and established them as key players in RNA silencing (Lagos-Quintana et al. 2001; Lau et al. 2001; Lee RC and Ambros 2001).

The majority of miRNAs is transcribed in the nucleus as a part of a long primary form which is then processed gradually to hairpin-shaped precursors and finally to mature miRNAs (for detailed review of miRNA biogenesis see 1.4.1, p. 7). In this final form miRNAs contain a critical region of approximately 5-8 nt near their 5' end, which displays the highest level of complementarity (near perfect match) to the 3' UTR of targeted mRNAs. This miRNA region interacts with the mRNA, which leads to its degradation or translational repression according to the level of complementarity (Kim 2005).

miRNA-coding genes can be found in intronic regions, either in protein-coding or in non-coding sites, or in exonic regions of the genome (Barroso-del Jesus et al. 2009). One single miRNA can target several different mRNAs. Conversely, multiple binding of miRNAs on a mRNA is possible and induces a synergistic translational inhibition (Kim 2005).

According to miRBase Sequence Database, Release 12.0, a searchable online repository for published microRNA sequences in primates, rodents, birds, fish, worms, flies, plants and viruses (http://microrna.sanger.ac.uk/), 488 mouse and 695 human miRNAs are until now described. More than $30 \%$ of all human 
genes are thought to be miRNA targets (Bushati and Cohen 2007). However, with relatively few exceptions, we know little about the precise roles of the vast majority of miRNAs in regulating gene expression. Several studies have shown that miRNAs have diverse roles in numerous regulatory pathways, including early development, apoptosis and cell proliferation, carcinogenesis, differentiation and organ development (Esquela-Kerscher and Slack 2006; Kim 2005).

miRNAs have been implicated in a number of human diseases such as Fragile $\mathrm{X}$ syndrome, one of the most commonly inherited mental retardation syndromes. This syndrome is associated with loss of the Fragile X Mental Retardation 1 protein (FMR1) caused by a massive CGG trinucleotide repeat expansion within the 5' UTR of the FMR1 gene (Zarnescu et al. 2005; Qurashi et al. 2007). FMR1 protein belongs to a well conserved family of RNA-binding proteins and has been suggested to function as a translational repressor (Zarnescu et al. 2005). Current studies have investigated extensively the implication of Drosophila fragile X mental retardation ( $d F m r 1)$ gene in miRNA pathway. In 2002 it was demonstrated that dFmr1 protein associates with Argonaute2 (Ago2) protein and the RNA Induced Silencing Complex (RISC), which mediates miRNA translational repression (Caudy et al. 2002; Ishizuka et al. 2002). Later $d F m r 1$ was also shown to be associated with dAgo1 (Zarnescu et al. 2005). FMR protein was found to interact with miRNAs in both Drosophila and mammals (Jin et al. 2004). These observations strongly support the hypothesis that $d F m r 1$ protein might modulate translation of its mRNA targets by recruiting RISC along with miRNAs to them (Zarnescu et al. 2005).

miRNA expression profiles have been reported to be altered in sporadic cases of Alzheimer's disease (AD). Accumulation of amyloidal ß-peptides is known to play an important role in $\mathrm{AD}$ molecular pathogenesis. A protein named beta-site 
APP-cleaving enzyme 1 (BACE1) participates in the formation of amyloidal $\beta$ peptides. Increased BACE1 expression is observed in patients with sporadic AD (Hébert et al. 2007). Hébert et al. (2007) have demonstrated that members of $\mathrm{miR}-29 \mathrm{a} / \mathrm{b}-1$ cluster inhibit the expression of BACE1 protein. Expression of this miRNA cluster is significantly decreased in sporadic AD patients, resulting in abnormal overexpression of BACE1 gene.

In two patients suffering from Tourette's syndrome, a neuropsychiatric disorder characterized by vocal and motor tips, a correlation between miRNA function and the existing phenotype has been reported. In these patients a candidate gene for Tourette's syndrome, SLIT and NTRK-like family, member 1 (SLITRK1), contains a single-base mutation in its 3' UTR. The mutation is located in the mRNA sequence that is complement to miR-189 (this sequence serves presumably as a binding site of miR-189 to 3'UTR of SLITRK1 mRNA). This suggests a possible connection between inability of miR-189 to bind on SLITRK1 mRNA and, thus, to control its translation and abnormal expression of SLITRK1 gene with consequent development of Tourette's syndrome (Abelson et al. 2005).

Similarly, Beetz et al. (2008) reported two disease-associated variations in the 3' UTR of receptor accessory protein 1 gene (REEP1), which is a known cause of autosomal dominant Hereditary Spastic Paraplegia type SPG31. In two families a unique in each case single-nucleotide substitution was found within predicted conserved miRNA target sites. Alteration of these target sites led to aberrant REEP1 mRNA targeting and, therefore, to the disease phenotype.

The most extensive research regarding the role of miRNAs in human diseases concerns cancer, which is caused by uncontrolled proliferation and inappropriate survival of damaged cells. miRNAs have been proposed to contribute to 
oncogenesis, acting either as tumour suppressors or as oncogenes. The genomic abnormalities that influence miRNA function in tumours are similar to those known in protein-coding genes participating in cancer pathogenesis, such as genomic amplifications or deletions and point mutations (Calin and Croce 2006).

Several miRNAs seem to have a crucial function in cancer progression due to their location in genomic regions that are associated with cancer, known as fragile sites. For example, miR-125b-1, a homologue of lin-4 in C.elegans, was found to be located in a fragile site of human chromosome 11q24. This site and, thus, miR-125b-1 was shown to be deleted in some patients with breast, lung, ovarian and cervical cancer (Calin et al. 2002). Also miR-15a and miR-16-1 reside in $13 \mathrm{q} 14$ locus, which is found to be deleted in more than $65 \%$ of all Bcell chronic lymphocytic leukaemia (CLL) cases, in $60 \%$ of prostate cancer cases, in $16-40 \%$ multiple myelomas and in $50 \%$ of mantle cell lymphomas (Calin et al. 2002; Esquela-Kerscher and Slack 2006). miR-15a and miR-16-1 possibly regulate negatively BCL2, an anti-apoptotic gene often overexpressed in leukaemias and lymphomas (Cimmino et al. 2005).

miRNAs that could function as tumour suppressors in humans are miRNAs with proapoptotic activity, such as miRNAs of let-7 family. These miRNAs have been demonstrated to regulate negatively the RAS oncogenes in C.elegans and in human cancer cell lines (Johnson et al. 2005). Takamizawa et al. (2004) described a strong correlation between expression of members of the let- 7 family and post-operative survival in patients with lung cancer and suggest that let-7 could be used diagnostically, as patients with non-small-cell lung carcinomas who express low levels of let-7 have a poor prognosis. 
A miRNA cluster with antiapoptotic activity, miR-17-92 cluster, was shown to display oncogenic activity via several cancer pathways such as this of c-Myc gene and to be overexpressed in B cell lymphomas and in several solid tumours, such as breast, colon, lung, pancreas, prostate and stomach cancer (He et al. 2005; Cho 2007; Mendell 2008). It is believed that some of the members of miR-17-92 cluster function cooperatively as oncogenes, possibly by targeting apoptotic factors that are normally activated in response to c-Myc overexpression. By blocking the apoptotic pathways, c-Myc can induce uncontrolled cell proliferation that results in cancer (Esquela-Kerscher and Slack 2006).

\subsection{The role of miRNAs in pluripotent stem cells: miR-290 cluster}

The members of miR-290 cluster (miR-290, miR-291, miR-292, miR-293, miR294 and miR-295) are the most abundant miRNAs in ESCs and are silenced during early differentiation (Houbaviy et al. 2003, 2005). miR-290 cluster is also highly expressed in maGSCs (Zovoilis et al. 2008) and primordial germ cells (PGCs) (Hayashi et al. 2008), from which EGCs are derived.

The results of the global microRNA array expression profiling in ESCs and maGSCs in this study demonstrate a specific expression of miR-290 cluster in undifferentiated ESCs and maGSCs. After 21 days of differentiation in the presence of RA a strong downregulation of miR-290 cluster is observed (see 3.1.3.1, p. 61, Fig. 5). These data are consistent with previous findings in ESCs and maGSCs (Houbaviy et al. 2003; Zovoilis et al. 2008, Fig. 29). When the differentiation of ESCs and maGSCs is performed in gelatine condition but without RA, Zovoilis et al. showed that ESCs are early deprived of miR-290 cluster. However, according to their study, maGSCs sustain a comparable to undifferentiated cells expression of miR-290 cluster after differentiation. 


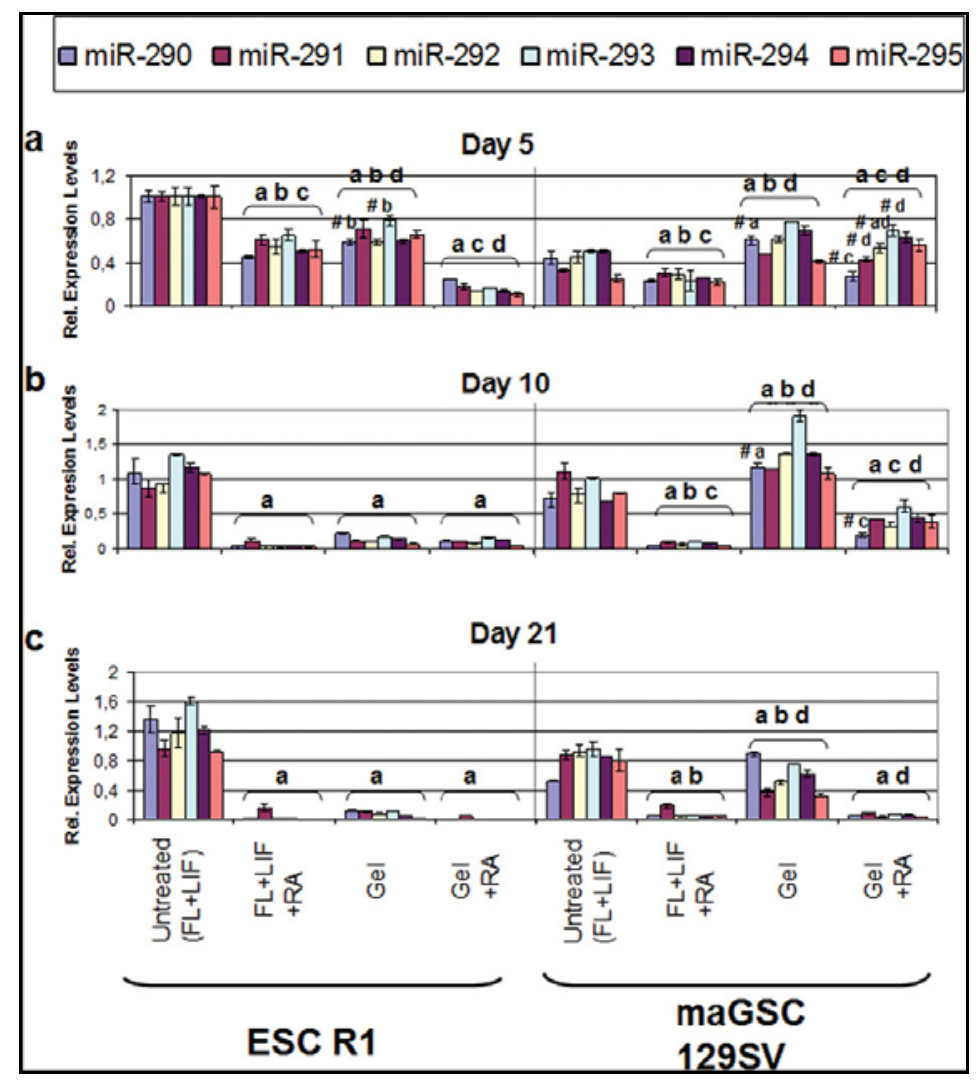

Figure 29. Expression of miR-290 cluster in ESCs and maGSCs during differentiation (kindly supplied from A.Zovoilis (Zovoilis et al. 2008)). Expression profiles of members of the miR-290 cluster in ESCs and maGSCs from mouse strain 129/Sv (ESC R1 and maGSC 129SV) under different culture conditions after 5, 10 and 21 days in culture (Fig. a, b and c, respectively).

Investigation of another pluripotent cell line, EGCs, exhibits also downregulation of miR-290 cluster when differentiation is performed in the presence of RA, as in the array experiment. However, when RA is not included in the differentiating conditions, EGCs display, as maGSCs, high levels of miR290 cluster during differentiation (see 3.3, p. 76, Fig. 15A). The importance of this finding concerning EGCs is discussed extensively in the next section (see 4.3 , p. 115). 
In order to elucidate the role of miR-290 cluster many studies have concentrated on knockout ESC models concerning important enzymes for the biogenesis of miRNAs, such as Dicer and Dgcr8. Loss of these enzymes is expected to result in complete miRNA depletion and this leads to abnormal differentiation or lacking proliferation. In detail, the loss of Dicer in mouse ESCs causes differentiation defects in vivo and in vitro (Kanellopoulou et al. 2005). This phenotype can be rescued by transfection with miR-290 cluster, which probably control DNA methylation via regulation of Rbl2 gene (Sinkkonen et al. 2008; Benetti et al. 2008). Moreover, Dgcr8-knockout ESCs proliferate slowly and accumulate in G1 phase of the cell cycle. This defective proliferation can be reversed after transfection with miR-290 cluster, demonstrating a central role of this cluster in G1-S transition and cell proliferation (Wang et al. 2008).

In consistence with the results of the above studies our group has shown that miR-290 cluster modulates the in vitro differentiation of ESCs by affecting Dkk1, an inhibitor of Wnt/ß-catenin pathway (Zovoilis et al. 2009). Wnt/ßcatenin pathway is known to play a central role in maintenance of pluripotency and incorrect activation leads to impaired differentiation (Nusse 2008). In mouse and human ESCs, activation of Wnt pathway was shown to be adequate in maintaining of self-renewal. Wnt signalling is endogenously activated in undifferentiated mouse ESCs and is downregulated upon differentiation (Sato et al. 2004). In addition, the ability of mouse ESCs to differentiate into the three germ layers is disturbed when incorrect activation of $\mathrm{Wnt} / \mathrm{B}$-catenin pathway occurs (Kielman et al. 2002).

Interestingly, miR-430, the zebrafish homologue of the mammalian miR-290 cluster, controls the expression of both a Nodal agonist (squint) and two Nodal antagonists (Lefty1 and Lefty2). Thus, miR-430 regulates by this fine tuning Nodal signalling pathway, which is important for early development (Choi et al. 
2007). This regulation of Nodal signalling by miR-430 is likely to be conserved across other species. Marson et al. (2008) revealed that key transcriptional factors in ESCs, such as Oct4, Sox2, Nanog and Tcf3, appear to promote the expression of Lefty1 and Lefty 2 and simultaneously activate miR-290 cluster that target the Lefty1 and Lefty2 3' UTRs.

From all the above mentioned findings it becomes apparent that miR-290 cluster is an important regulator of both self-renewal and differentiation in pluripotent stem cells.

\subsection{The role of miRNAs in pluripotent stem cells: miR-17-92 cluster}

miR-17-92 cluster encodes six members (miR-17, miR-18a, miR-19a, miR-20a, miR-19b and miR-92a). In addition, two miR-17-92 cluster paralogs exist, miR106b-25 and miR-106a-363 clusters (Mendell 2008). Several members of miR17-92 cluster and its paralogs have been shown to be highly expressed in undifferentiated ESCs (Suh et al. 2004, Tang et al. 2006).

As it has been already mentioned (see 4.1, p. 108), in humans miR-17-92 cluster seems to act as an oncogene, whose overexpression leads to various cancer types including both hematopoietic malignancies and solid tumours (Mendell 2008). Members of miR-17-92 cluster and its paralogs are shown to control ESC mesoderm and endoderm differentiation via targeting of STAT3, a transcription factor important for stem cell biology (Foshay and Gallicano 2009).

In this study the performed miRNA array profiling in ESCs and maGSCs (see 3.1.1, p. 57, Table 1, and 3.1.2, p. 59, Table 2) illustrated a striking and unique 
difference between these two pluripotent cell lines after differentiation: the expression of members of the miR-17-92 cluster is suppressed during differentiation in ESCs whereas the same miRNAs maintain their expression levels during differentiation of maGSCs. Apart from the exception of miR-17-92 cluster, differentiation miRNA profiles of ESCs and maGSCs are almost identical. Similarly to the miR-17 profile of maGSCs, in EGCs miR-17 also exhibits, according to the present data (see 3.3, p. 76, Fig. 15C), high expression during differentiation. EGCs are derived from primordial germ cells (PGCs), where it has been shown that miR-290 and miR-17-92 clusters are highly expressed and promote cell proliferation (Hayashi et al. 2008). This connection between EGCs and maGSCs concerning both miR-290 and miR-17-92 clusters could be attributed to their common origin from the germline and may suggest that maGSCs, despite their acquired pluripotent state, sustain to a certain degree germ cell features regarding their miRNA profile.

In the gelatine culture condition maGSCs maintain high expression levels of Oct4 longer than the differentiating ESCs (Zovoilis et al. 2008). Therefore, it can be assumed that maGSCs endure partially the differentiating pressure of the gelatine culture condition and differentiate slower than ESCs. It can be only speculated that this trait of maGSCs is connected with the persistent presence of miR-17-92 cluster during differentiation. Further functional studies could presumably shed light on the role of miR-17-92 cluster in maGSCs. 


\subsection{The role of miRNAs in pluripotent stem cells: miR-302 cluster}

The members of miR-302 cluster (miR-302a, miR-302b, miR-302c and miR302d) are highly expressed in undifferentiated cells, such as ESCs and maGSCs (Suh et al. 2004; Zovoilis et al. 2008).

In human ESCs the expression of miR-302a depends on the transcription factors Oct 4 and Sox 2 and miR-302a and miR-302b seem to regulate the cell cycle of ESCs and, thus, to exercise an important control over the "stemness" of these cells via targeting of Cyclin D1 and Cyclin D2, respectively (Card et al. 2008; Lee NS et al. 2008; Barroso-del Jesus et al. 2009). Moreover, ectopic expression of miR-302 cluster in human cancer lines was reported to be sufficient to reprogram them into pluripotent ESC-like cells, which express the ESC markers Oct4, SSEA3, SSEA4, Sox2 and Nanog, display a highly methylated genome similar to this of human ESCs and differentiate into several cell types (Lin et al. 2008).

As far as the role of miR-302 cluster in mouse pluripotent cells is concerned, few is known. Members of miR-302 cluster are induced during first stages of in vitro differentiation in mouse ESCs and maGSCs (Zovoilis et al. 2008). Global miRNA array profiling of ESCs and maGSCs shows no difference between undifferentiated and differentiated cells in the expression levels of miR-302 cluster. These data come in contradiction to the findings of Zovoilis et al. in the Gel+RA culture condition. Both ESCs and maGSCs display a significant downregulation of their miR-302 cluster expression levels after 21 days of differentiation (Fig. 30). 


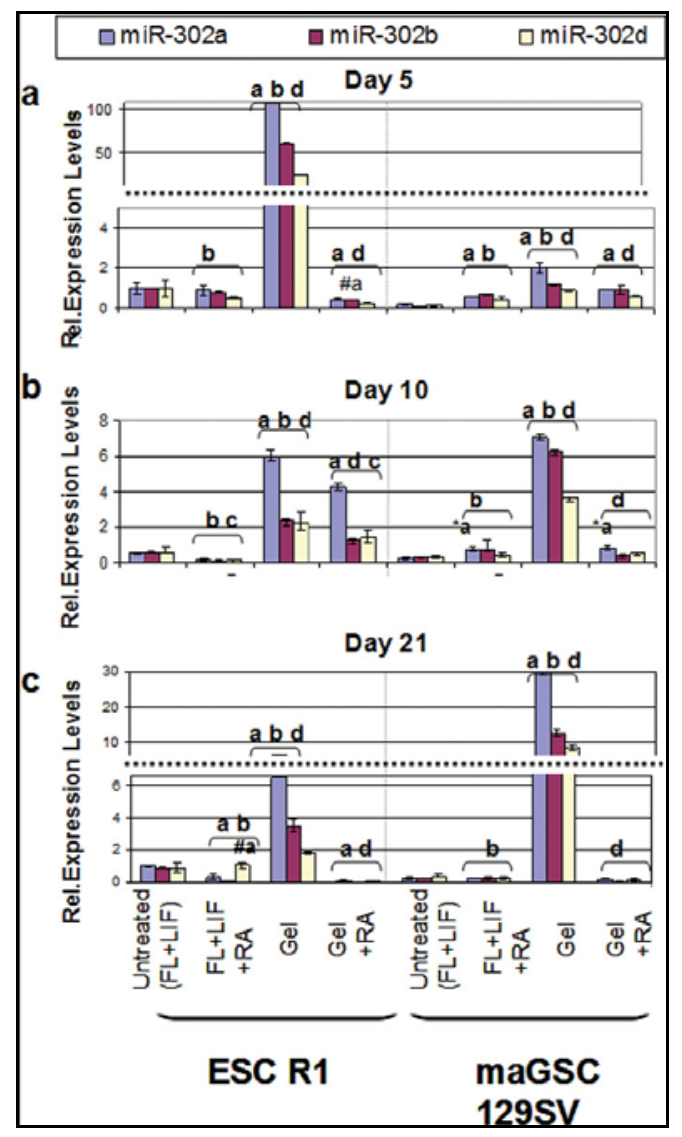

Figure 30. Expression of miR-302 cluster in ESCs and maGSCs during differentiation (kindly supplied from A.Zovoilis (Zovoilis et al. 2008)). Expression profiles of members of the miR-302 cluster in ESCs and maGSCs from mouse strain 129/Sv (ESC R1 and maGSC 129SV) under different culture conditions after 5, 10 and 21 days in culture (Fig. a, b and c, respectively).

As far as the gelatine condition is concerned, levels of miR-302 cluster in ESCs surprisingly increase during the first five days of differentiation (20-100fold upregulation) and later decrease but they still remain higher in comparison to undifferentiated cells. In maGSCs a gradual increase of miR-302 cluster is observed during differentiation in the gelatine condition (Fig. 28). The discrepancy between these findings and data that show a negative correlation between miR-302 cluster expression levels and differentiation over time is obvious (Houbaviy et al. 2003). Zovoilis et al. suggested that miR-302 cluster is more likely to be involved in the response mechanism of pluripotent stem cells 
to differentiation rather than in the maintenance of the undifferentiated state itself.

The differentiation of EGCs in the Gel+RA condition demonstrates an expression profile of miR-302a similar to that of the other tested pluripotent cell lines. However, in the Gel culture condition miR-302a displays an up- and downregulation expression pattern later and weaker (9-fold upregulation) in comparison to differentiating ESCs but earlier than in maGSCs (see 3.3, p. 76, Fig.15B).

In order to examine closer the influence of miR-302 cluster in mouse pluripotent stem cells the strategy of functional overexpression of the cluster in differentiating cells was chosen. Using this approach it could be demonstrated that high levels of miR-302 cluster were not able to prevent or delay loss of pluripotency, which was assessed by analysis of Oct4 expression levels (see 3.4.1, p. 83, Fig.19). These results indicate that, despite its potential association with pluripotency, miR-302 cluster alone is inadequate to maintain pluripotent state under differentiating culture conditions.

\section{5 miR-302 cluster is a key regulator of differentiation in pluripotent stem cells}

The role of miRNAs in differentiation of pluripotent stem cells has not yet been fully elucidated. Regarding ESC differentiation, Alisch et al. (2007) reported that loss of Argonaute2 (Ago2) results in gastrulation arrest and embryonic death, ectopic expression of the mesoderm marker Brachyury and mesoderm expansion. Mouse Ago2 protein participates in the RNA Induced Silencing Complex (RISC) and is closely associated with miRNA function, suggesting 
that Ago2 influences Brachyury expression and mesoderm development via the miRNA pathway (Alisch et al. 2007).

In the present study a detailed investigation of the influence of miR-302 cluster on in vitro differentiation of pluripotent stem cells could be achieved by functional overexpression of the cluster in differentiating cells. The use of a culture system containing serum directs the differentiation process towards mesoderm development (Bruce et al. 2007). Differentiation towards the mesoderm lineage was monitored by expression levels of mesoderm markers such as Brachyury, Eomesodermin and Fgf-8 (see 3.4.2.1, p. 86, Fig. 20 and p. 88, Fig. 21). The expression of these genes is inhibited due to high levels of miR-302 cluster, suggesting that this miRNA cluster prevents in vitro differentiation of ESCs and EGCs towards mesoderm. maGSCs demonstrate poor expression of mesoderm markers in undifferentiated and in differentiated state, therefore the influence of miR-302 cluster overexpression on the expression of mesoderm markers in differentiating maGSCs could not be estimated under current culture conditions.

Suppression of mesoderm formation does not favour neuroectoderm development, since differentiating ESCs submitted to miR-302 cluster overexpression and the respective control ESCs show no difference in expression of ectoderm marker Nestin (see 3.4.2.2, p. 90, Fig. 22).

Moreover, it was of great interest to examine whether miR-302 cluster affects, additionally to mesoderm formation, differentiation towards the germ cell lineage. Primordial germ cells (PGCs), the founders of germ cell lineage, originate from somatic cells of the proximal epiblast, which express mesoderm genes. During PGC specification expression of mesoderm markers is repressed while Dppa3 expression is upregulated, suggesting that Dppa3 play an important 
role in PGC specification and in their distinction from neighbouring somaticmesoderm cells (Tres et al. 2004; Hayashi et al. 2007). In tested ESCs and maGSCs overexpression of miR-302 cluster inhibited the expression of the germ cell marker Dppa3. However, due to the fact that differentiating EGCs show a strong downregulation of Dppa3 levels, the effects of miR-302 cluster overexpression on Dppa3 expression levels could not be evaluated in this differentiation culture system in the case of EGCs (see 3.4.2.3, p. 91, Fig. 23).

\subsection{Searching for targets of miR-302 cluster}

This study demonstrates that ectopic overexpression of miR-302 cluster modifies differentiation of pluripotent stem cells and alters the expression of mesoderm and germ cell lineage in differentiated cells. However, it is not yet clear which genes are targeted by miR-302 cluster.

According to computational algorithms, such as TargetScan, a widely used bioinformatics web tool, the 3' UTRs of Brachyury and Dppa3 mRNAs do not contain predicted sequences that could be recognised and targeted by members of miR-302 cluster (see 3.4.3.1, p. 93, Fig. 24). This search for computationally predicted targets of miR-302 cluster was then expanded to several genes that participate in Bmp4, Nodal and Wnt/ß-catenin signalling pathways, which have a pivotal role in formation of both mesoderm and germ cell lineage (Chen et al. 2004; Sudheer and Adjaye 2007; Tam and Loebel 2007). In total, 13 genes were tested using TargetScan. Five of them contained sequences that could be targeted by members of miR-302 cluster and were conserved across different species. These were Smad2, Dkk1, Tob2, Lefty1 and Lefty2 (see 3.4.3.1, p. 97, Table 3). 
In order to confirm experimentally these predicted targets of miR-302 cluster a Luciferase assay was used. Smad2 and Dkk1 were selected to be further tested. However, we could not show that the 3' UTRs of these two genes are affected by miR-302 cluster (see 3.4.3.5, p. 103, Fig. 27 and p. 104, Fig. 28). This could be attributed to the fact that false positive software predictions concerning miRNA targets are estimated at about $31 \%$ of all predicted targets in mouse (Lewis et al. 2003).

Present results do not exclude that the other predicted targets, Tob2, Lefty1 and Lefty2, could be confirmed as real targets of miR-302 cluster using the same Luciferase assay. Moreover, it can not be ruled out that other factors, which belong to Bmp4, Nodal and Wnt/ß-catenin pathways and were not examined in this study, may be targeted by this cluster and, thus, may play a key role in mesoderm and germ cell lineage formation. Therefore, investigation of further predicted miR-302 cluster targets with the use of Luciferase assay belong to the perspectives of the present study.

\subsection{Future work-perspectives}

The implication of miRNAs in pluripotency and early development is not fully clarified and many open questions and directions for future research remain. For example, impact of miR-302 cluster during differentiation could be also tested in loss-of function experiments. To this end, derivation of conditional knockout ESCs for miR-302 cluster would help to confirm the key role of this cluster in in vitro differentiation of ESCs that was observed here. In addition, these studies should be extended to in vivo models, such as the respective knockout mice. 
Until now, functional studies of our group have concentrated on miR-290 and miR-302 clusters. However, the role of miR-17-92 cluster in ESCs, EGCs and maGSCs remains unclear. The background of the results regarding the different expression profiles of miR-17-92 cluster in ESCs and in maGSCs remains still unclear. These data have to be interpreted in order to estimate the role of this miRNA cluster in proliferation and differentiation potential of maGSCs. Functional studies involving overexpression and inhibition of miR-17-92 cluster in these cells would serve this aim.

Finally, investigation of the role of miRNAs in differentiation of pluripotent stem cells should in the future contain also the newly characterized iPS cells (Takahashi and Yamanaka 2006; Wernig et al. 2007) and should also be extended in human pluripotent stem cells. 


\section{Summary}

One of the aims of this study was to provide a comprehensive view of the whole miRNAome of differentiating maGSCs (multipotent adult Germline Stem Cells) compared with that of differentiating ESCs (Embryonic Stem Cells) in mouse. To this end, whole miRNA array expression profiling of undifferentiated and differentiated ESCs and maGSCs (cultured unter differentiation conditions for 21 days) derived from a 129/Sv mouse background was performed. The array experiments revealed almost an identical miRNA expression profile between ESCs and maGSCs cultured under differentiation conditions. The only difference between differentiating ESCs and maGSCs concerned miR-17 and miR-20a. Expression levels of these two miRNAs did not decrease in maGSCs cultured under differentiation conditions, but they decreased in ESCs cultured under the same conditions. These results were confirmed with real-time qRTPCR. miR-17 and miR-20a belong to a group of miRNAs called oncomirs. This group includes the members of miR-17-92 cluster and its paralogs. Expression levels of representative members of miR-17-92 cluster (miR-17, miR-19b, miR20a, miR-20b) were then tested with qRT-PCR in ESCs and maGSCs that were cultured under differentiation conditions for 5, 10 and 21 days. The obtained results concerning the tested members of miR-17-92 cluster confirmed the data from the array experiments. These differentiation studies were extended to an additional pluripotent cell line, EGCs (Embryonic Germ Cells). Regarding expression of members of miR-290 and miR-17-92 clusters during differentiation, EGCs have important similarities with maGSCs. In order to investigate in detail the role of miR-302 cluster in pluripotency and differentiation of pluripotent stem cells, all four members of the cluster were overexpressed in differentiating ESCs, maGSCs and EGCs. This in vitro approach revealed that miR-302 cluster, although designated as pluripotencyrelated, can not impede the differentiation process itself. However, this cluster 
alters the character that differentiating cells obtain and high levels of its members disrupt expression of markers of mesoderm (Brachyury, Eomesodermin, Fgf-8) and germ cell lineage (Dppa3) and, therefore, hinder proper differentiation towards these two cell lineages. Using TargetScan Software several possible targets of miR-302 cluster were identified. These predicted targets participate in Bmp4, Nodal and Wnt/ß-catenin signaling pathways, which are important for mesoderm and germ cell lineage formation. Two of these possible targets, Smad2 and Dkk1, were experimentally examined and were shown not to be affected by miR-302 cluster. 


\section{References}

Abelson JF, Kwan KY, O’Roak BJ, Baek DY, Stillman AA, Morgan TM, Mathews CA, Pauls DL, Rašin M, Gunel M et al. (2005): Sequence variants in SLITRK1 are associated with Tourette's syndrome. Science $\underline{310}$, 317-320

Alisch RS, Jin P, Epstein M, Caspary T, Warren ST (2007): Argonaute2 is essential for mammalian gastrulation and proper mesoderm formation. PLoS Genetics $\underline{3}$, e227

Ambros V (2004): The functions of animal microRNAs. Nature 431, 350-355

Ambrosi DJ, Tanasijevic B, Kaur A, Obergfell C, O’Neill RJ, Krueger W, Rasmussen TP (2007): Genome-wide reprogramming in hybrids of somatic cells and embryonic stem cells. Stem Cells $\underline{25}, 1104-1113$

Barroso-del Jesus A, Lucena-Aguilar G, Menendez P (2009): The miR-302-367 cluster as a potential stemness regulator in ESCs. Cell Cycle $\underline{8}, 394-398$

Bartel DP (2004): MicroRNAs: Genomics, biogenesis, mechanism, and function. Cell 116, 281-297

Bartel DP, Chen CZ (2004): Micromanagers of gene expression: the potentially widespread influence of metazoan microRNAs. Nat Rev Genet $\underline{5}, 396-400$

Beetz C, Schüle R, Deconinck T, Tran-Viet KN, Zhu H, Kremer BP, Frints SG, van Zelst-Stams WA, Byrne P, Otto S, Nygren AO et al. (2008): REEP1 mutation spectrum and genotype/phenotype correlation in hereditary spastic paraplegia type 31 . Brain $\underline{131}, 1078-1086$ 
Benetti R, Gonzalo S, Jaco I, Munoz P, Gonzalez S, Schoeftner S, Murchison E, Andl T, Chen T, Klatt P et al. (2008): A mammalian microRNA cluster controls DNA methylation and telomere recombination via Rbl2-dependent regulation of DNA methyltranferases. Nat Struct Mol Biol 15, 268-279

Blakaj A, Lin H (2008): Piecing together the mosaic of early mammalian development through microRNAs. J Biol Chem 293, 9505-9508

Brons IG, Smithers LE, Trotter MW, Rugg-Gunn P, Sun B, Chuva de Sousa Lopes SM, Howlett SK, Clarkson A, Ahrlund-Richter L, Pedersen RA et al. (2007): Derivation of pluripotent epiblast stem cells from mammalian embryos. Nature $\underline{448}, 191-195$

Bruce SJ, Gardiner BB, Burke LJ, Gongora MM, Grimmond SM, Perkins AC (2007): Dynamic transcription programs during ES cell differentiation towards mesoderm in serum versus serum-free BMP4 culture. BMC Genomics $\underline{8}, 365$

Buitrago W, Roop DR (2007): Oct-4: The almighty POUripotent regulator? J Invest Derm 127, 260-262

Bushati N, Cohen SM(2007): microRNAs in neurodegenereration. Curr Opin Neurobiol $\underline{18}, 292-296$

Calin GA, Croce CM (2006): MicroRNA signatures in human cancers. Nat Rev Cancer $\underline{6}, 857-866$

Calin GA, Dumitru CD, Shimizu M, Bichi R, Zupo S, Noch E, Aldler H, Rattan S, Keating M, Rai K et al. (2002): Frequent deletions and down-regulation of 
micro- RNA genes miR15 and miR16 at 13q14 in chronic lymphocytic leukemia. Proc Natl Acad Sci USA 99, 15524-15529

Card DA, Hebbar PB, Li L, Trotter KW, Komatsu Y, Mishina Y, Archer TK (2008): Oct4/Sox2-regulated miR-302 targets Cyclin D1 in human embryonic stem cells. Mol Cell Biol 28, 6426-6438

Caudy AA, Myers M, Hannon GJ, Hammond SM (2002): Fragile X-related protein and VIG associate with the RNA interference machinery. Genes Dev $\underline{16}$, 2491-2496

Chen D, Zhao M, Mundy GR (2004): Bone morphogenetic proteins. Growth Factors $\underline{22}, 233-241$

Cho WC (2007): OncomiRs: the discovery and progress of microRNAs in cancer. Mol Cancer $\underline{6}, 60$

Choi W, Giraldez AJ, Schier AF (2007): Target protectors reveal dampening and balancing of Nodal agonist and antagonist by miR-430. Science $\underline{318}, 271-$ 274

Chung Y, Klimanskaya I, Becker S, Marh J, Lu SJ, Johnson J, Meisner L, Lanza R (2006): Embryonic and extraembryonic stem cell lines derived from single mouse blastomeres. Nature $\underline{439}, 216-219$

Cimmino A, Calin GA, Fabbri M, Iorio MV, Ferracin M, Shimizu M, Wojcik SE, Aqeilan RI, Zupo S, Dono M et al. (2005): mir-15 and mir-16 induce apoptosis by targeting BCL2. Proc Natl Acad Sci USA 102, 13944-13949 
Duparc RH, Boutemmine D, Champagne MP, Tétreault N, Bernier G (2006): Pax6 is required for delta-catenin/neurojugin expression during retinal, cerebellar and cortical development in mice. Dev Biol $\underline{300}$, 647-655

Esquela-Kerscher A, Slack FJ (2006): Oncomirs-microRNAs with a role in cancer. Nat Rev Cancer $\underline{6}$, 259-269

Evans MJ, Kaufman MH (1981): Establishment in culture of pluripotential cells from mouse embryos. Nature 292, 154-156

Foshay KM, Gallicano GI (2009): miR-17 family miRNAs are expressed during early mammalian development and regulate stem cell differentiation. Dev Biol $\underline{326}, 431-443$

Guan K, Nayernia K, Maier LS, Wagner S, Dressel R, Lee JH, Nolte J, Wolf F, Li M, Engel W et al. (2006): Pluripotency of spermatogonial stem cells from adult mouse testis. Nature $\underline{440}, 1199-1203$

Han DW, Do JT, Gentile L, Stehling M, Lee HT, Schöler HR (2008): Pluripotential reprogramming of the somatic genome in hybrid cells occurs with the first cell cycle. Stem Cells $\underline{26}, 445-454$

Han J, Pedersen JS, Kwon SC, Belair CD, Kim YK, Yeom KH, Yang WY, Haussler D, Blelloch R, Kim VN (2009): Posttranscriptional crossregulation between Drosha and DGCR8. Cell 136, 75-84

Hayashi K, Chuva de Sousa Lopes SM, Surani MA (2007). Germ cell specification in mice. Science 20 , 394-396 
Hayashi K, Chuva de Sousa Lopes SM, Kaneda M, Tang F, Hajkova P, Lao K, O Carroll D, Das PP, Tarakhovsky A, Miska EA et al. (2008): MicroRNA biogenesis is required for mouse primordial germ cell development and spermatogenesis. PLoS ONE $\underline{3}$, e1738

He L, Thomson JM, Hemann MT, Hernando-Monge E, Mu D, Goodson S, Powers S, Cordon-Cardo C, Lowe SW, Hannon GJ et al. (2005): A microRNA polycistron as a potential human oncogene. Nature $\underline{435}, 828-833$

Hébert SS, Horré K, Nicolai L, Papadopoulou AS, Mandemakers W, Silahtaroglu AN, Kauppinen S, Delacourte A, De Strooper B (2007): Loss of microRNA cluster miR-29a/b-1 in sporadic Alzheimer's disease correlates with increased BACE1/ß-secretase expression. Proc Natl Acad Sci USA 105, 64156420

Houbaviy H, Murray M, Sharp P (2003): Embryonic stem cell-specific microRNAs. Dev Cell $\underline{5}, 351-358$

Houbaviy H, Dennis L, Jaenisch R, Sharp PA (2005): Characterization of a highly variable eutherian microRNA gene. RNA $\underline{11}, 1245-1257$

http://microrna.sanger.ac.uk/ (2008)

http://www.exiqon.com/array (2008)

http://www.targetscan.org/vert_42/ (2008) 
Ishizuka A, Siomi MC, Siomi H (2002): A Drosophila fragile X protein interacts with components of RNAi and ribosomal proteins. Genes Dev $\underline{16}$, 2497-2508

Izadyar F, Pau F, Marh J, Slepko N, Wang T, Gonzalez R, Ramos T, Howerton K, Sayre C, Silva F (2008): Generation of multipotent cell lines from a distinct population of male germ line stem cells. Reproduction $\underline{135}, 771-784$

Jin P, Zarnescu DC, Ceman S, Nakamoto M, Mowrey J, Jorgens TA, Nelson DL, Moses K, Warren ST (2004): Biochemical and genetic interaction between the fragile $\mathrm{X}$ mental retardation protein and the microRNA pathway. Nat Neurosci $\underline{7}, 113-117$

Johnson SM, Grosshans H, Shingara J, Byrom M, Jarvis R, Cheng A, Labourier E, Reinert KL, Brown D, Slack FJ (2005): RAS is regulated by the let-7 microRNA family. Cell $\underline{120}, 635-647$

Kahan BW, Ephrussi B (1970): Developmental potentialities of clonal in vitro cultures of mouse testicular teratoma. J Natl Cancer Inst $\underline{44}$, 1015-1036

Kanatsu-Shinohara M, Inoue K, Lee J, Yoshimoto M, Ogonuki N, Miki H, Baba S, Kato T, Kazuki Y, Toyokuni S et al. (2004): Generation of pluripotent stem cells from neonatal mouse testis. Cell $\underline{119}, 1001-1012$

Kanellopoulou C, Muljo SA, Kung AL, Ganesan S, Drapkin R, Jenuwein T, Livingston DM, Rajewsky K (2005): Dicer-deficient mouse embryonic stem cells are defective in differentiation and centromeric silencing. Genes Dev $\underline{19}$, 489-510 
Keller G (2005): Embryonic stem cell differentiation: emergence of a new era in biology and medicine. Genes Dev $\underline{19}, 1129-1155$

Keller G, Kennedy M, Papayannopoulou T, Wiles MV (1993): Hematopoietic commitment during embryonic stem cell differentiation in culture. Mol Cell Biol $\underline{13}, 473-486$

Kielman MF, Rindapää M, Gaspar C, van Poppel N, Breukel C, van Leeuwen S, Taketo MM, Roberts S, Smits R, Fodde R (2002): Apc modulates embryonic stem-cell differentiation by controlling the dosage of beta-catenin signaling. Nat Genet $\underline{32}, 594-605$

Kim VN (2005): MicroRNA biogenesis: coordinated cropping and dicing. Nat Rev Mol Cell Biol $\underline{6}$, 376-385

Kirschstein R, Skirboll LR: Stem cells: Scientific progress and future research directions; in: NIH stem cells, National Institute of Health, Bethesda, Maryland/USA 2001, 15-18

Kleinsmith LJ, Pierce GB Jr. (1964): Multipotentiality of single embryonal carcinoma cells. Cancer Res 24, 1544-1551

Lagos-Quintana M, Rauhut R, Lendeckel W, Tuschl T (2001): Identification of novel genes coding for small expressed RNAs. Science 294, 853-858

Lau NC, Lim LP, Weistein EG, Bartel DP (2001): An abundant class of tiny RNAs with probable regulatory roles in Caenorhabditis elegans. Science 294, $858-862$ 
Lee NS, Kim JS, Cho WJ, Lee MR, Steiner R, Gompers A, Ling D, Zhang J, Strom P, Behlke M et al. (2008): miR-302b maintains "stemness" of human embryonal carcinoma cells by post-transcriptional regulation of Cyclin D2 expression. Biochem Biophys Res Commun 377, 434-440

Lee RC, Feinbaum RL, Ambros V (1993): The C. elegans heterochronic gene lin-4 encodes small RNAs with antisense complementarity to lin-14. Cell $\underline{75}$, $843-854$

Lee RC, Ambros V (2001): An extensive class of small RNAs in Caenorhabditis elegans. Science $\underline{294}, 797-799$

Lendahl U, Zimmerman LB, McKay RD (1990): CNS stem cells express a new class of intermediate filament protein. Cell $\underline{60}, 585-595$

Lengner C, Jaenisch R: Pluripotent cell isolation for regenerative medicine (2008). Nat Rev Mol Cell Biol, http://www.stemcell.com/technical/nrm0908poster-web.pdf

Lewis B, Shih I, Jones-Rhoades M, Bartel D, Burge C (2003): Prediction of mammalian microRNA targets. Cell 115, 787-798

Lin SL, Chang DC, Chang-Lin S, Lin CH, Wu DTS, Chen DT, Ying SY (2008): Mir-302 reprograms human skin cancer cells into a pluripotent ES-cell-like state. RNA $\underline{14}, 2115-2124$

Liu N, Lu M, Tian X, Han Z (2007): Molecular mechanisms involved in selfrenewal and pluripotency of embryonic stem cells. J Cell Physiol 211, 279-286 
Marson A, Levine SS, Cole MF, Frampton GM, Brambrink T, Johnstone S, Guenther MG, Johnston WK, Wernig M, Newman J et al. (2008): Connecting microRNA genes to the core transcriptional regulatory circuitry of embryonic stem cells. Cell 134, 521-533

Martin GR (1981): Isolation of a pluripotent cell line from early mouse embryos cultured in medium conditioned by teratocarcinoma stem cells. Proc Natl Acad Sci $\underline{78}, 7634-7638$

Matsui Y, Zsebo K, Hogan BLM (1992): Derivation of pluripotential embryonic stem cells from murine primordial germ cells in culture. Cell $\underline{70}, 841-847$

Mendell JT (2008): miRiad roles for the miR-17-92 cluster in development and disease. Cell 133, 217-222

Niwa H (2007): How is pluripotency determined and maintained? Development $\underline{134}, 635-646$

Nusse R (2008): Wnt signaling and stem cell control. Cell Res $\underline{18}$, 523-527

Qurashi A, Chang S, Jin P (2007): Role of microRNA pathway in mental retardation. Scientific WorldJournal ㄱ, 146-154

Resnick JL, Bixler LS, Cheng L, Donovan PJ (1992): Long-term proliferation of mouse primordial germ cells in culture. Nature $\underline{359}, 550-551$

Rolletschek A, Chang H, Guan K, Czyz J, Meyer M, Wobus AM (2002): Differentiation of embryonic stem cell-derived dopaminergic neurons is enhanced by survival-promoting factors. Mech Dev 105, 93-104 
Rubin DC (2007): Intestinal morphogenesis. Curr Opin Gastroenterol 23, 111114

Sanger F, Coulson AR (1975): A rapid method for determining sequences in DNA by primed synthesis with DNA polymerase. J Mol Biol 94, 441-446

Sato N, Meijer L, Skaltsounis L, Greengard P, Brivanlou AH (2004): Maintenance of pluriponcy in human and mouse embryonic stem cells through activation of Wnt signaling by a pharmacological GSK-3-specific inhibitor. Nat Med 10, 55-63

Schöler HR: The Potential of Stem Cells: An Inventory; in: Humanbiotechnology as Social Challenge: an interdisciplinary introduction to bioethics; edited by Knoepffler N, Schipanski D, Sorgner SL; Ashgate Publishing, Ltd., Surrey/UK 2007, 27-29

Seandel M, James D, Shmelkov SV, Falciatori I, Kim J, Chavala S, Scherr DS, Zhang F, Torres R, Gale NW et al. (2007): Generation of functional multipotent adult stem cells from GPR125+ germline progenitors. Nature $\underline{449}, 346-350$

Serov O, Matveeva N, Kuznetsov S, Kaftanovskaya E, Mittmann J (2001): Embryonic hybrid cells: a powerful tool for studying pluripotency and reprogramming of the differentiated cell chromosomes. An Acad Bras Cienc 73, $561-568$

Singh SK, Bhadra MP, Girschick HJ, Bhadra U (2008): MicroRNAs - micro in size but macro in function. FEBS J $\underline{275}$, 4929-4944

Sinkkonen L, Hugenschmidt T, Berninger P, Gaidatzis D, Mohn F, Artus-Revel CG, Zavolan M, Svoboda P, Filipowicz W (2008): MicroRNAs control de novo 
DNA methylation through regulation of transcriptional repressors in mouse embryonic stem cells. Nat Struct Mol Biol 15, 259-267

Sladek FM, Zhong WM, Lai E, Darnell JE (1990): Liver-enriched transcription factor HNF-4 is a novel member of the steroid hormone receptor superfamily. Genes Dev $\underline{4}, 2353-2365$

Stevens LC (1970): The development of transplantable teratocarcinomas from intratesticular grafts of pre- and postimplantation mouse embryos. Dev Biol 21, $364-382$

Stewart CL, Gadi I, Bhatt H (1994): Stem cells from primordial germ cells can reenter the germ line. Dev Biol 161, 626-628

Strauss W, Chen C, Lee CT, Ridzon D (2006): Nonrestrictive developemental regulation of microRNA gene expression. Mamm Genome 17, 833-840

Sudheer S, Adjaye J (2007): Functional genomics of human pre-implantation development. Brief Funct Genomic Proteomic $\underline{6}$, 120-132

Suh MR, Lee Y, Kim JY, Kim SK, Moon SH, Lee JY, Cha KY, Chung HM, Yoon HS, Moon SY et al. (2004): Human embryonic stem cells express a unique set of microRNAs. Dev Biol 270, 488-498

Takahashi K, Yamanaka S (2006): Induction of pluripotent stem cells from mouse embryonic and adult fibroblast cultures by defined factors. Cell $\underline{126}, 663-$ 676

Takamizawa J, Konishi H, Yanagisawa K, Tomida S, Osada H, Endoh H, Harano T, Yatabe Y, Nagino M, Nimura Y et al. (2004): Reduced expression of 
the let-7 microRNAs in human lung cancers in association with shortened postoperative survival. Cancer Res $\underline{64}$, 3753-3756

Tam PPL, Loebel DAF (2007): Gene function in mouse embryogenesis: get set for gastrulation. Nat Rev Genet $\underline{8}, 368-381$

Tang F, Hajkova P, Barton SC, Lao K, Surani MA (2006): MicroRNA expression profiling of single whole embryonic stem cells. Nucleic Acids Res $\underline{34}$, e9

Todaro GJ, Green H (1965): Successive transformations of an established cell line by Polyoma virus and SV40. Science 147, 513-514

Tres LL, Rosselot C, Kierszenbaum AL (2004): Primordial germ cells: what does it take to be alive? Mol Reprod Dev $\underline{68}, 1-4$

Wakayama S, Hikichi T, Suetsugu R, Sakaide Y, Bui HT, Mizutani E, Wakayama T (2007): Efficient establishment of mouse embryonic stem cell lines from single blastomeres and polar bodies. Stem Cells $\underline{25}, 986-993$

Wang Y, Baskerville S, Shenoy A, Babiartz JE, Baehner L, Blelloch R (2008): Embryonic stem cell-specific microRNAs regulate the G1-S transition and promote rapid proliferation. Nat Genet $\underline{40}, 1478-1483$

Wernig M, Meissner A, Foreman R, Brambrink T, Ku M, Hochedlinger K, Bernstein BE, Jaenisch R (2007): In vitro reprogramming of fibroblasts into a pluripotent ES-cell-like state. Nature $\underline{448}, 553-560$ 
Wiles MV, Johansson BM (1999): Embryonic stem cell development in a chemically defined medium. Exp Cell Res 247, 241-248

Wiles MV, Proetzel G: Controlling the differentiation of mouse ES cells in vitro; in: Embryonic Stem Cells: a practical approach; edited by Notarianni E, Evans MJ; Oxford University Press, New York/USA 2006, 112-128

Wurst W, Joyner AL: Production of targeted embryonic stem cell clones; in: Gene targeting: a practical approach; edited by Joyner AL; IRL Press, Oxford/UK 1993, 33-61

Ying QL, Nichols J, Chambers I, Smith A (2003): BMP induction of Id proteins suppresses differentiation and sustains embryonic stem cell self-renewal in collaboration with STAT3. Cell 115, 247-248

Yu J, Thomson JA (2008): Pluripotent stem cell lines. Genes Dev 22, 1987-1997

Zarnescu DC, Shan G, Warren ST, Jin P (2005): Come FLY with us: toward understanding fragile X syndrome. Genes Brain Behav $\underline{4}$, 385-392

Zovoilis A: MicroRNA expression profiling of multipotent adult germline stem cells Med. Diss. Göttingen 2009

Zovoilis A, Nolte J, Drusenheimer N, Zechner U, Hada H, Guan K, Hasenfuss G, Nayernia K, Engel W (2008): Multipotent adult germline stem cells and embryonic stem cells have similar microRNA profiles. Mol Hum Reprod 14, $521-529$ 
Zovoilis A, Smorag L, Pantazi A, Engel W (2009): Members of the miR-290 cluster modulate in vitro differentiation of mouse embryonic stem cells. (Differentiation, submitted) 


\section{Acknowledgments}

I would like to express my gratitude to Prof. Wolfgang Engel, Director of the Institute of Human Genetics of the University of Goettingen and leader of our research group. His expertise, understanding and patience added considerably to my graduate experience. I appreciate his vast knowledge and skill in the area of stem cell research and his assistance in writing this thesis. Prof. Engel has been a true professor/teacher to me and without his support I doubt that this work would have been achieved.

I also owe a lot to my supervisor and colleague, Dr. Athanasios Zovoilis, without whose motivation and encouragement I would not have considered my involvement in the research field of miRNAs and stem cells. It was under his tutelage that I developed a focus and became interested in miRNAs. He shared with me his valuable experience in cell culture and taught me the methods for evaluating miRNAs that were used in this study and which he had personally established in the Institute of Human Genetics. Under his guidance I was able to develop a "scientific" way of thinking and to evolve as a person.

A very special thanks goes out to Mr. Lukasz Smorag, with whom I have worked side by side during the last year and who supplied me with the Luciferase vectors used in the functional studies.

I must also acknowledge Mrs. Nadja Drusenheimer for her inestimable assistance and provision of materials concerning the Luciferase assay. Appreciation also goes out to for all collaborators of the Institute of Human Genetics for their assistance and comments throughout my research; I would like to thank all former and present members of AG Engel who have supported me in this work, especially Mrs. Sandra Meyer, Dr. Katayoon Shirneshan and 
Mrs. Janine Ulrich, as well as Mr. Krishna Pantakani and Mrs. Aleksandra Kata. Thanks also go out to Prof. Ibrahim Adham, Dr. Ashraf Mannan and Dr. Pawel Grzmil for their comments and advice, which lead to important improvements in this study.

Finally, I owe a debt of gratitude to Dr. G. Salinas and Mr. L. Opitz of the Array Core Facility of the University of Goettingen for the overall help that they provided with the conduction of the miRNA array experiments. 


\section{Curriculum Vitae}

I was born in Amarousio Attikis, Greece, on $18^{\text {th }}$ February 1982. In 1999 I graduated from the $1^{\text {st }}$ High School of Amarousio, Greece, with the note 19,3/20. From October 2000 until January 2007 I studied medicine in the Medical Faculty of the University of Athens, Greece, from where I graduated with the note "very good". I was a trainee medical student in the Department of Pediatrics of University Hospital of Sofia, Bulgaria, on August 2003 and in the Department of Internal Medicine of University Hospital of Geneva, Switzerland, on June 2005. From December 2005 until June 2006 I was a PJ student in the University Hospital of Cologne, Germany. From August until November 2007 I worked as a junior doctor in the Department of Internal Medicine (Cardiology) in Regional Hospital of Eschwege, Germany. Since January 2008 I have been a doctoral student in the Institute of Human Genetics of the University of Goettingen, where I have also started my residency in Medical Genetics. Apart from Greek, which is my native tongue, I can also speak fluently English, German and French. 